\title{
Structure and Dynamics of Crystalline Molecular Gyrotops with a Difluorophenylene Rotor
}

\author{
Taro Tsuchiya,${ }^{\dagger}$ Yusuke Inagaki, ${ }^{\dagger}$ Kentaro Yamaguchi, ${ }^{\ddagger}$ and Wataru Setaka ${ }^{\dagger},{ }^{\star}$ \\ †Division of Applied Chemistry, Faculty of Urban Environmental Sciences, Tokyo Metropolitan \\ University, 1-1 Minami-Osawa, Hachioji, Tokyo 192-0397, Japan \\ ‡ Faculty of Pharmaceutical Sciences at Kagawa Campus, Tokushima Bunri University, 1314-1 \\ Shido, Sanuki, Kagawa 769-2193, Japan
}

1. Copies of ${ }^{1} \mathrm{H},{ }^{13} \mathrm{C},{ }^{29} \mathrm{Si},{ }^{19} \mathrm{~F}$ NMR, and HRMS Spectra for All New Compounds

2. Details of X-Ray Diffraction Studies

3. Details of Temperature Dependent ${ }^{2} \mathrm{H}$ NMR Spectra

4. Details of Temperature Dependent Dielectric Relaxation Studies

5. Details of DFT Calculations 
1. Copies of ${ }^{1} \mathrm{H},{ }^{13} \mathrm{C} \quad$ NMR Spectra for All New Compounds
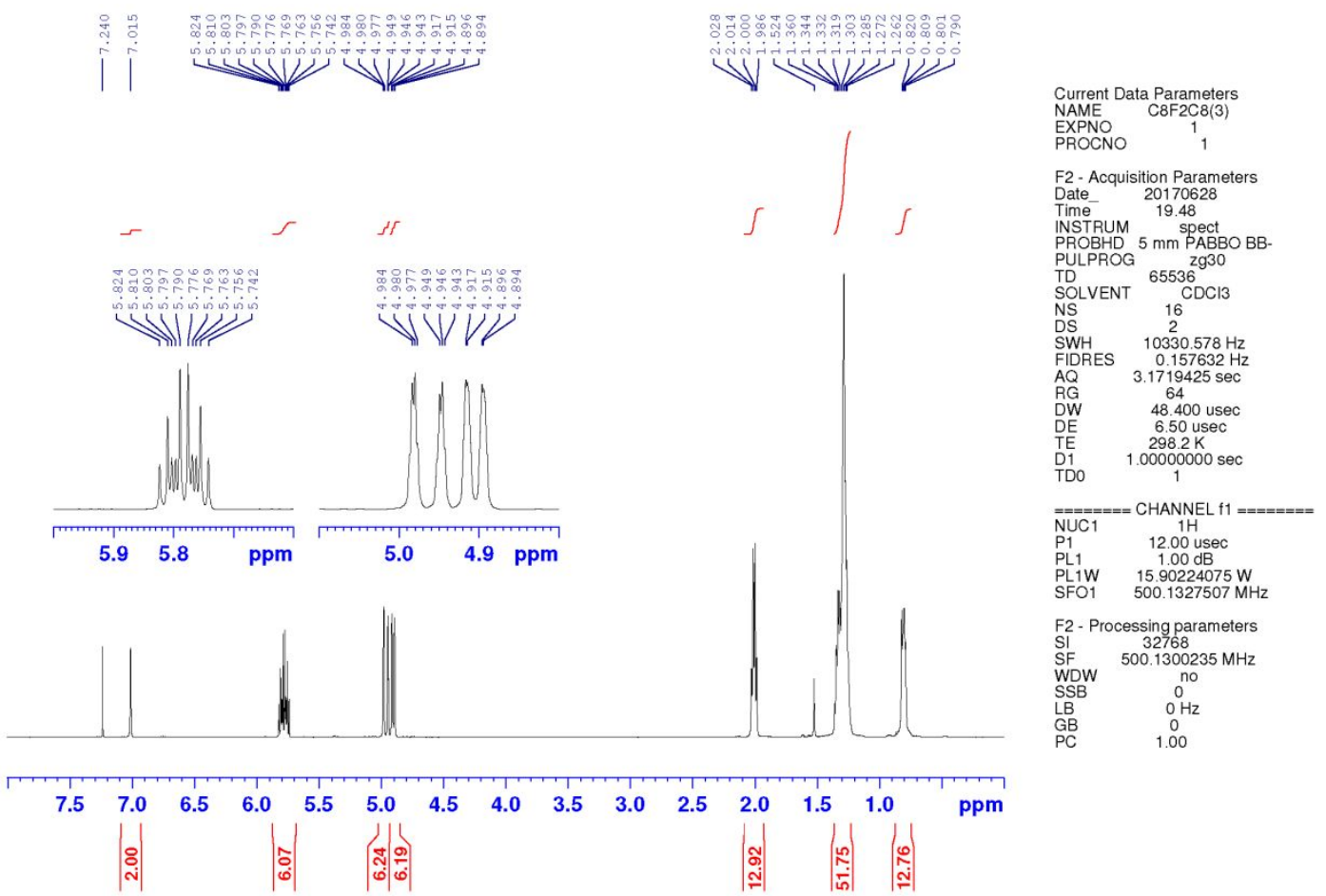

Figure S1. ${ }^{1} \mathrm{H}$ NMR (500 MHz) spectrum of 1,4-bis(tris-7-octenylsilyl)-2,3-difluorobenzene (1) in $\mathrm{CDCl}_{3}$.
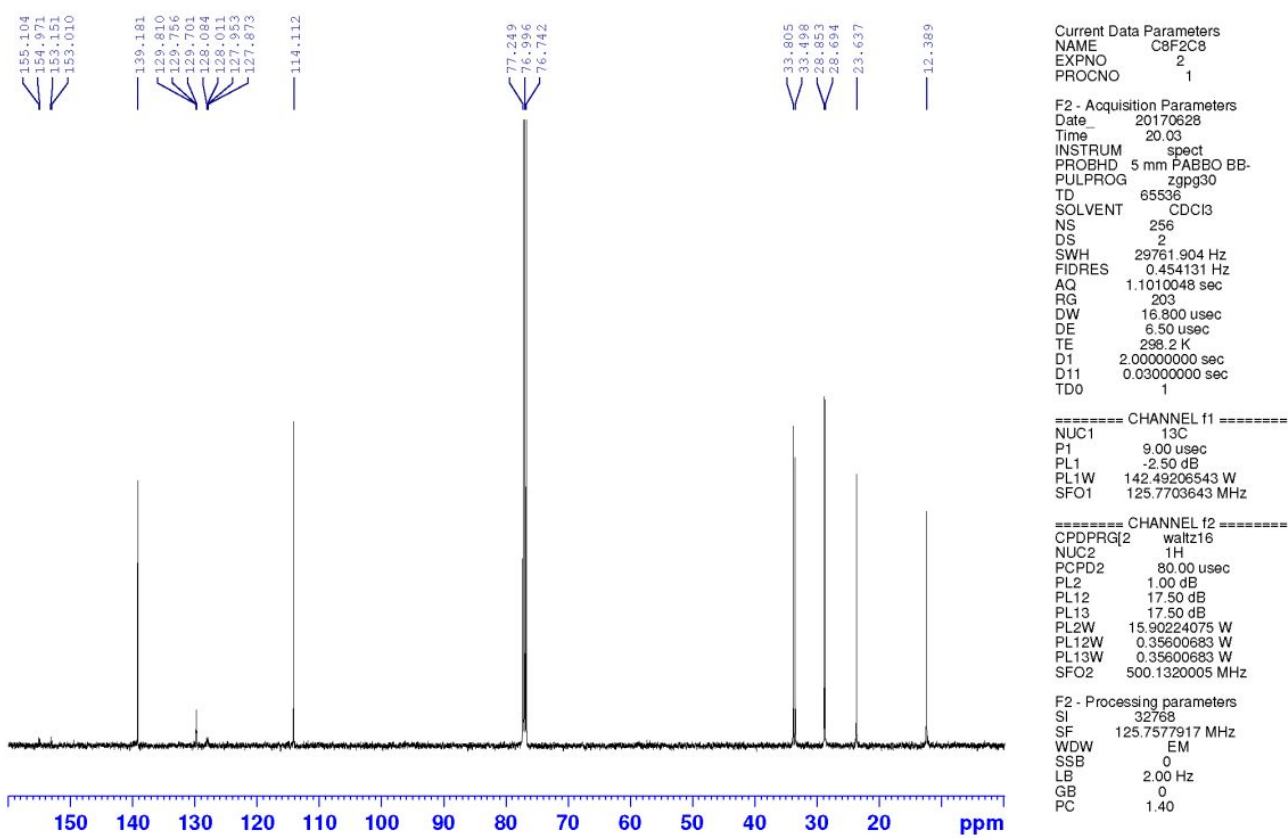

Figure S2. ${ }^{13} \mathrm{C}$ NMR (126 MHz) spectrum of 1,4-bis(tris-7-octenylsilyl)-2,3-difluorobenzene (1) in $\mathrm{CDCl}_{3}$. 


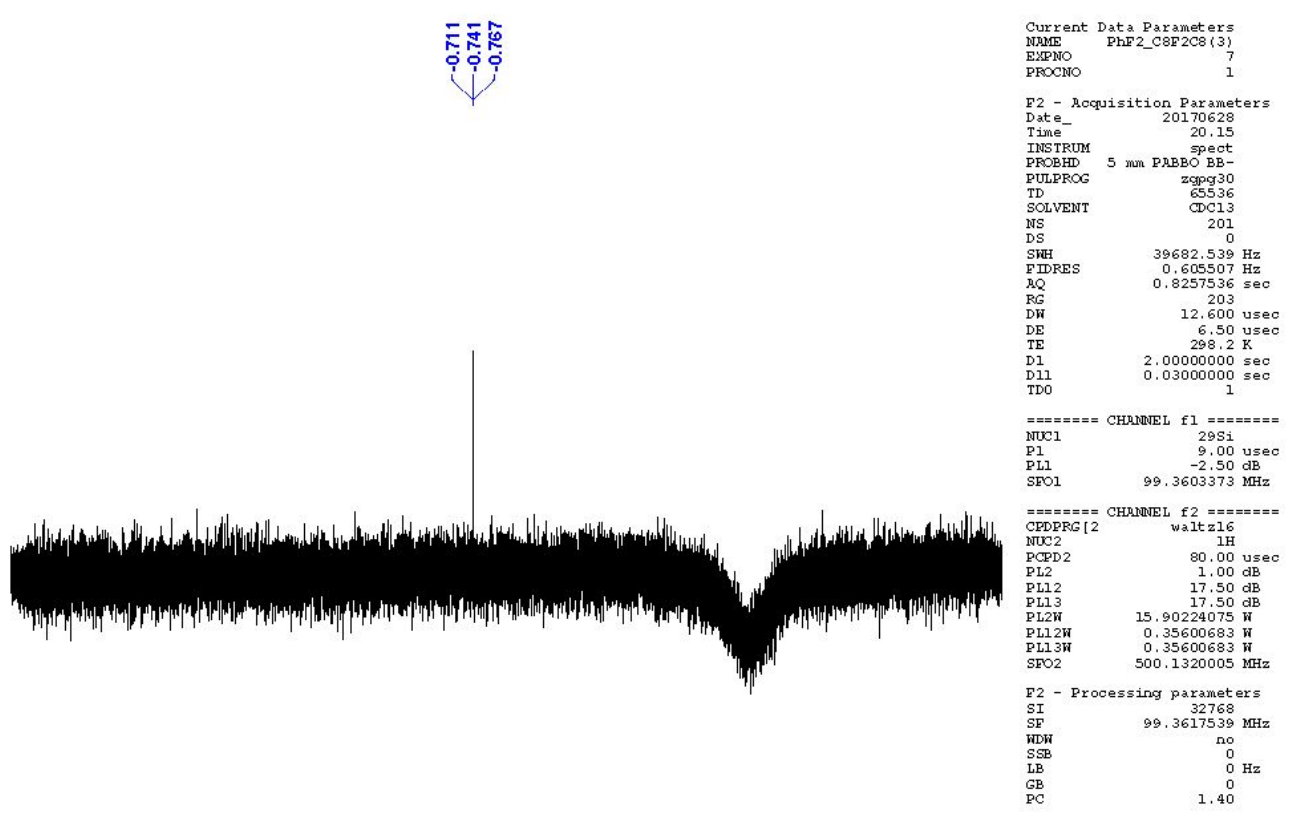

Figure S3. ${ }^{29} \mathrm{Si}$ NMR (99 MHz) spectrum of 1,4-bis(tris-7-octenylsilyl)-2,3-difluorobenzene (1) in $\mathrm{CDCl}_{3}$.

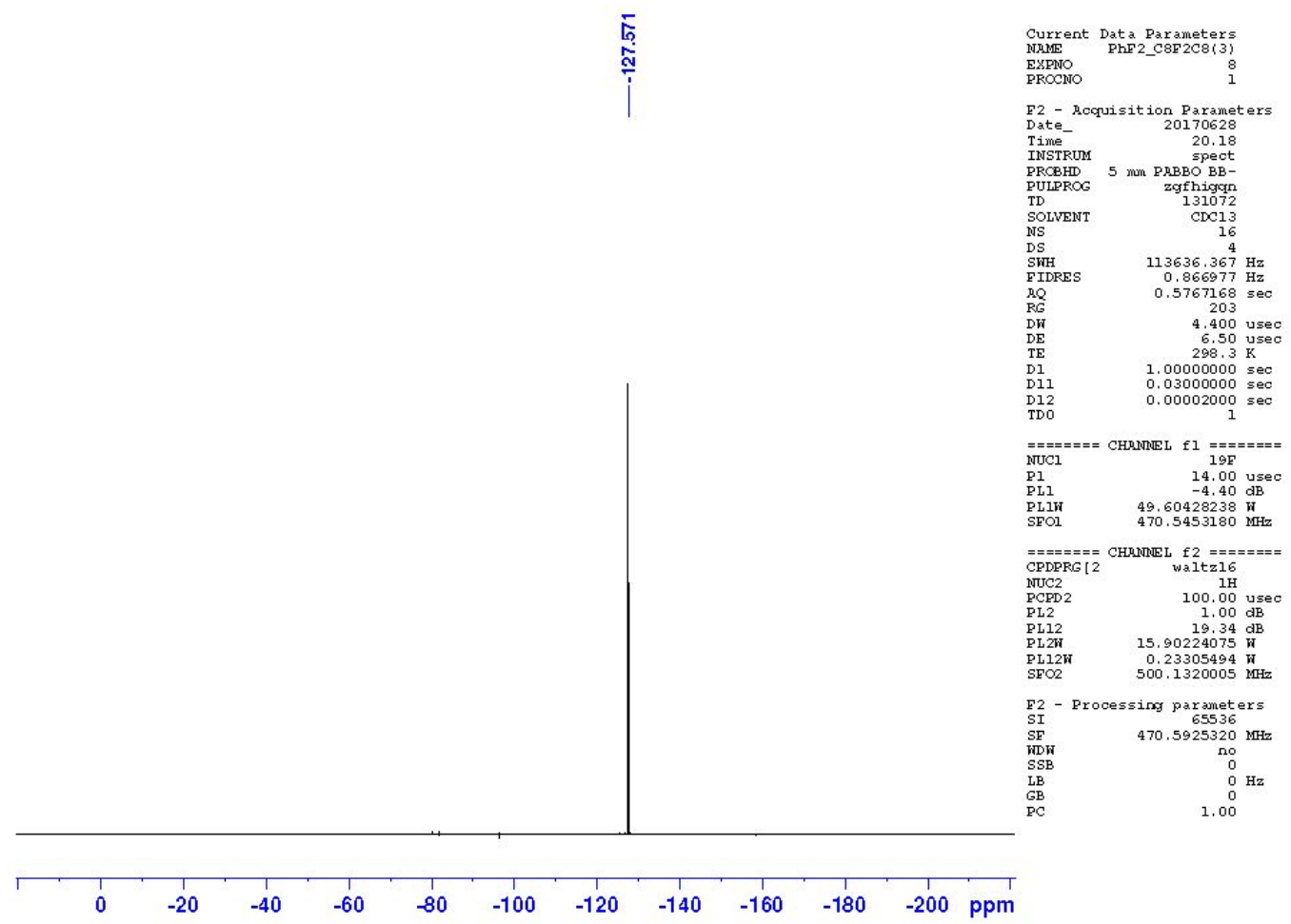

Figure S4. ${ }^{19} \mathrm{~F}$ NMR (471 MHz) spectrum of 1,4-bis(tris-7-octenylsilyl)-2,3-difluorobenzene (1) in $\mathrm{CDCl}_{3}$. 


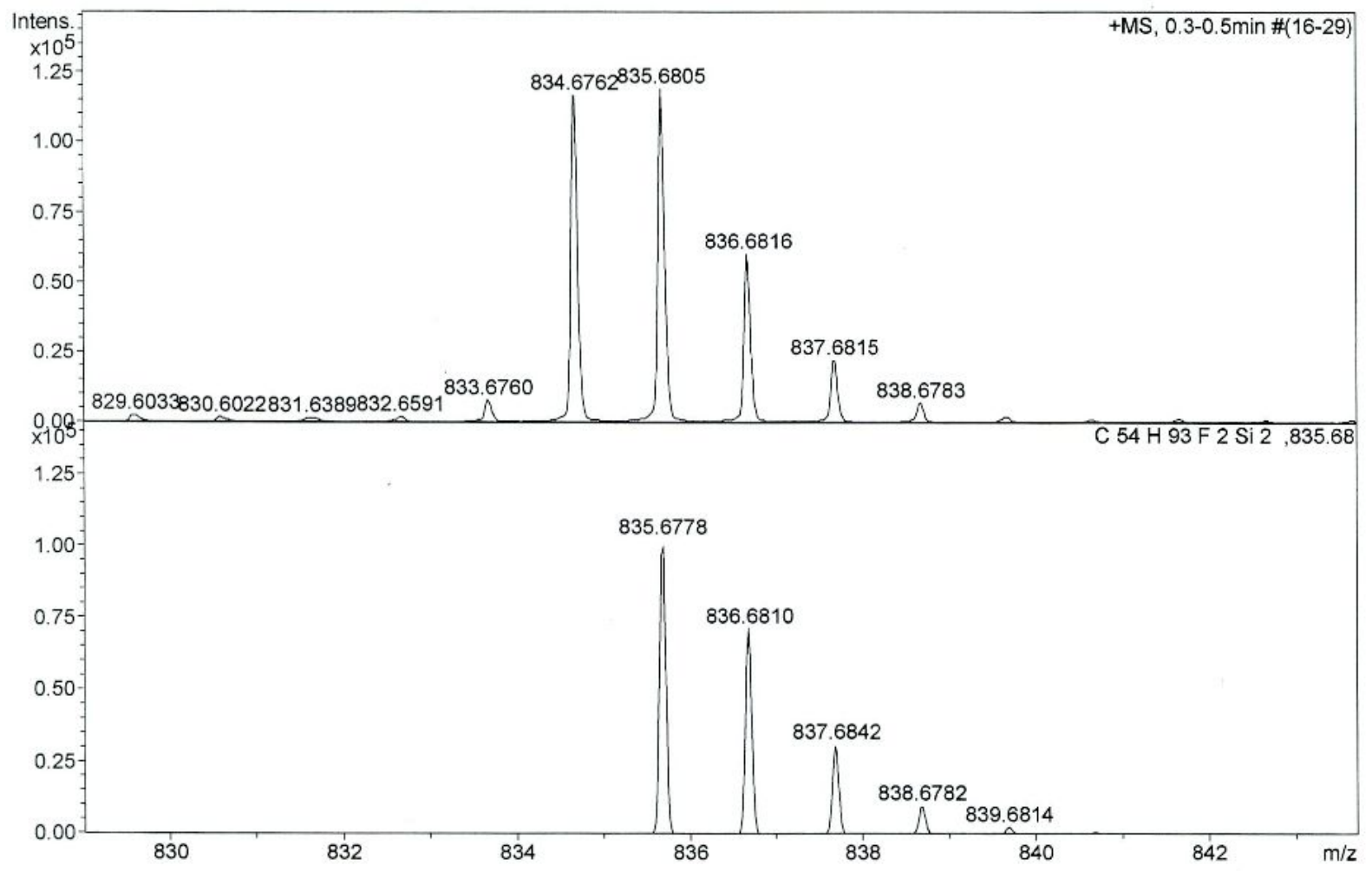

Figure S5. HRMS spectrum of 1,4-bis(tris-7-octenylsilyl)-2,3-difluorobenzene (1) (ESI, positive). Top: obsd. Bottom: sim.

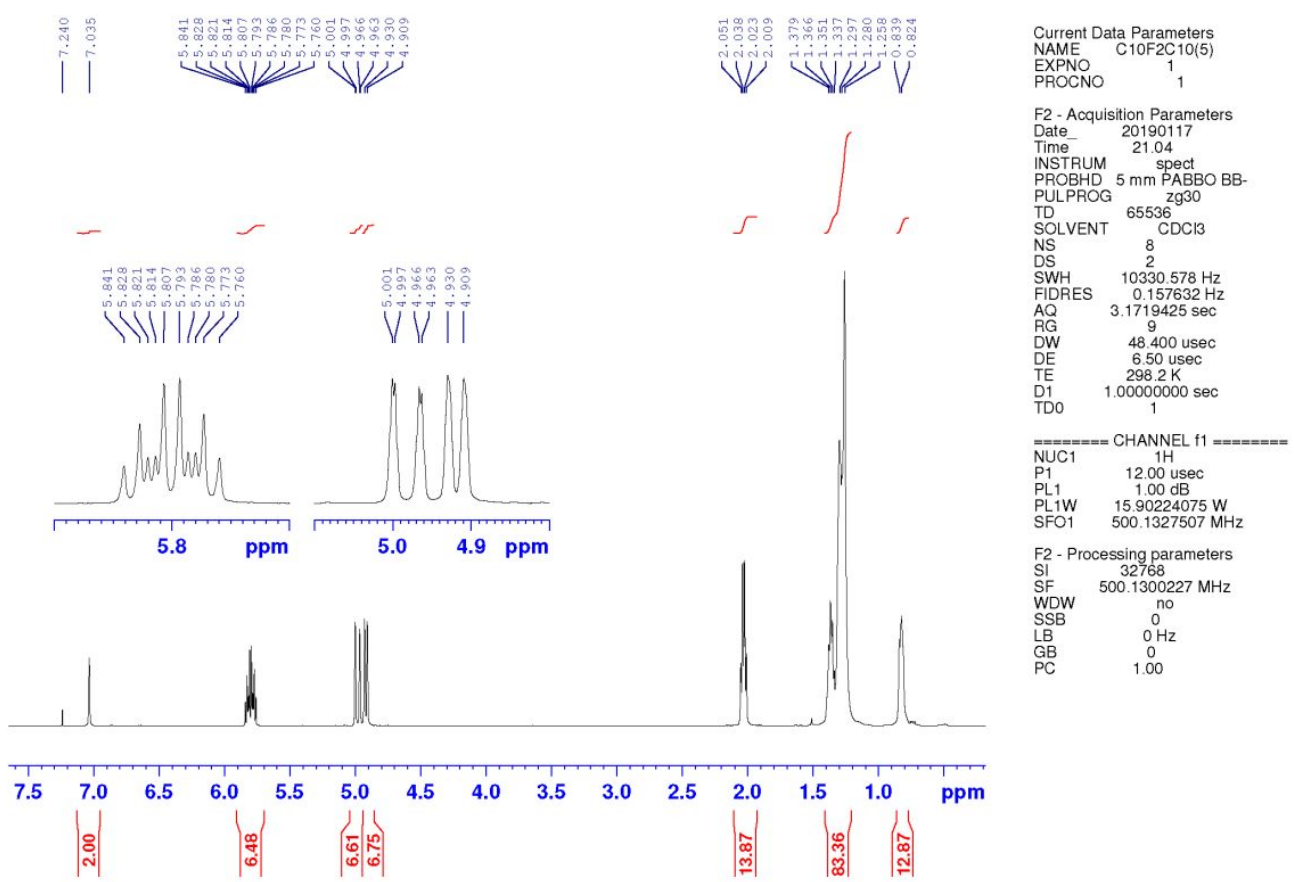

Figure S6. ${ }^{1} \mathrm{H}$ NMR (500 MHz) spectrum of 1,4-bis(tris-9-decenylsilyl)-2,3-difluorobenzene (2) in $\mathrm{CDCl}_{3}$. 


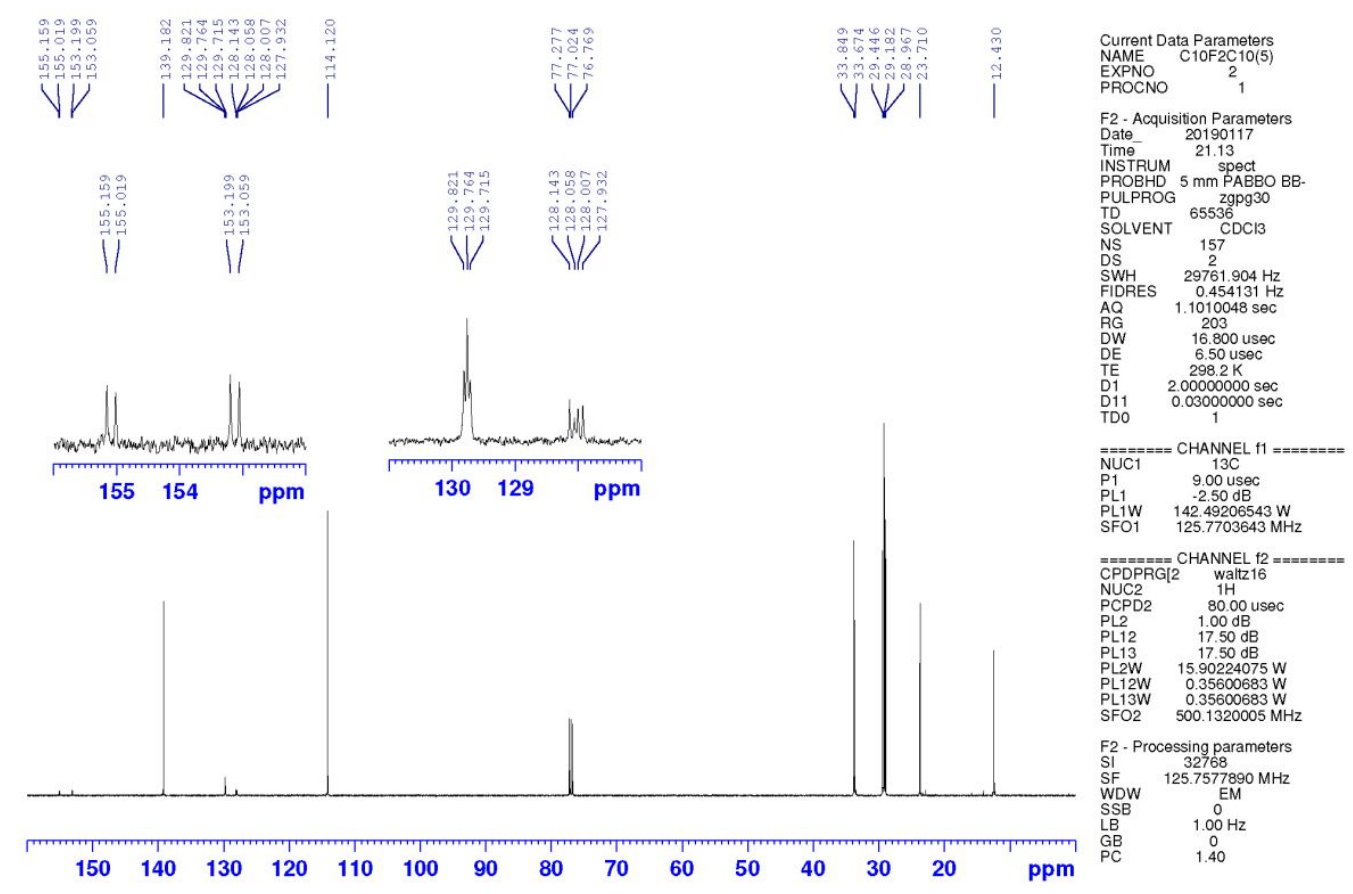

Figure S7. ${ }^{13} \mathrm{C}$ NMR (126 MHz) spectrum of 1,4-bis(tris-9-decenylsilyl)-2,3-difluorobenzene (2) in $\mathrm{CDCl}_{3}$.

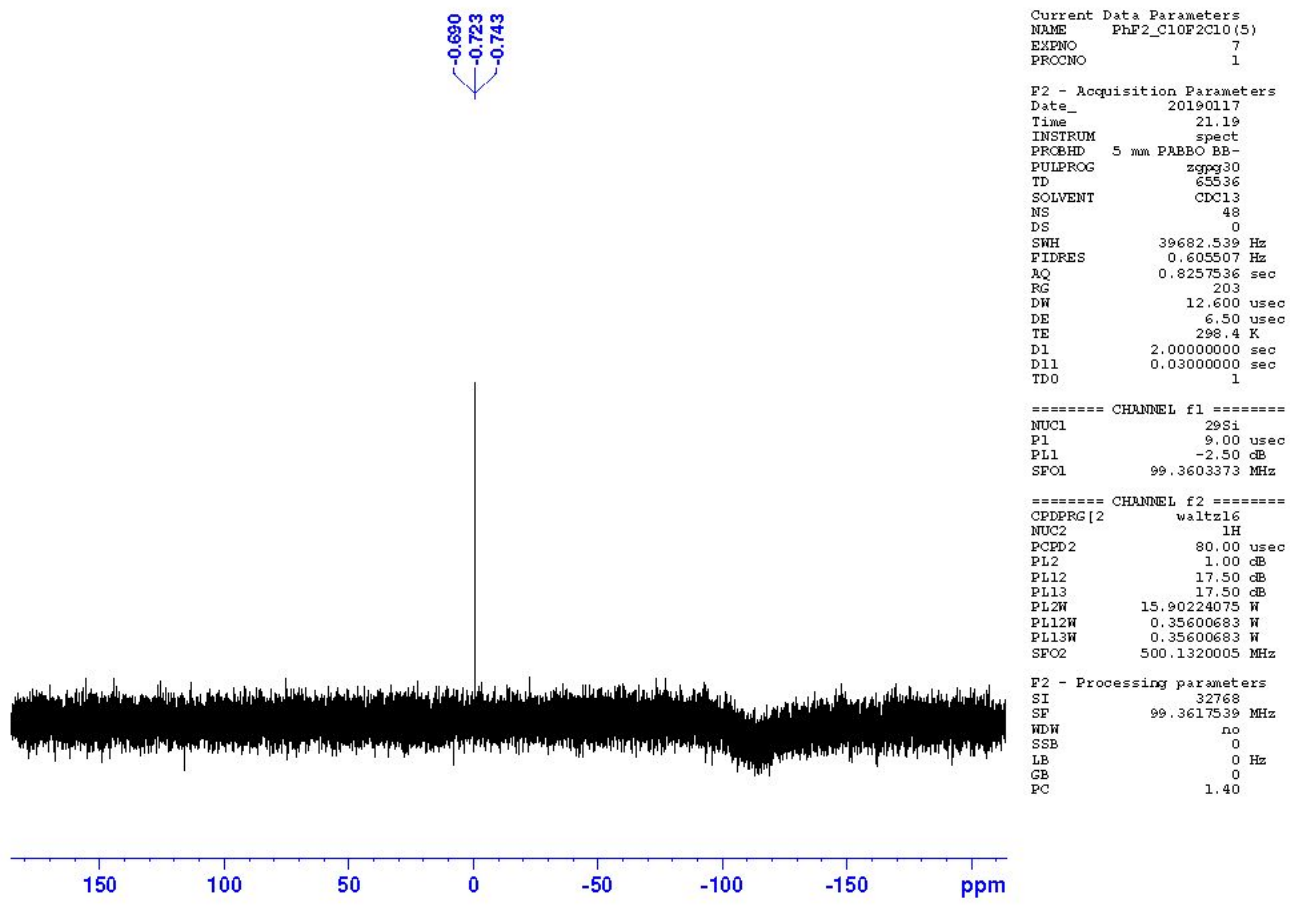

Figure S8. ${ }^{29}$ Si NMR (99 MHz) spectrum of 1,4-bis(tris-9-decenylsilyl)-2,3-difluorobenzene (2) in $\mathrm{CDCl}_{3}$. 


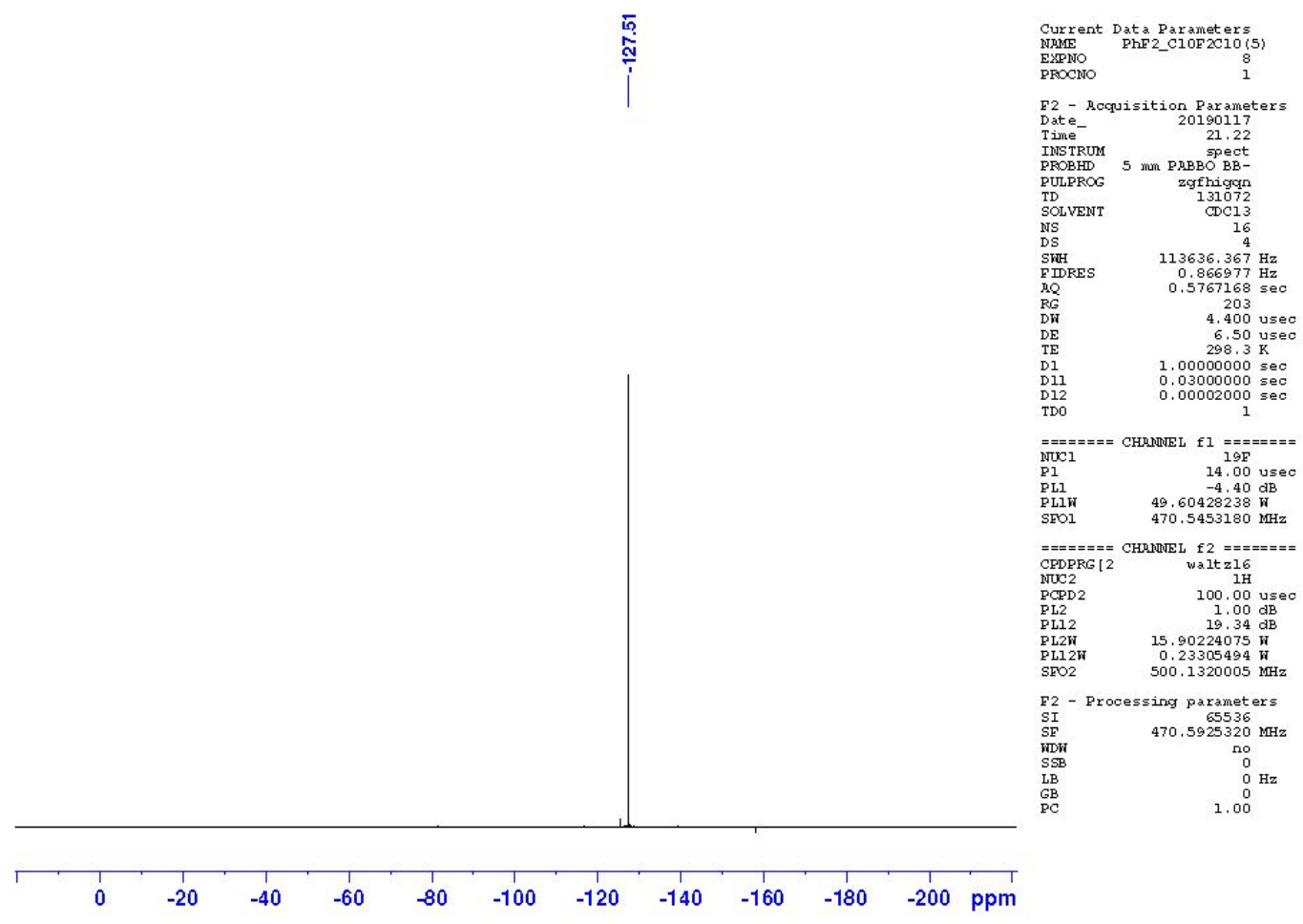

Figure S9. ${ }^{19} \mathrm{~F}$ NMR (471 MHz) spectrum of 1,4-bis(tris-9-decenylsilyl)-2,3-difluorobenzene (2) in $\mathrm{CDCl}_{3}$.

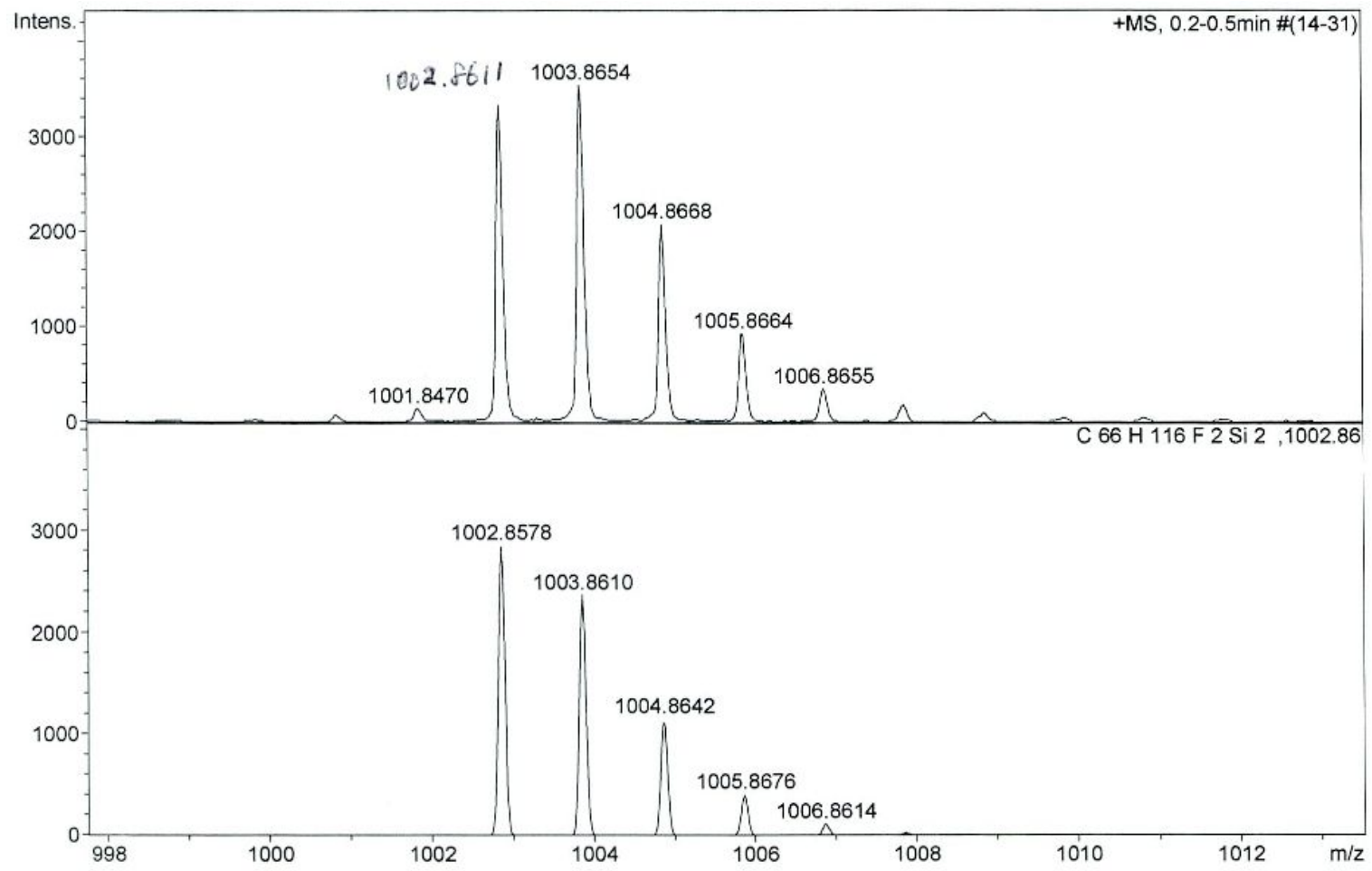

Figure S10. HRMS spectrum of 1,4-bis(tris-9-decenylsilyl)-2,3-difluorobenzene (2) (ESI, positive). Top: obsd. Bottom: sim. 

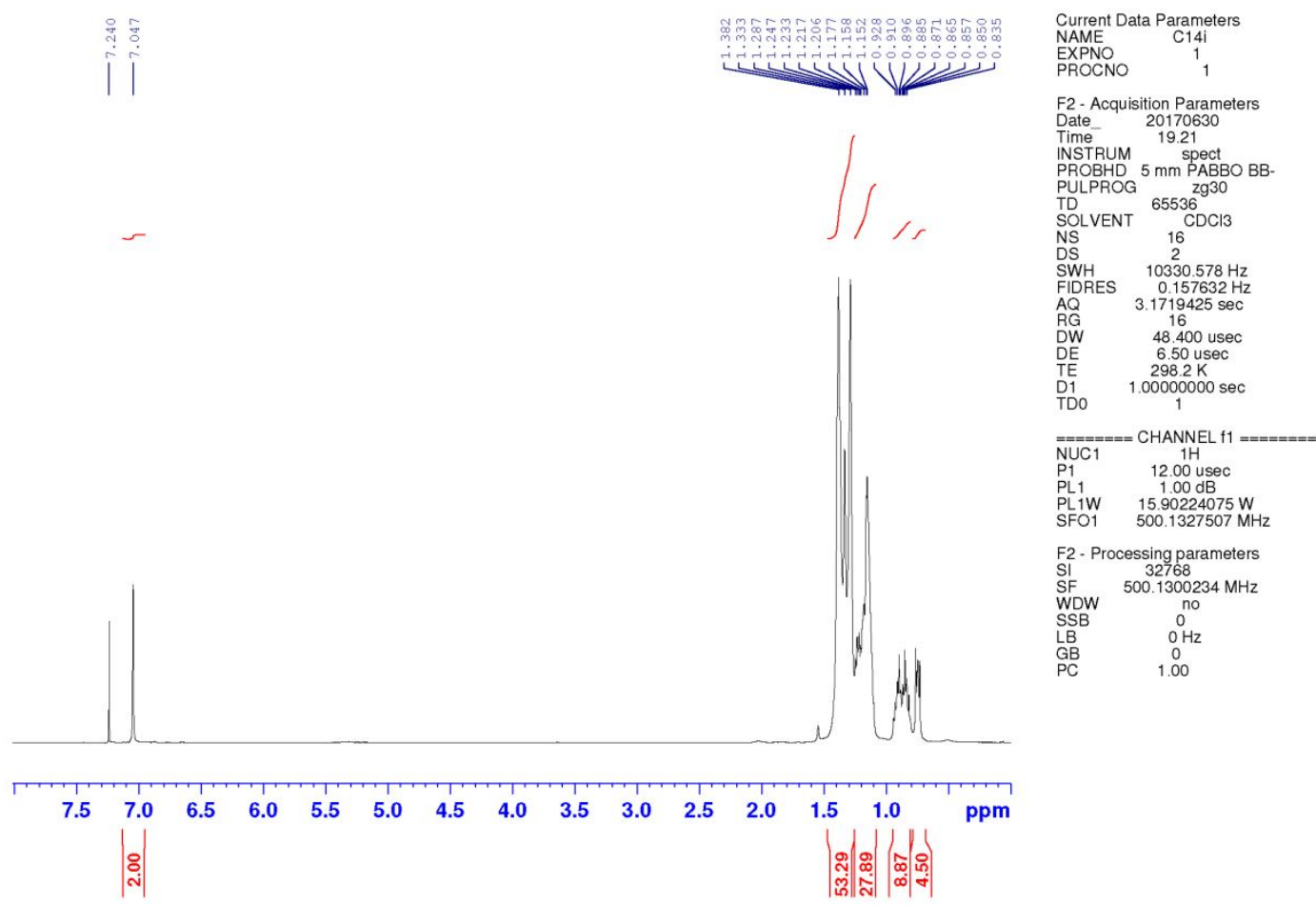

Figure S11. ${ }^{1} \mathrm{H}$ NMR (500 MHz) spectrum of molecular gyrotop C14PhF2 in $\mathrm{CDCl}_{3}$.
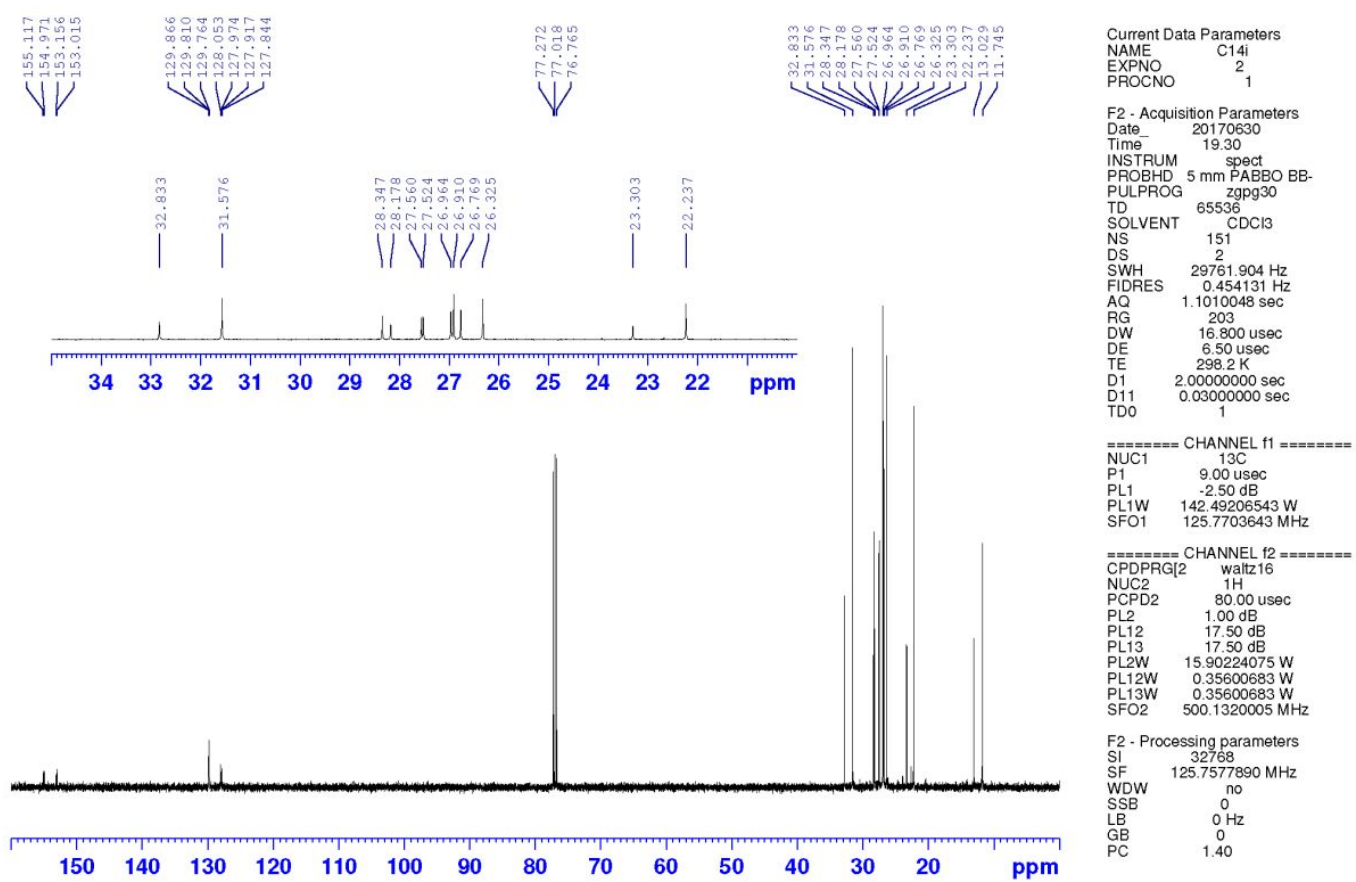

Figure S12. ${ }^{13} \mathrm{C}$ NMR (126 MHz) spectrum of molecular gyrotop C14PhF2 in $\mathrm{CDCl}_{3}$. 


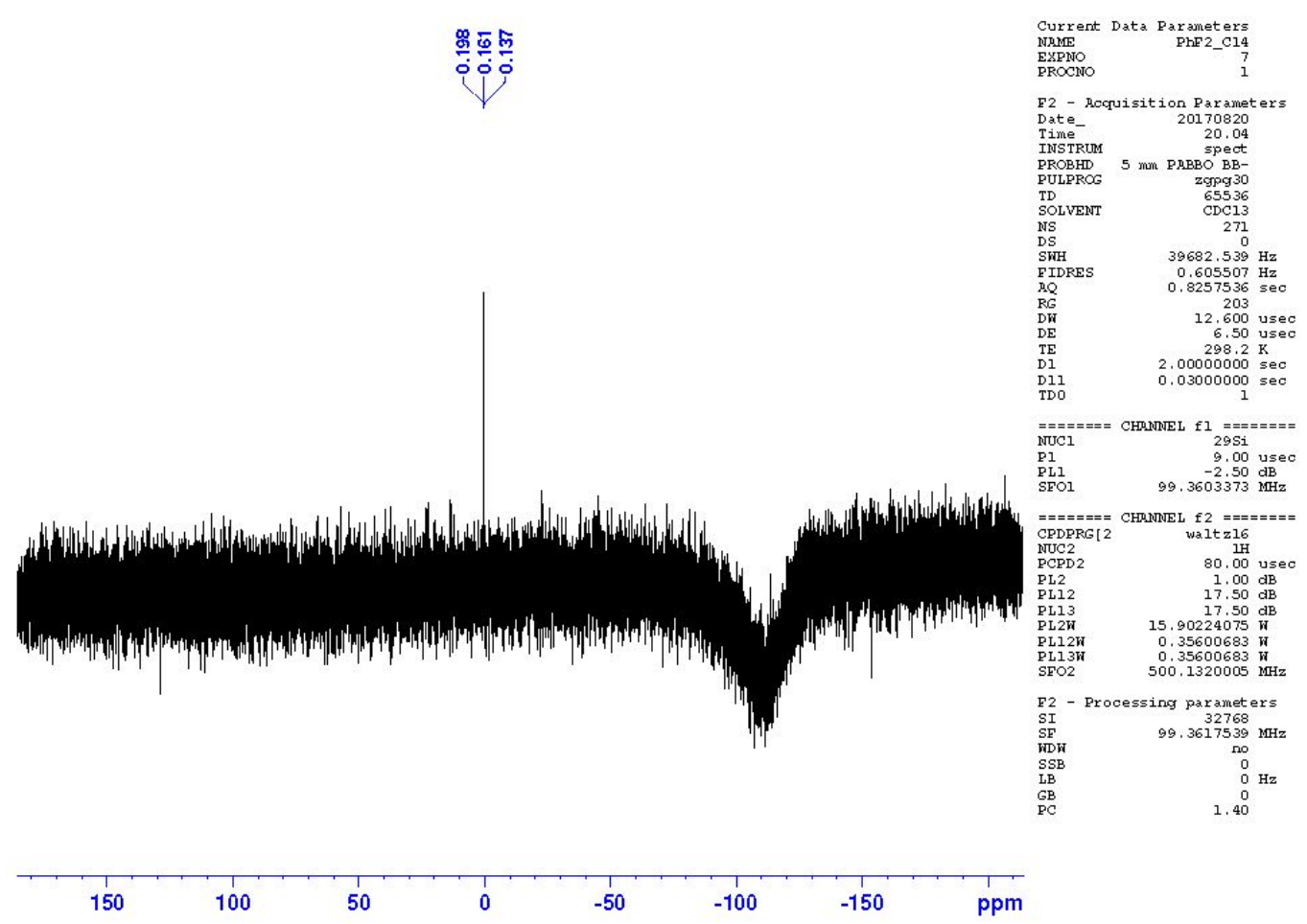

Figure S13. ${ }^{29} \mathrm{Si}$ NMR (99 MHz) spectrum of molecular gyrotop C14PhF2 in $\mathrm{CDCl}_{3}$.

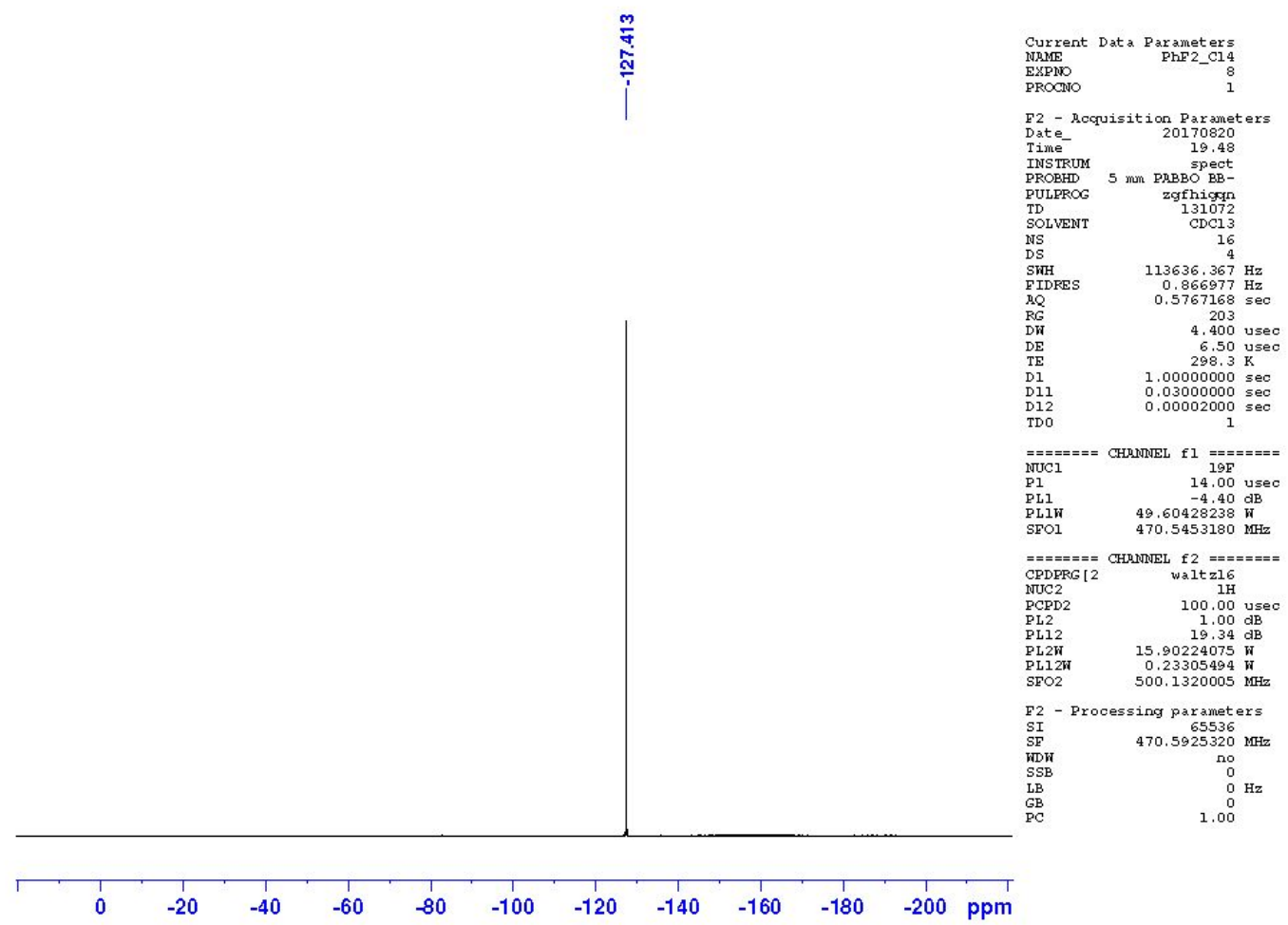

Figure S14. ${ }^{19} \mathrm{~F}$ NMR $(471 \mathrm{MHz})$ spectrum of molecular gyrotop C14PhF2 in $\mathrm{CDCl}_{3}$. 


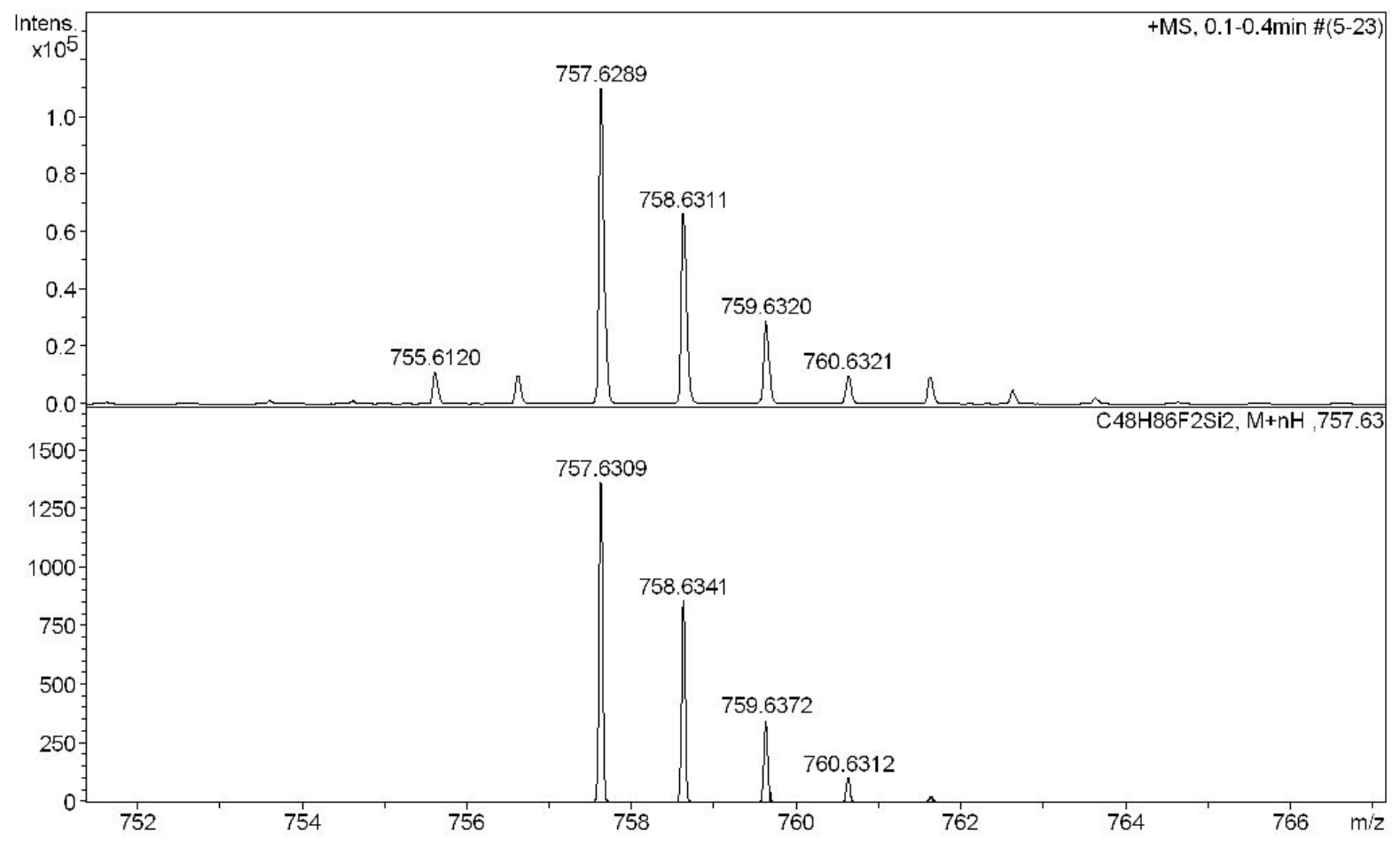

Figure S15. HRMS spectrum of molecular gyrotop C14PhF2 (ESI, positive). Top: obsd. Bottom: sim.

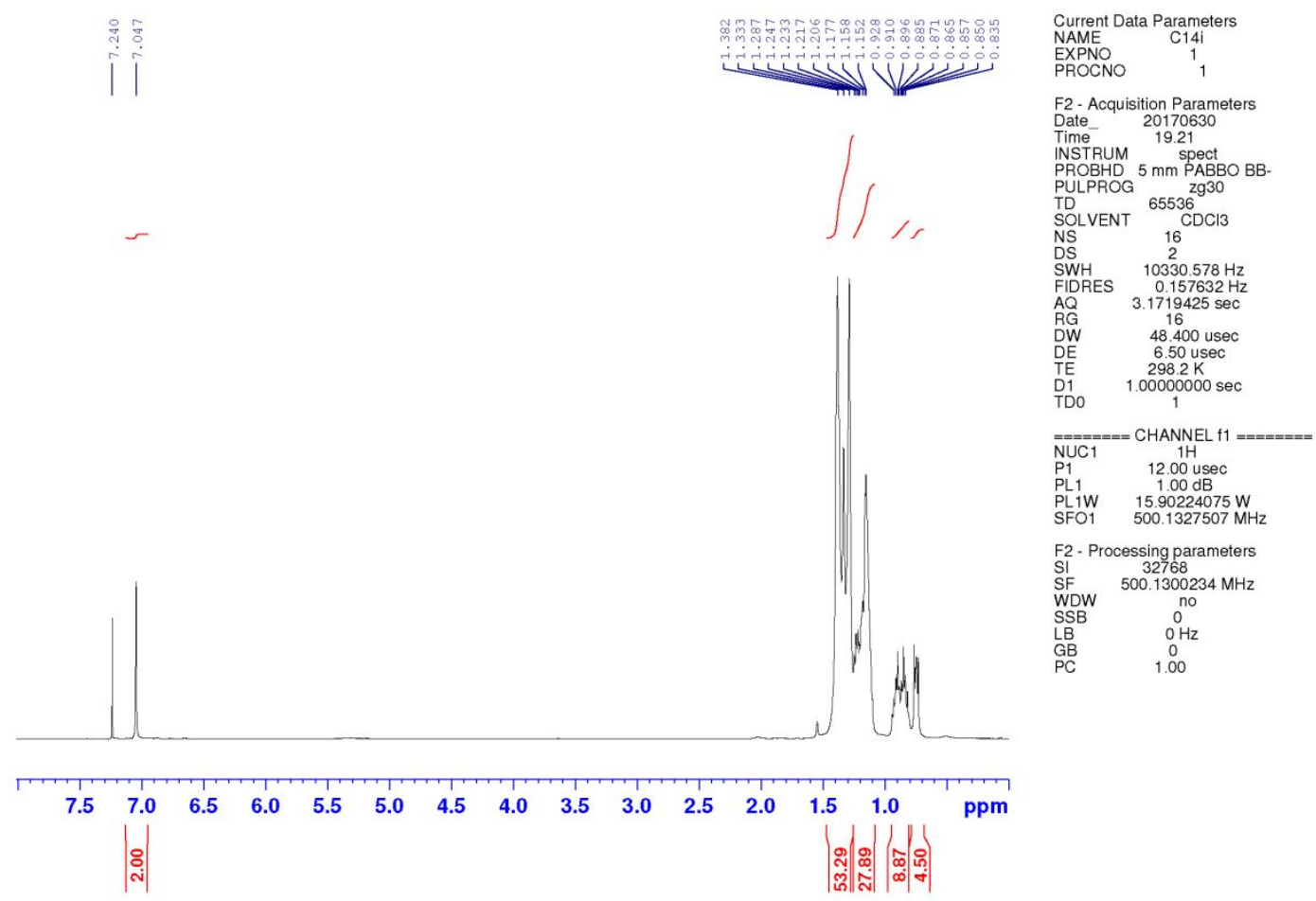

Figure S16. ${ }^{1} \mathrm{H}$ NMR (500 MHz) spectrum of molecular gyrotop isomer C14iPhF2 in $\mathrm{CDCl}_{3}$. 


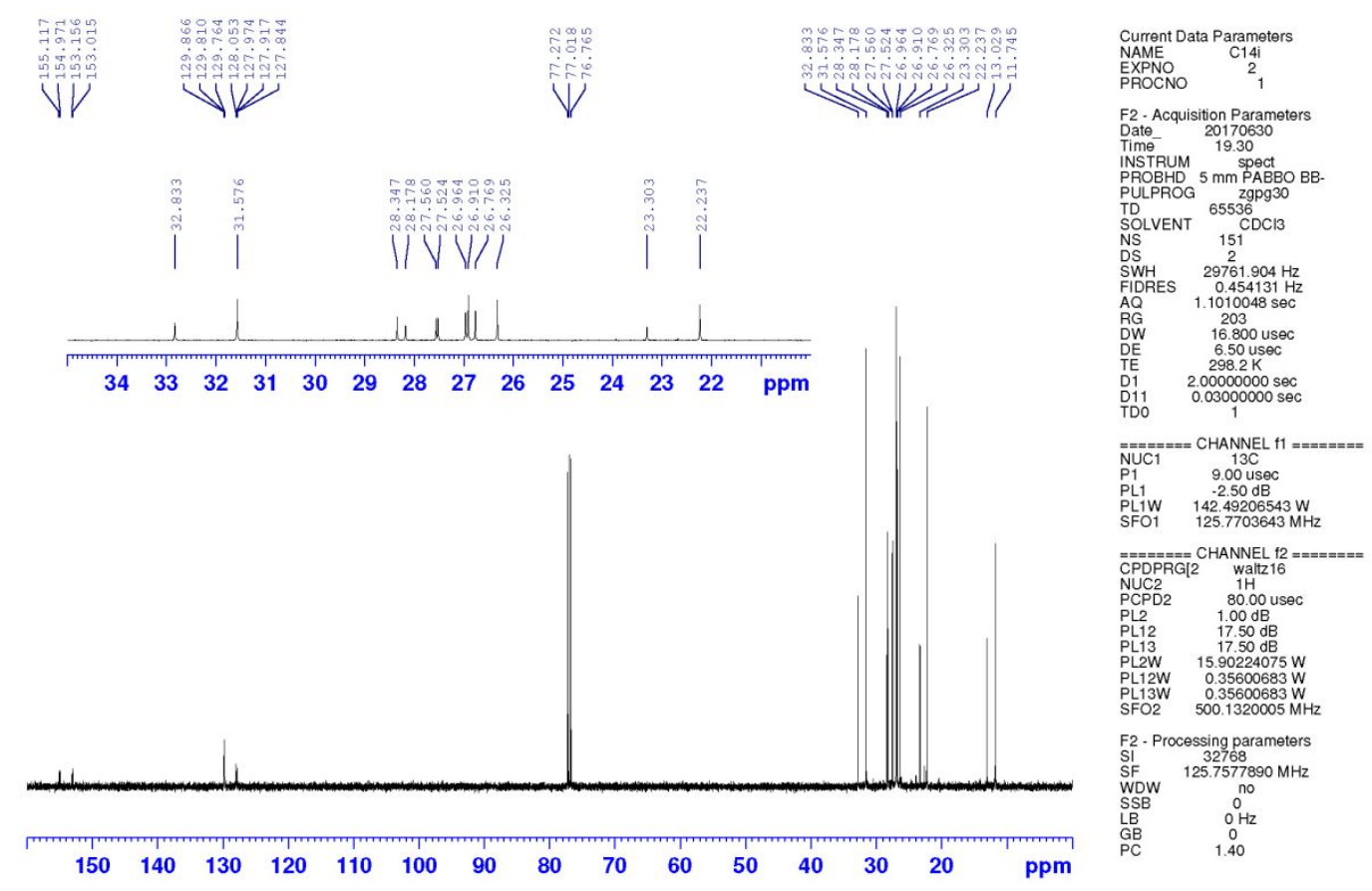

Figure S17. ${ }^{13} \mathrm{C}$ NMR (126 MHz) spectrum of molecular gyrotop isomer $\mathbf{C 1 4 i P h F 2}$ in $\mathrm{CDCl}_{3}$.

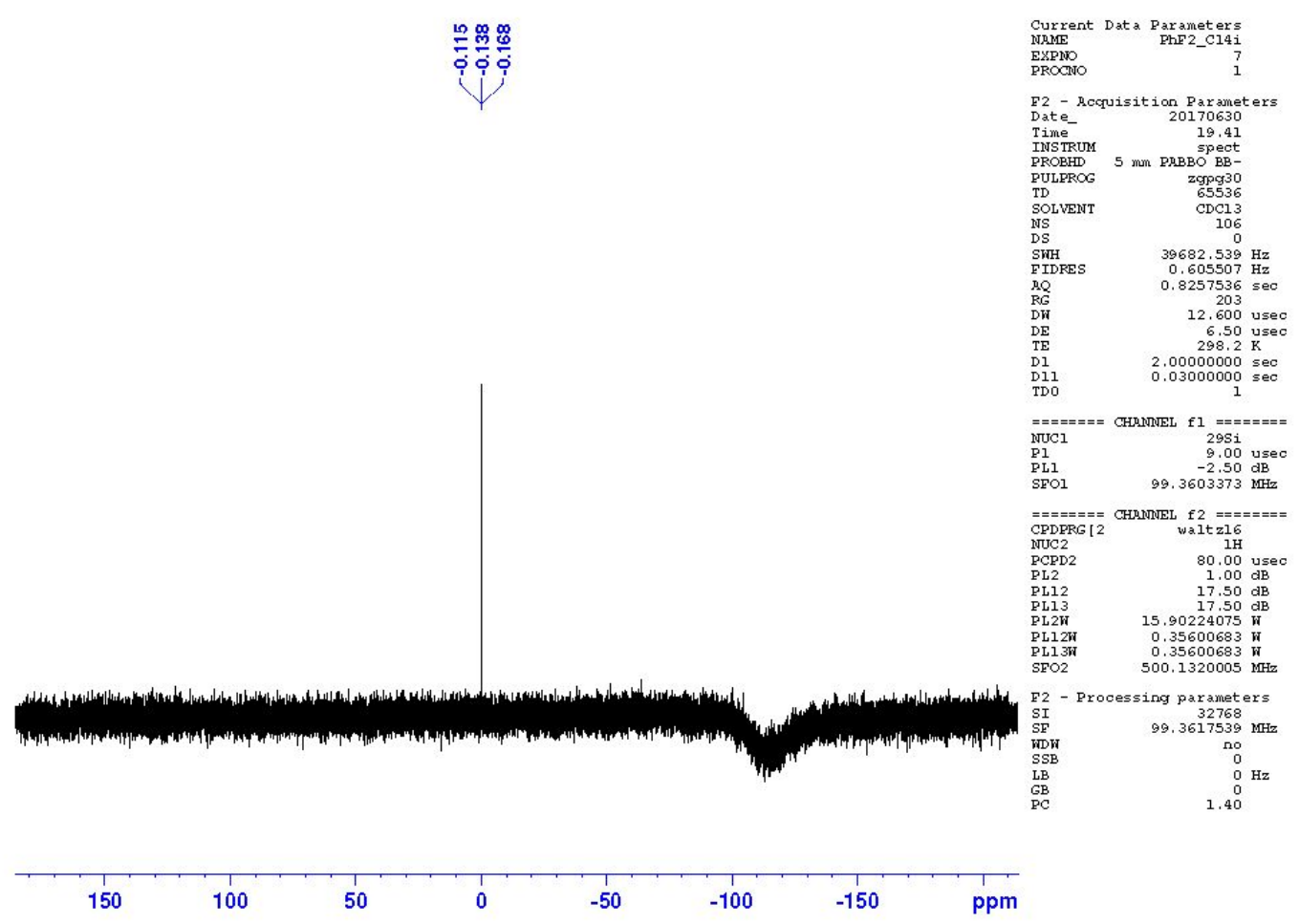

Figure S18. ${ }^{29} \mathrm{Si}$ NMR (99 MHz) spectrum of molecular gyrotop isomer C14iPhF2 in $\mathrm{CDCl}_{3}$. 


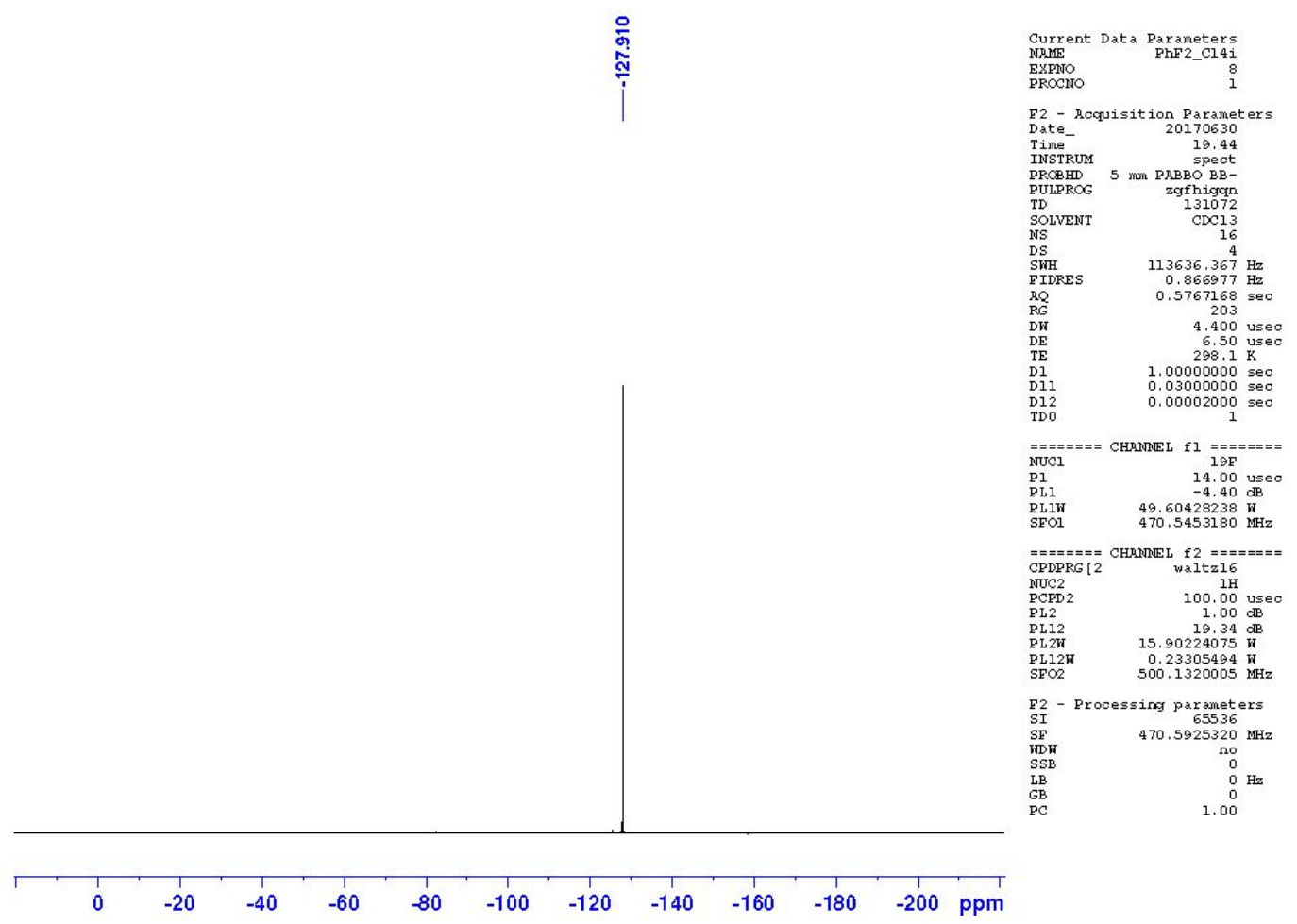

Figure S19. ${ }^{19} \mathrm{~F}$ NMR (471 MHz) spectrum of molecular gyrotop isomer C14iPhF2 in $\mathrm{CDCl}_{3}$.

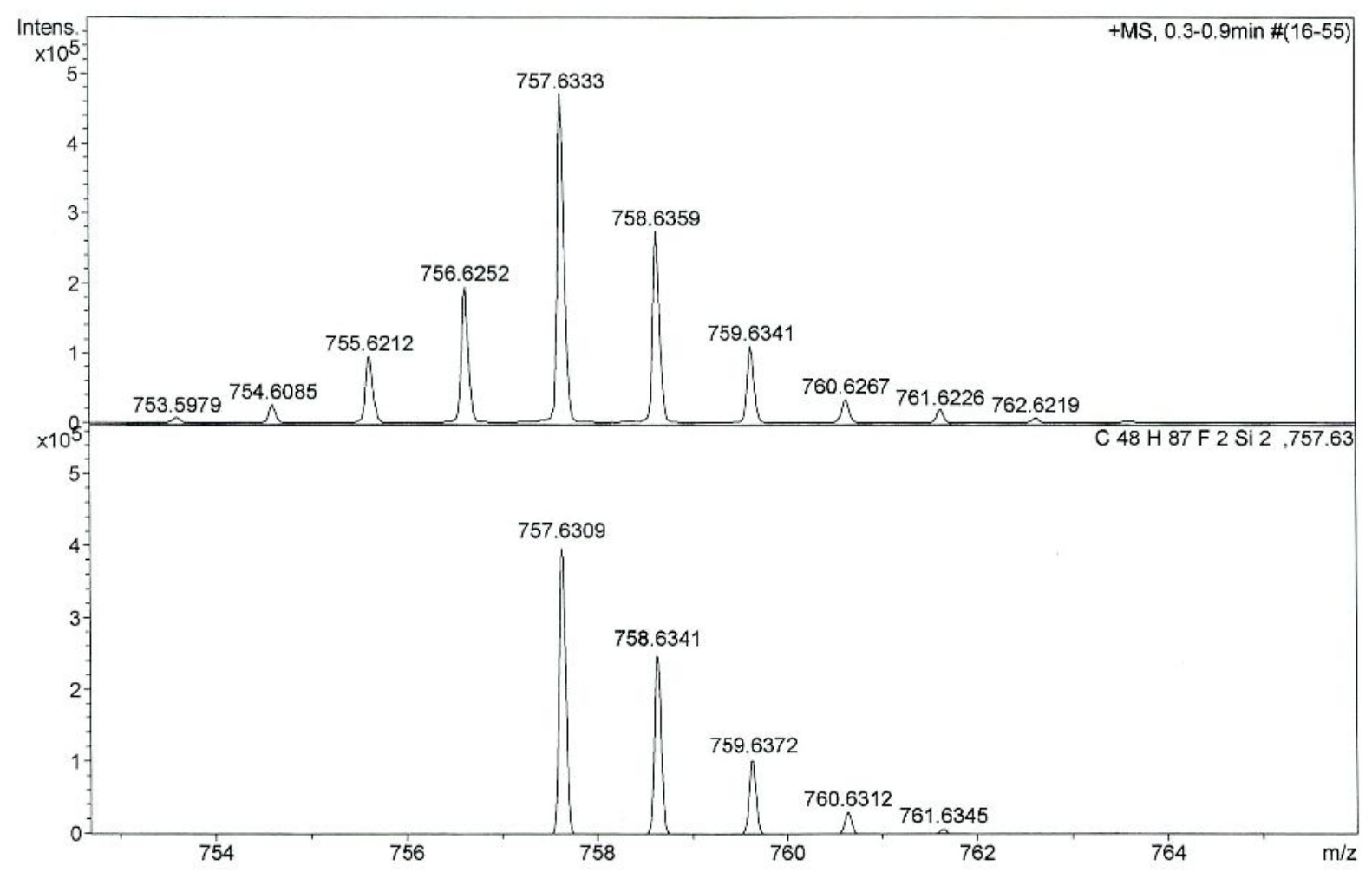

Figure S20. HRMS spectrum of molecular gyrotop isomer C14iPhF2 (ESI, positive). Top: obsd. Bottom: sim. 


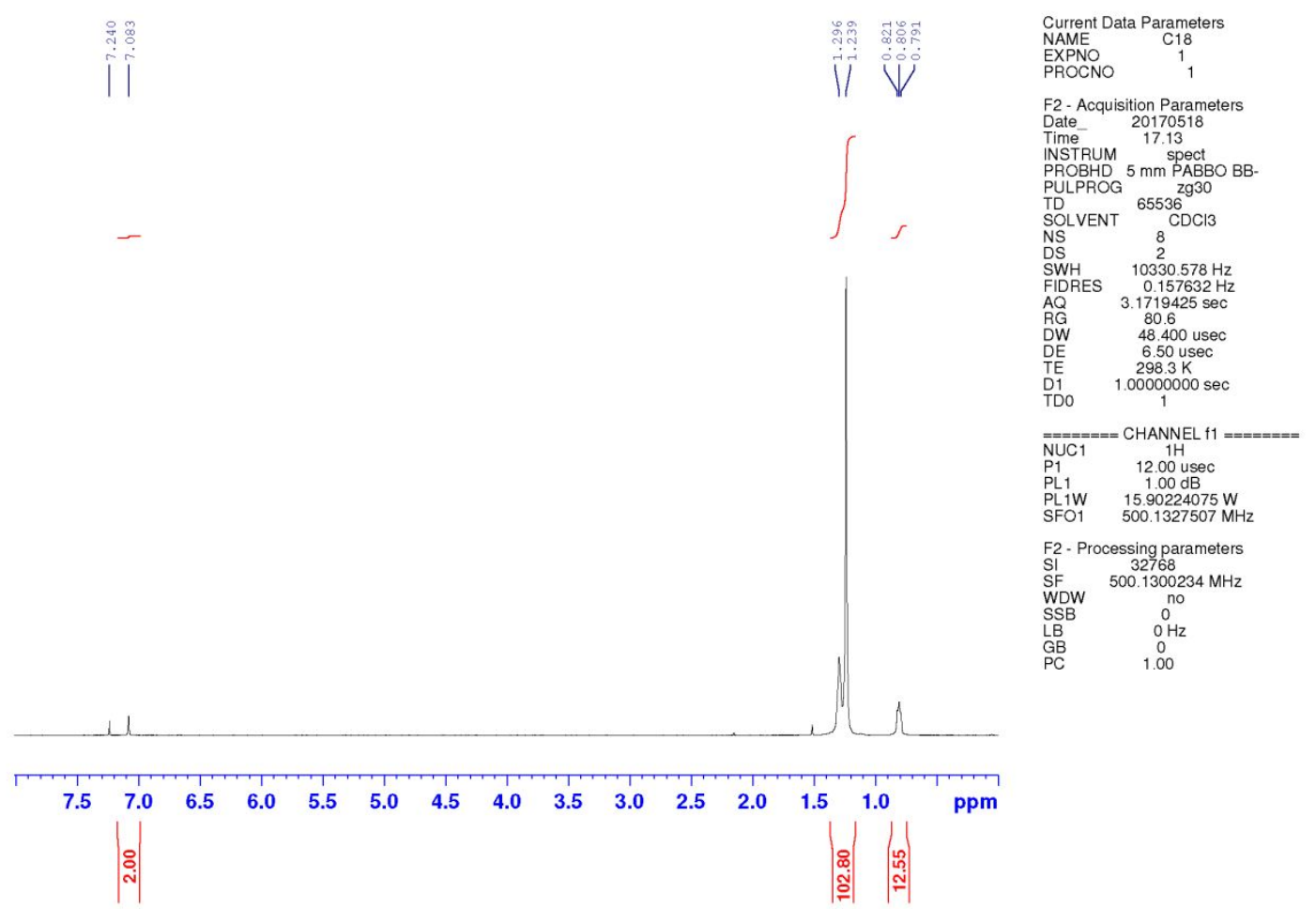

Figure S21. ${ }^{1} \mathrm{H}$ NMR (500 MHz) spectrum of molecular gyrotop C18PhF2 in $\mathrm{CDCl}_{3}$.
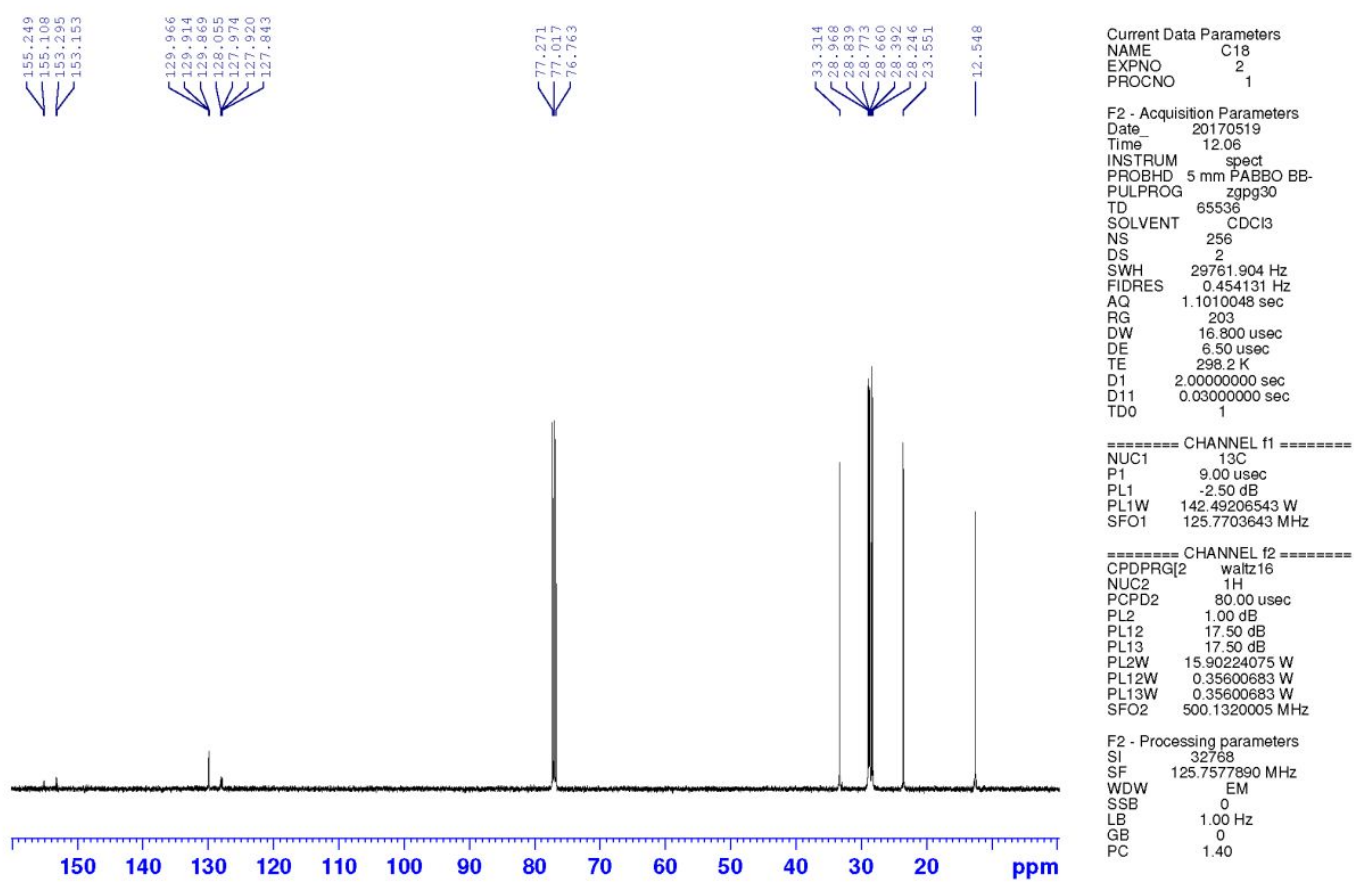

Figure S22. ${ }^{13} \mathrm{C}$ NMR (126 MHz) spectrum of molecular gyrotop C18PhF2 in $\mathrm{CDCl}_{3}$. 


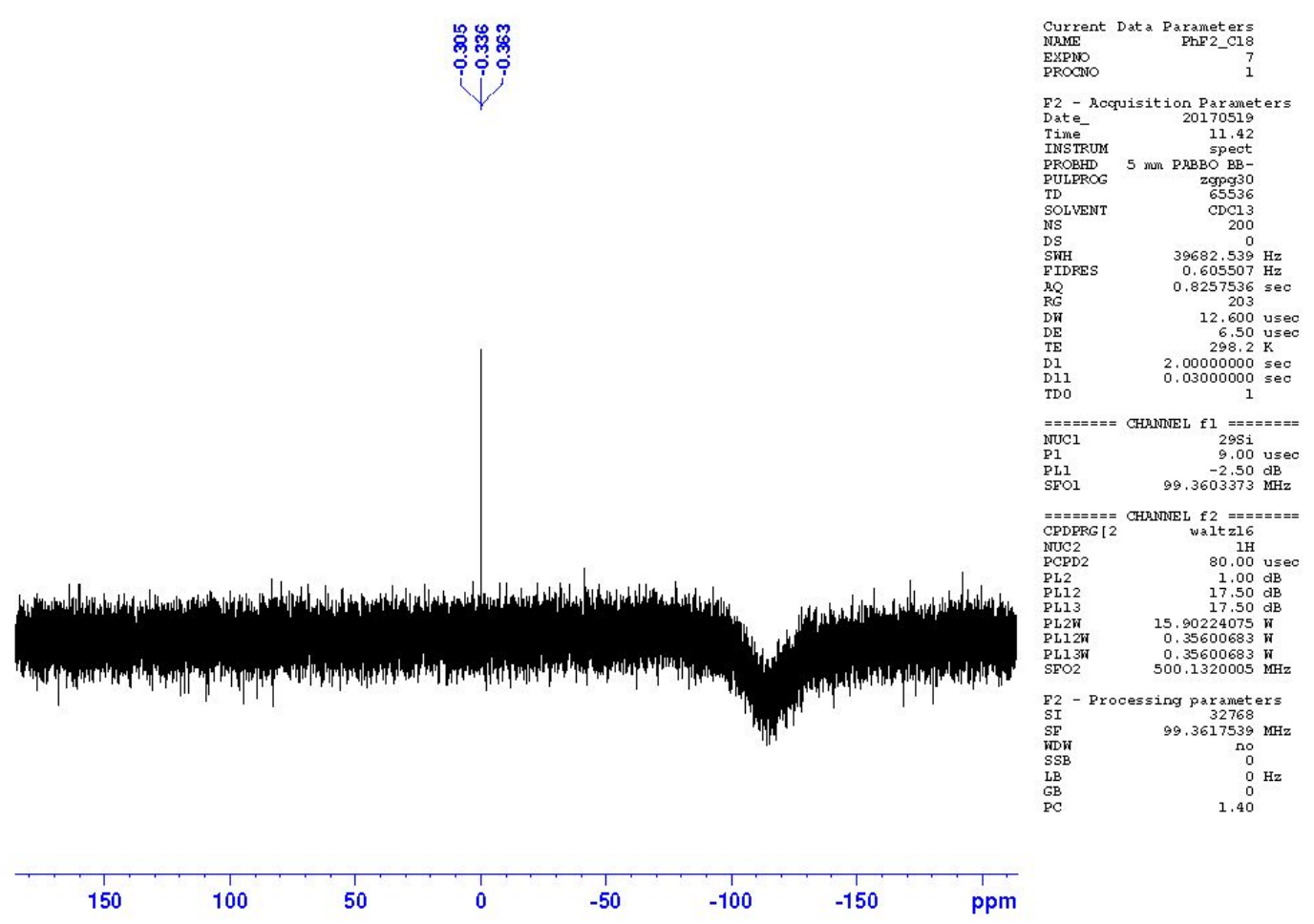

Figure S23. ${ }^{29} \mathrm{Si}$ NMR (99 MHz) spectrum of molecular gyrotop C18PhF2 in $\mathrm{CDCl}_{3}$.

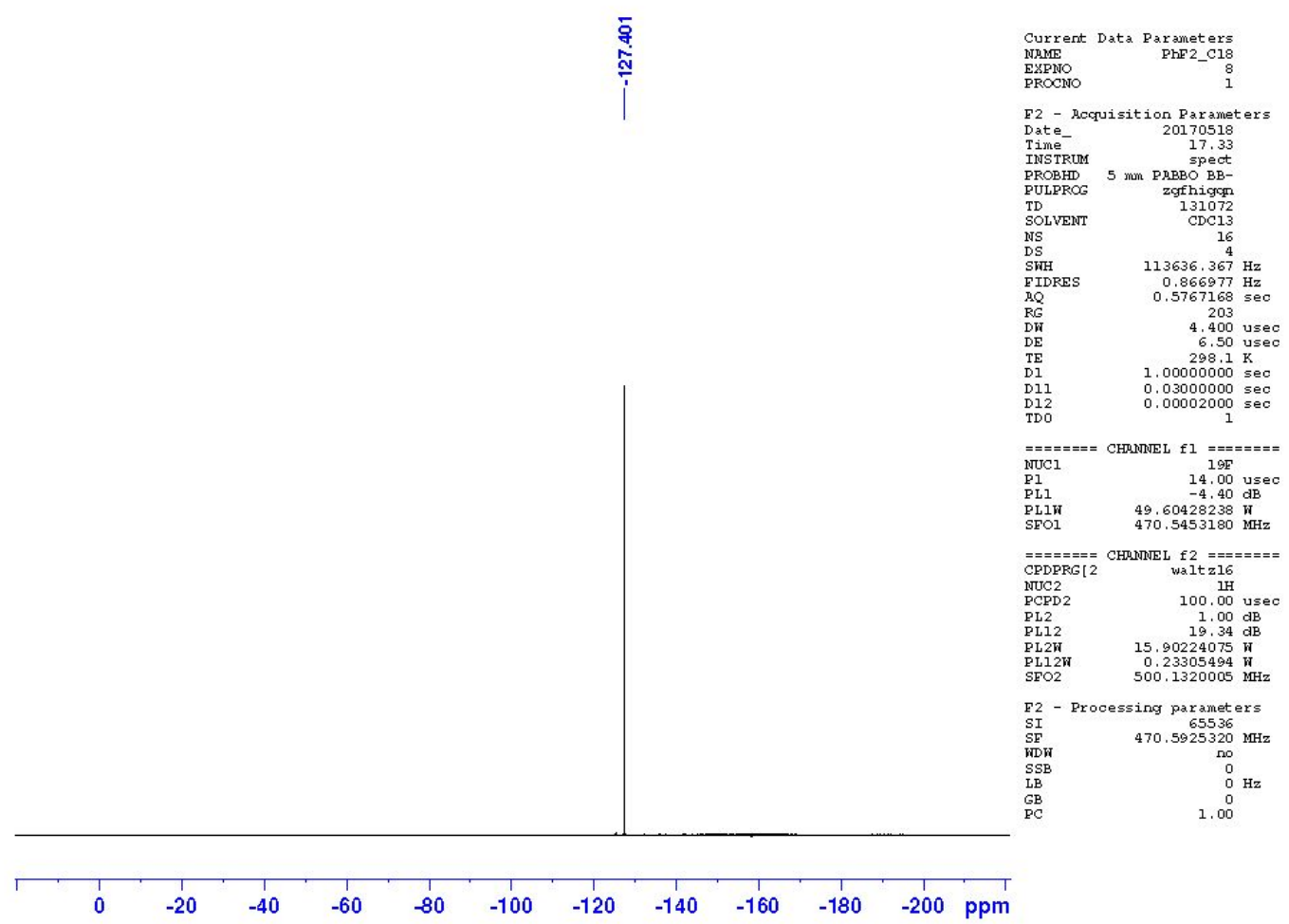

Figure S24. ${ }^{19} \mathrm{~F}$ NMR $(471 \mathrm{MHz})$ spectrum of molecular gyrotop C18PhF2 in $\mathrm{CDCl}_{3}$. 


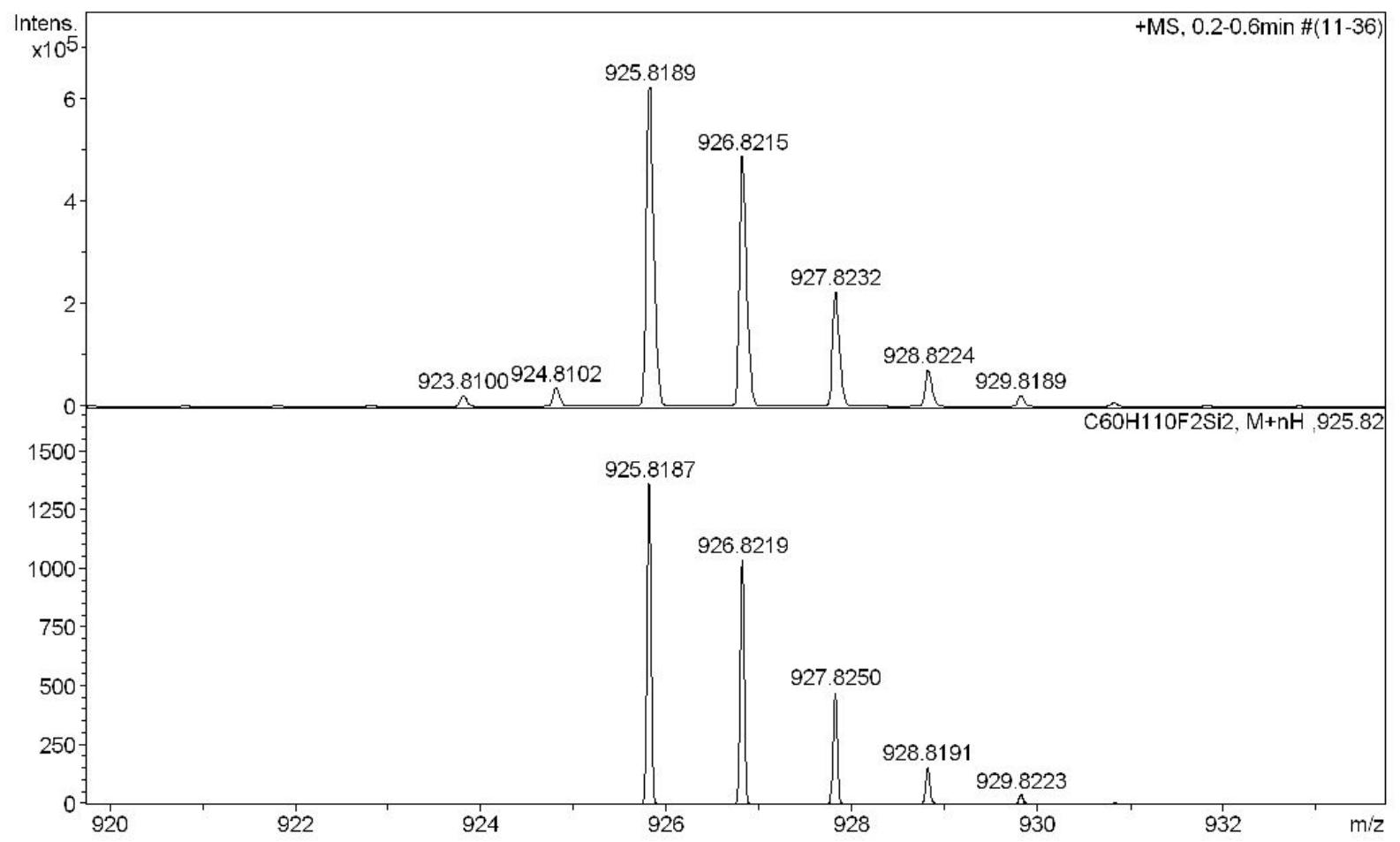

Figure S25. HRMS spectrum of molecular gyrotop C18PhF2 (APCI, positive). Top: obsd. Bottom: sim.

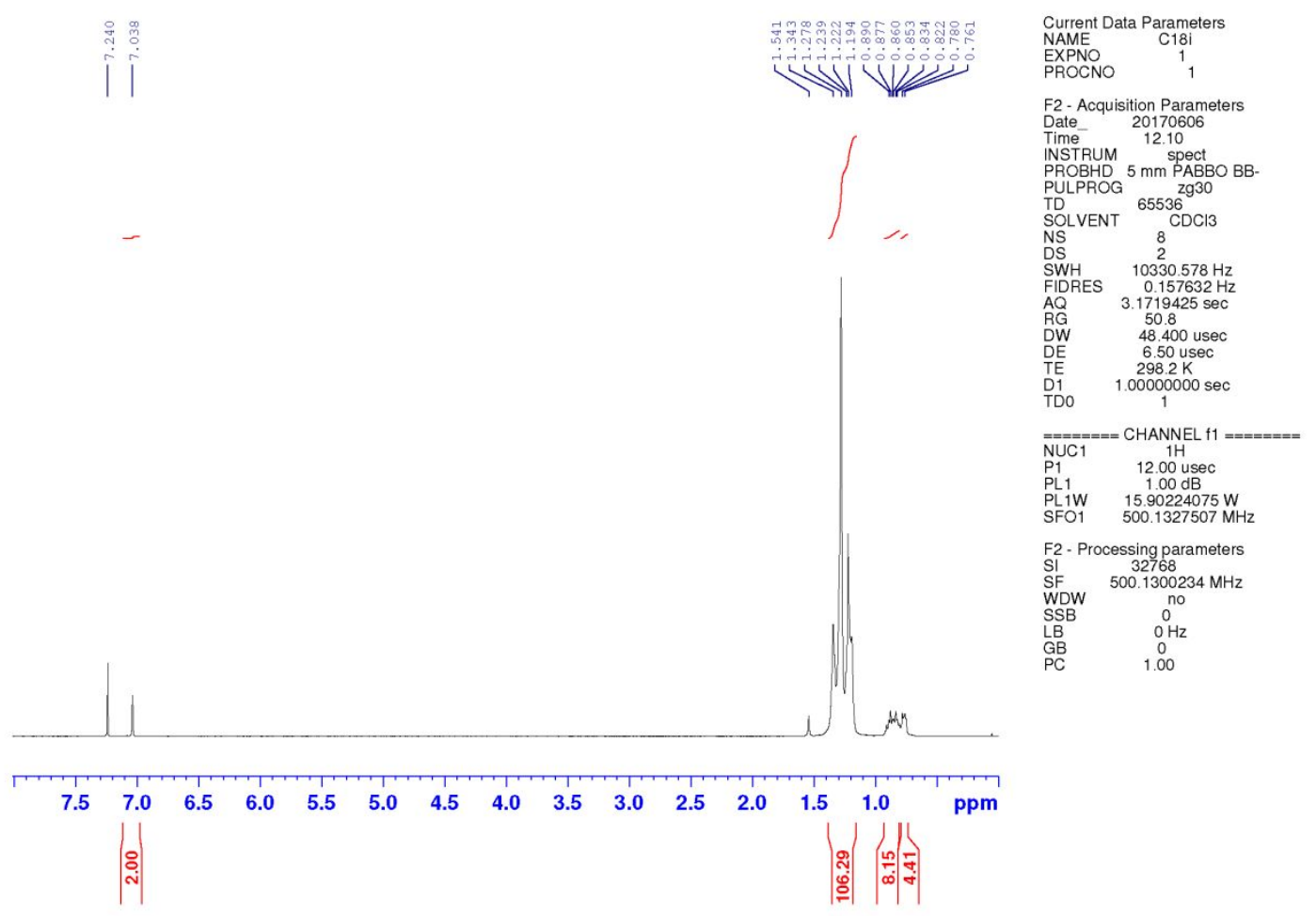

Figure S26. ${ }^{1} \mathrm{H}$ NMR ( $\left.500 \mathrm{MHz}\right)$ spectrum of molecular gyrotop isomer $\mathbf{C 1 8 i P h F 2}$ in $\mathrm{CDCl}_{3}$. 


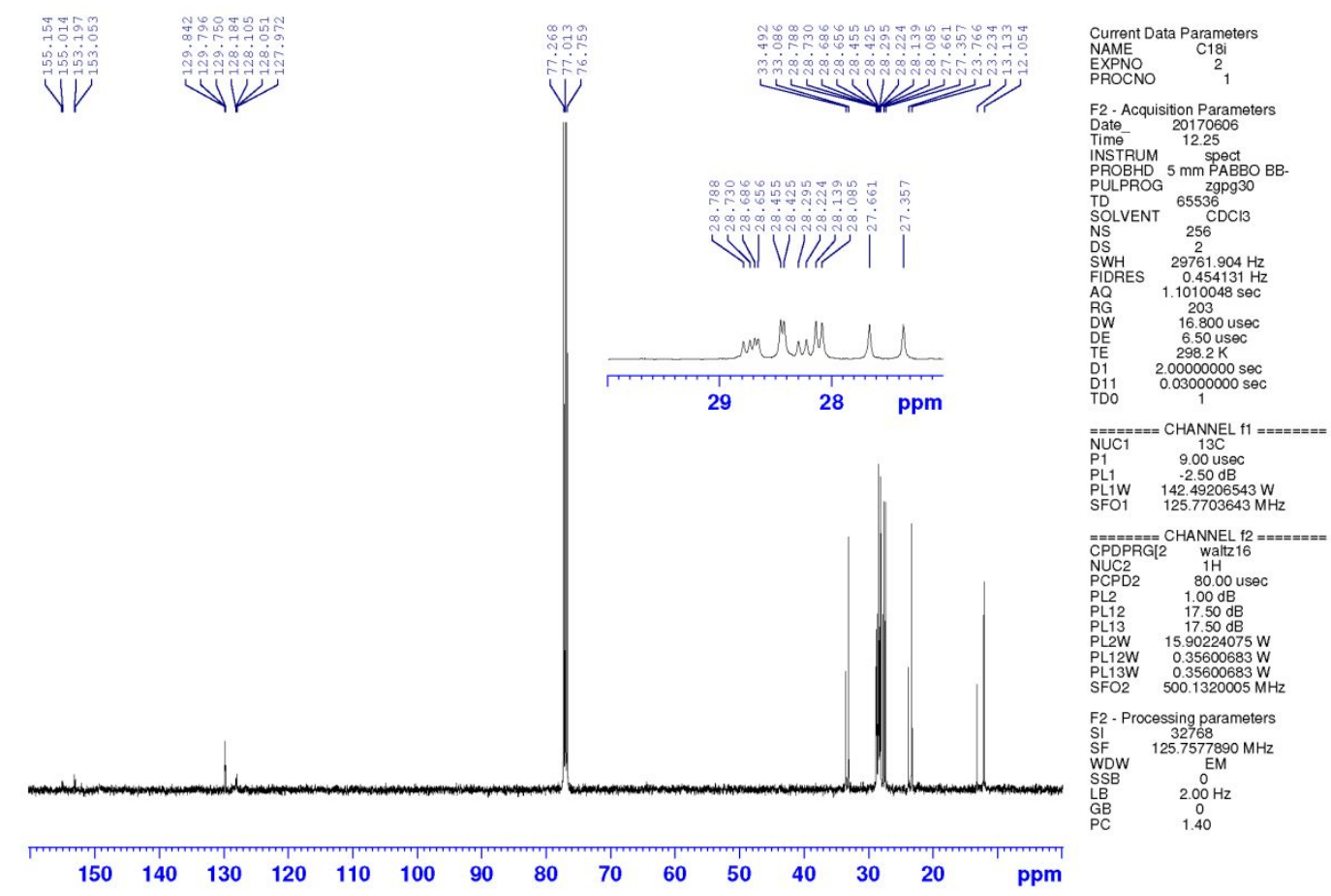

Figure S27. ${ }^{13} \mathrm{C}$ NMR (126 MHz) spectrum of molecular gyrotop isomer $\mathbf{C 1 8 i P h F 2}$ in $\mathrm{CDCl}_{3}$.

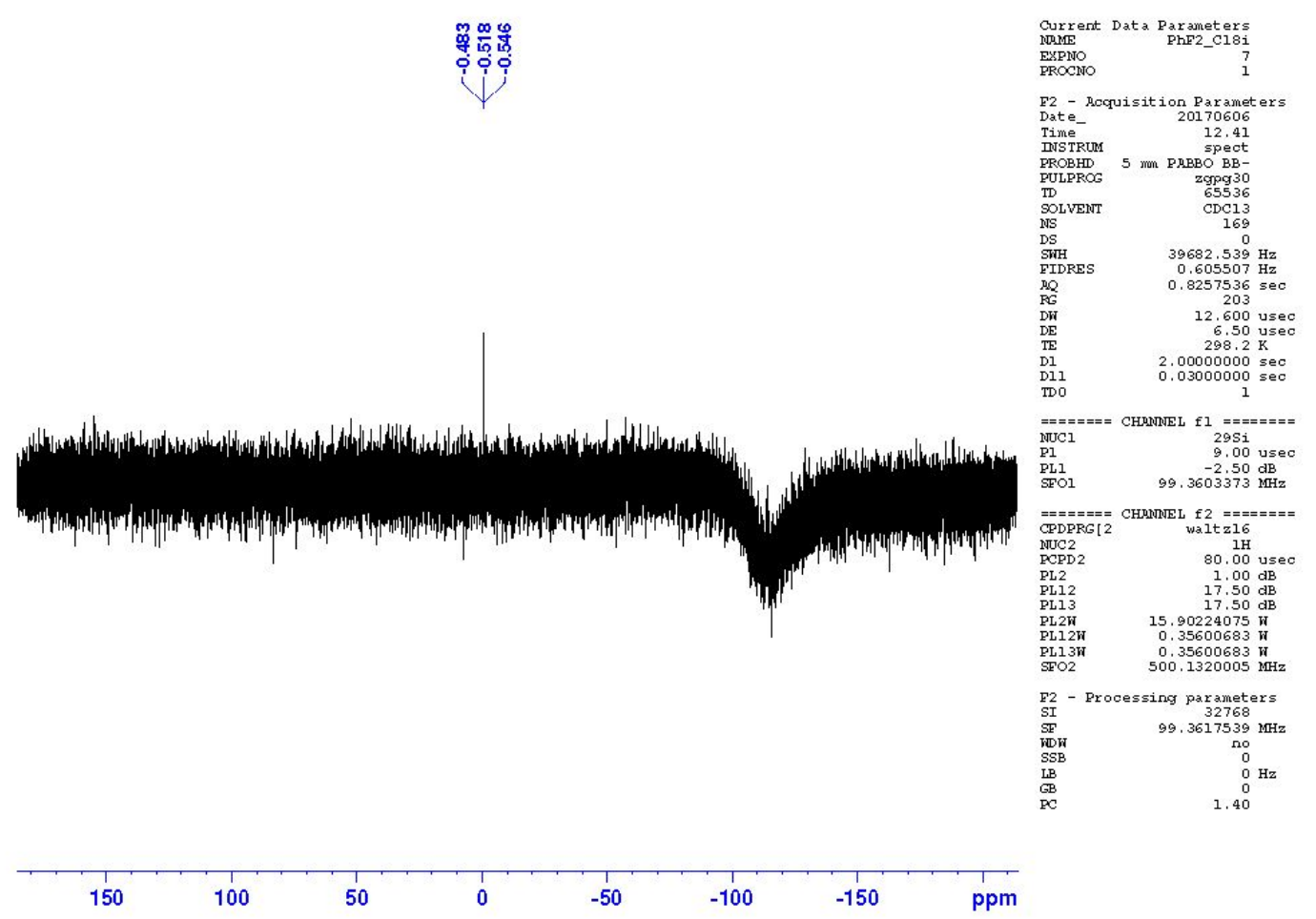

Figure S28. ${ }^{29} \mathrm{Si}$ NMR (99 MHz) spectrum of molecular gyrotop isomer $\mathbf{C 1 8 i P h F 2}$ in $\mathrm{CDCl}_{3}$. 


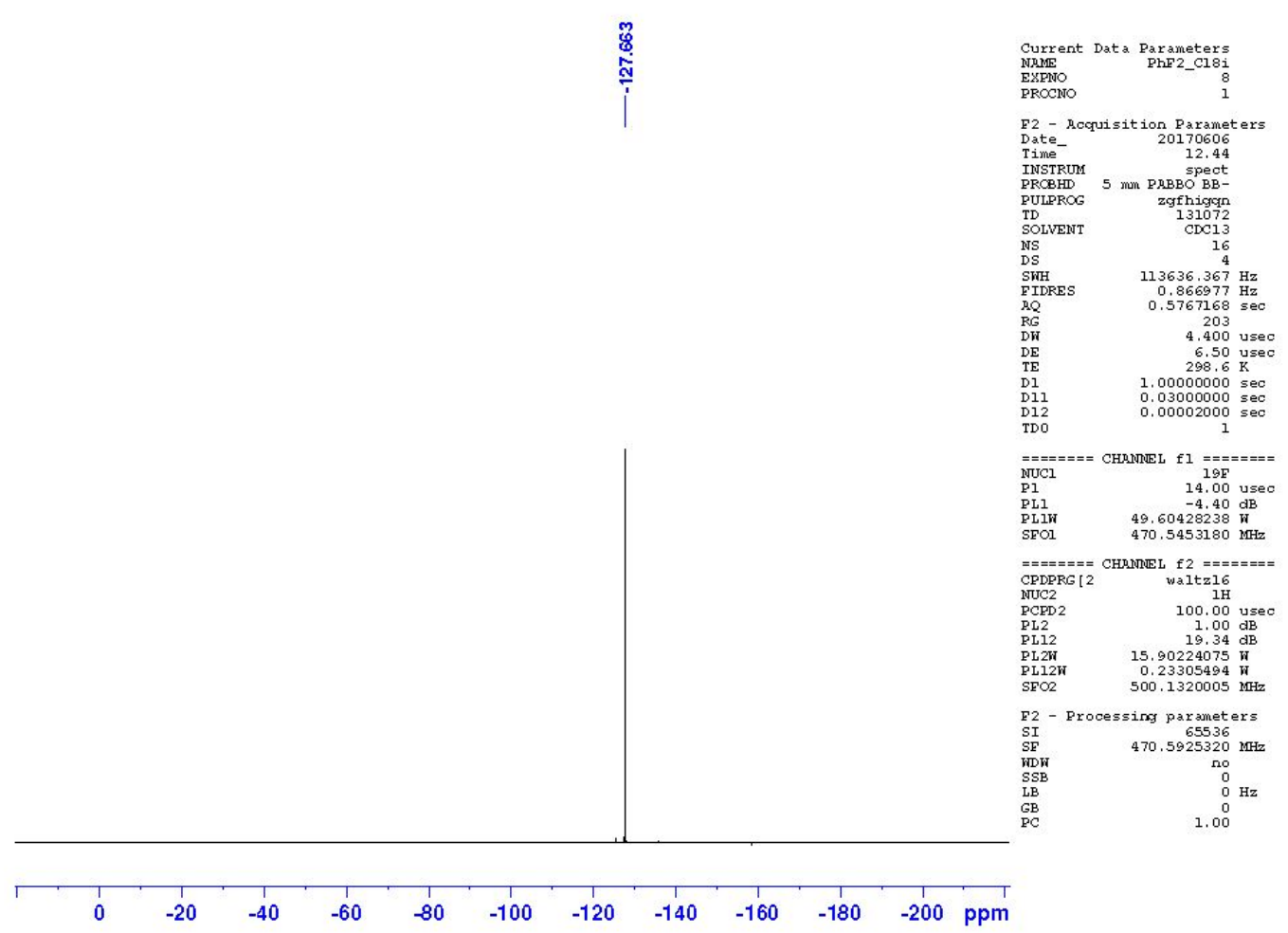

Figure S29. ${ }^{19} \mathrm{~F}$ NMR (471 MHz) spectrum of molecular gyrotop isomer $\mathbf{C 1 8 i P h F 2}$ in $\mathrm{CDCl}_{3}$.

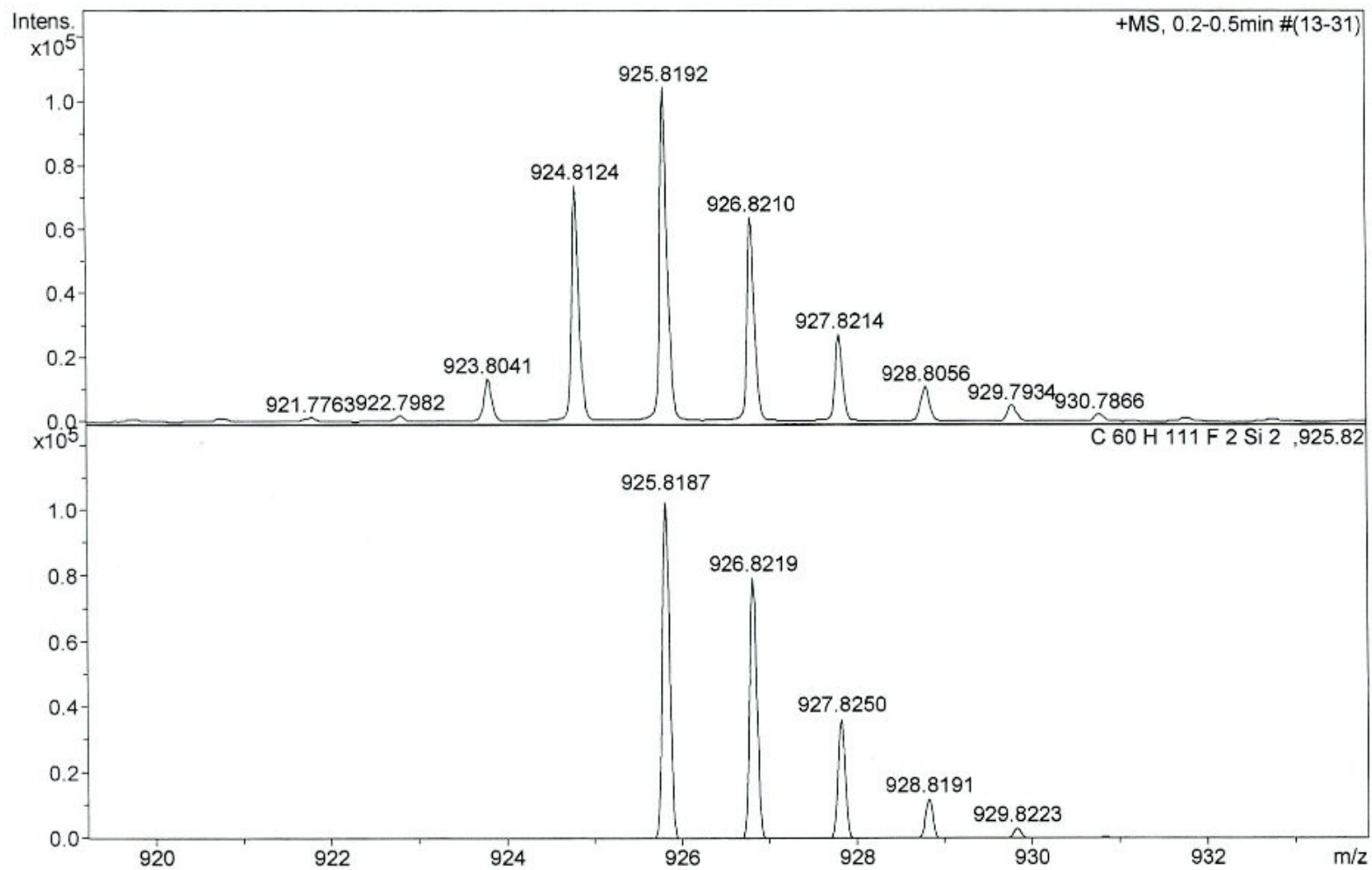

Figure S30. HRMS spectrum of molecular gyrotop isomer C18iPhF2 (ESI, positive). Top: obsd. Bottom: sim. 


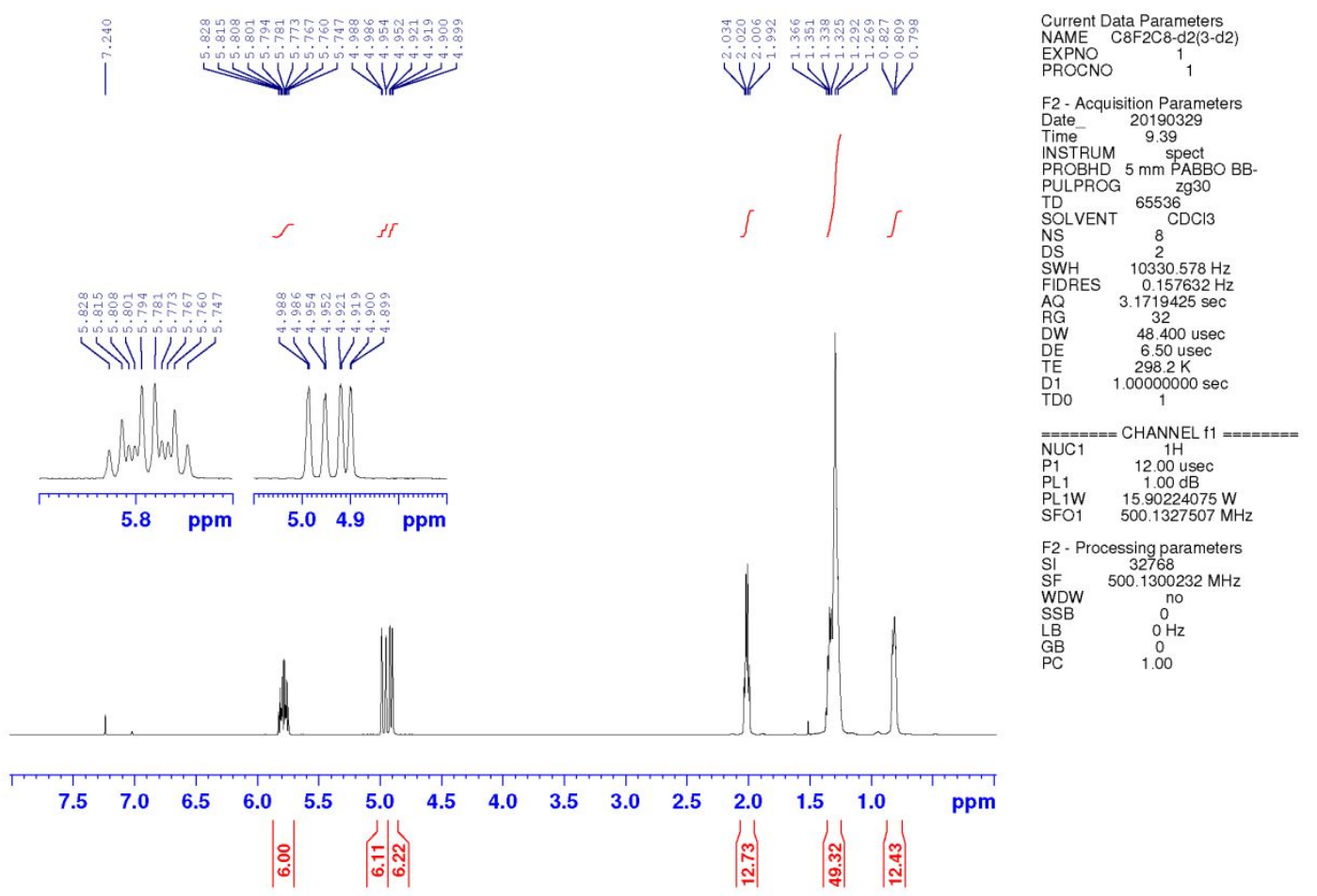

Figure S31. ${ }^{1} \mathrm{H}$ NMR (500 MHz) spectrum of 1,4-bis(tris-7-octenylsilyl)-4,5-diduterio-2,3difluorobenzene $\left(\mathbf{1}-d_{2}\right)$ in $\mathrm{CDCl}_{3}$.
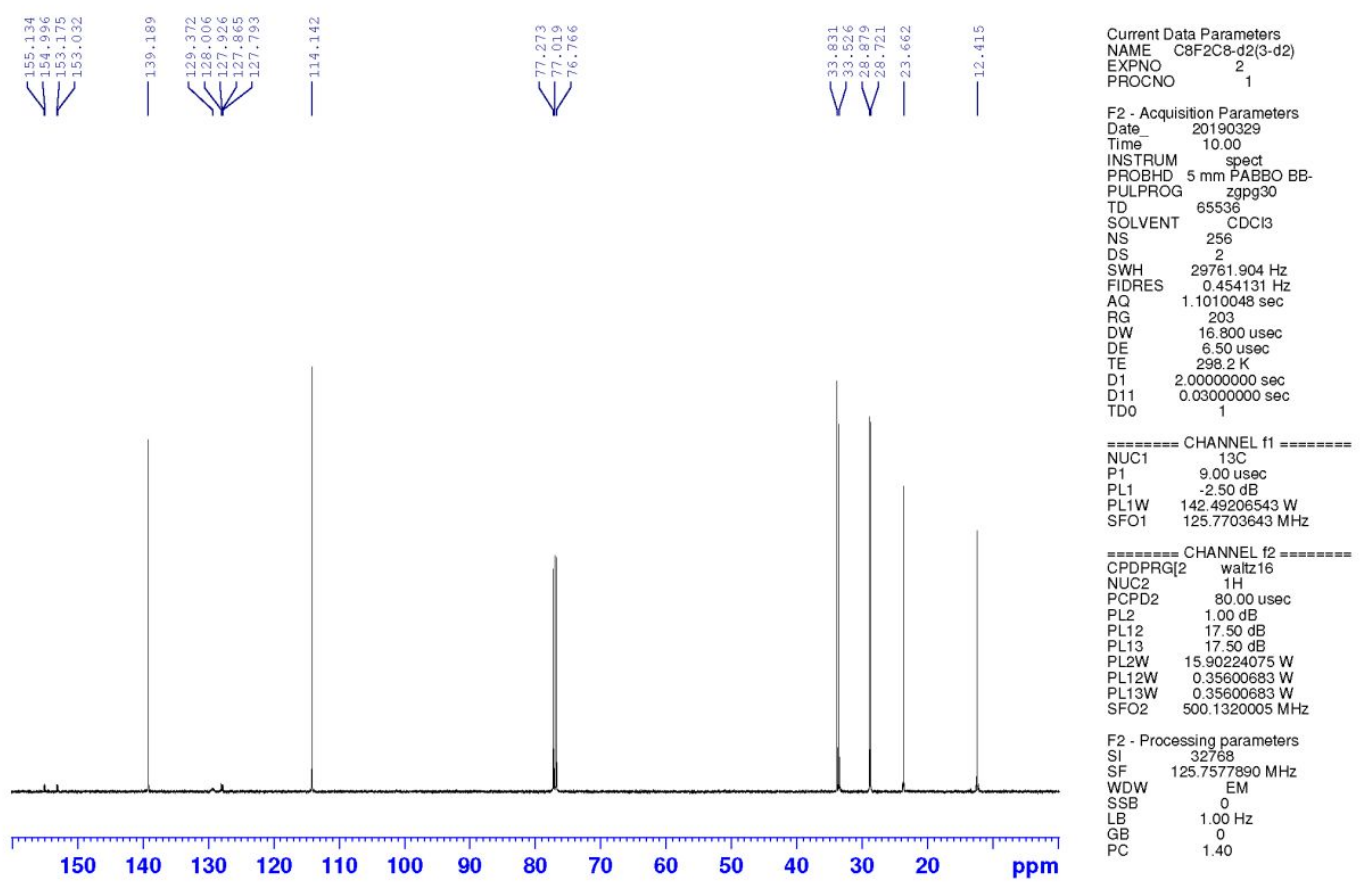

Figure S32. ${ }^{13} \mathrm{C}$ NMR (126 MHz) spectrum of 1,4-bis(tris-7-octenylsilyl)-4,5-diduterio-2,3difluorobenzene $\left(\mathbf{1}-d_{2}\right)$ in $\mathrm{CDCl}_{3}$. 


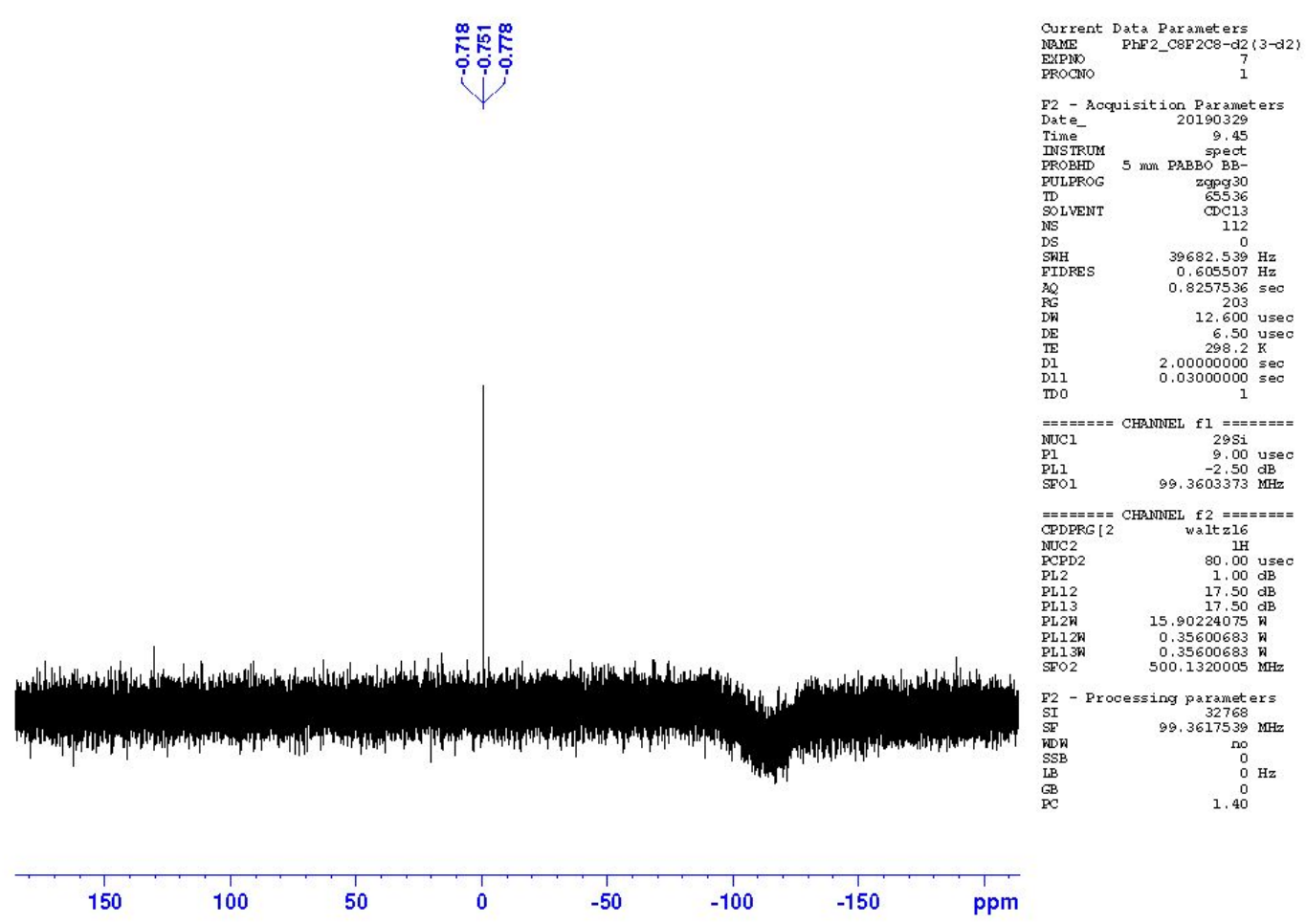

Figure S33. ${ }^{29} \mathrm{Si}$ NMR (99 MHz) spectrum of 1,4-bis(tris-7-octenylsilyl)-4,5-diduterio-2,3difluorobenzene $\left(1-d_{2}\right)$ in $\mathrm{CDCl}_{3}$.

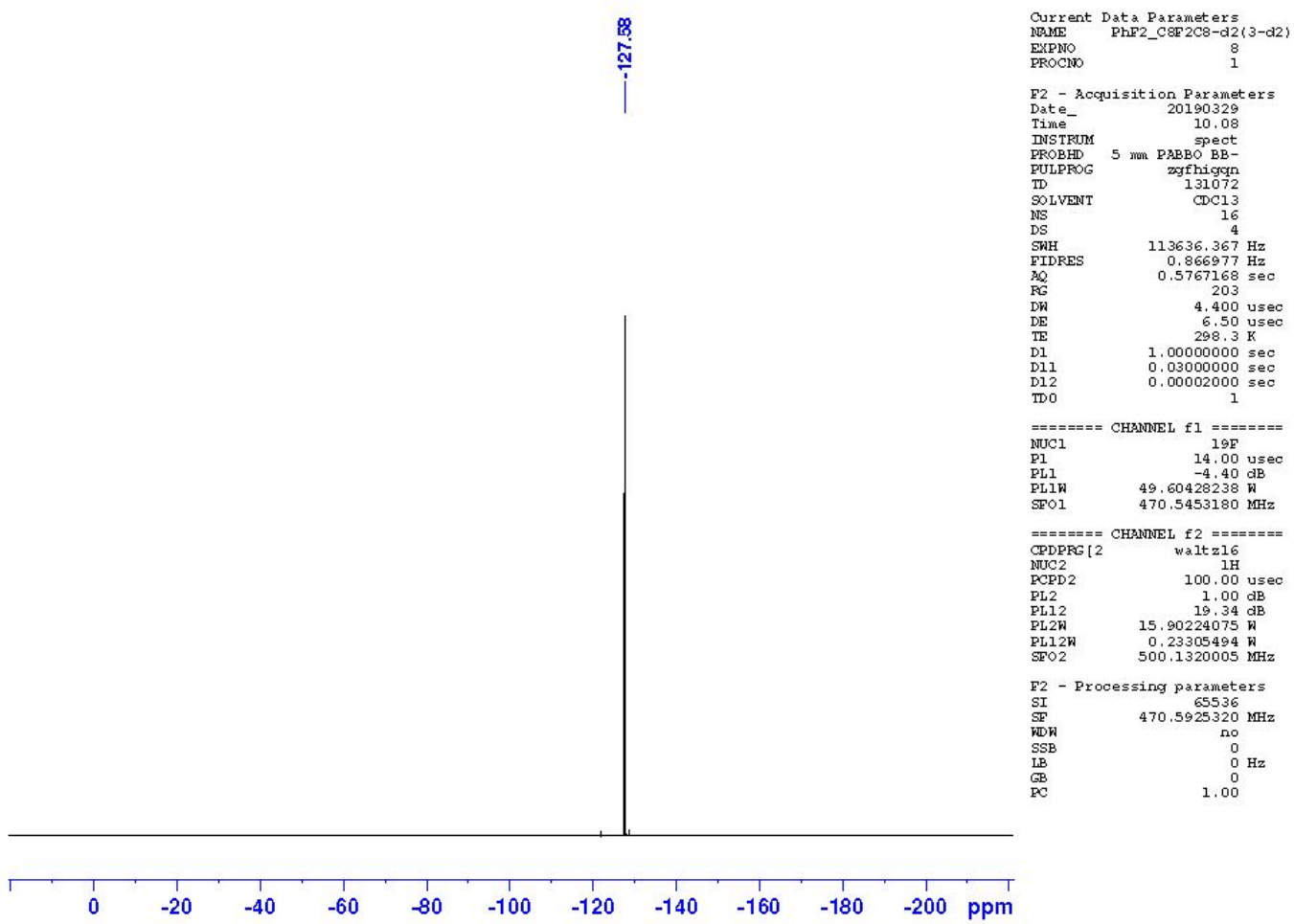

Figure S34. ${ }^{19} \mathrm{~F}$ NMR (471 MHz) spectrum of 1,4-bis(tris-7-octenylsilyl)-4,5-diduterio-2,3difluorobenzene $\left(\mathbf{1}-d_{2}\right)$ in $\mathrm{CDCl}_{3}$. 


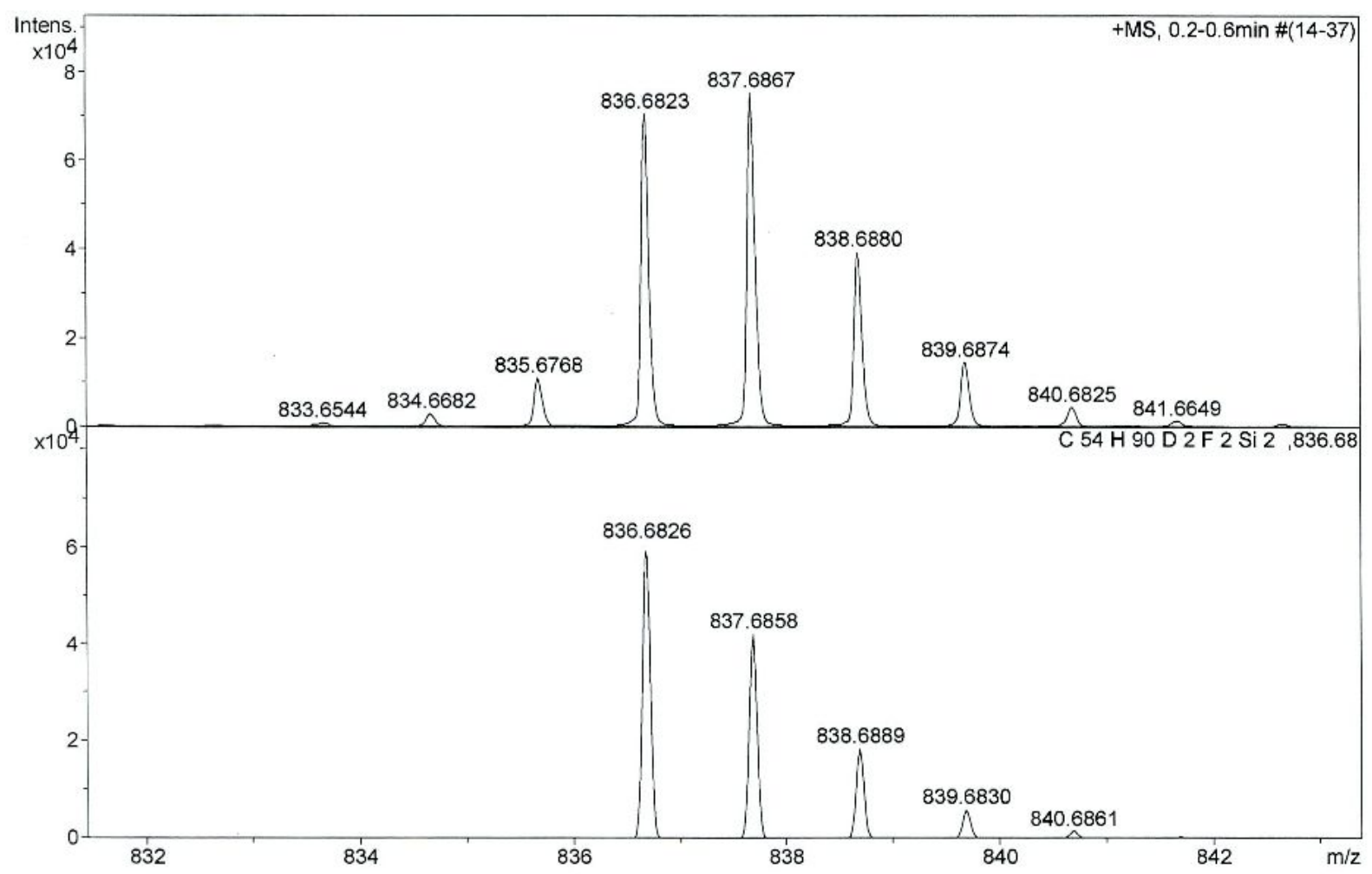

Figure S35. HRMS spectrum of 1,4-bis(tris-7-octenylsilyl)-4,5-diduterio-2,3-difluorobenzene $\left(\mathbf{1}-d_{2}\right)$ (ESI, positive). Top: obsd. Bottom: sim.

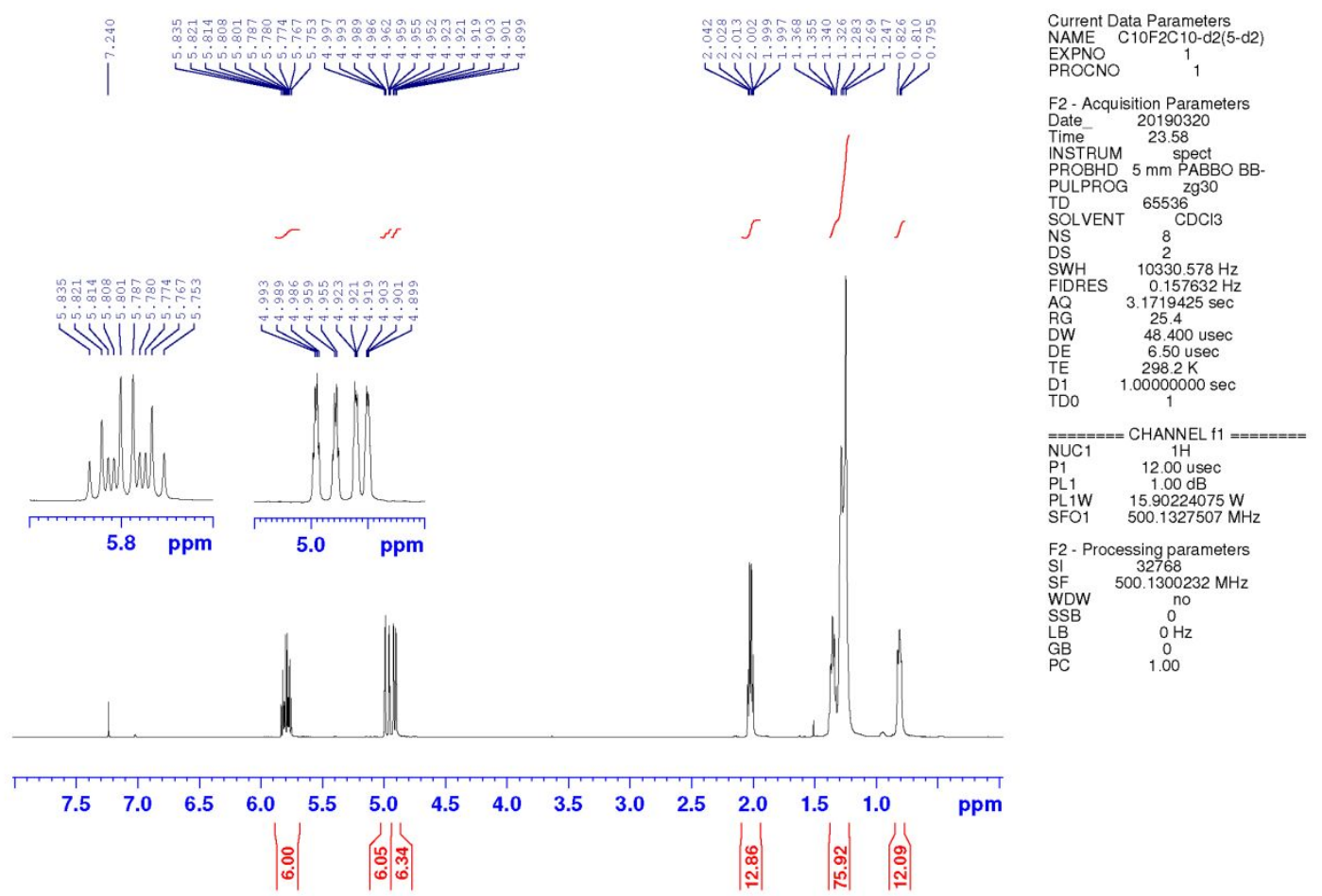

Figure S36. ${ }^{1} \mathrm{H}$ NMR (500 MHz) spectrum of 1,4-bis(tris-9-decenylsilyl)-4,5-diduterio-2,3difluorobenzene $\left(\mathbf{2}-d_{2}\right)$ in $\mathrm{CDCl}_{3}$. 


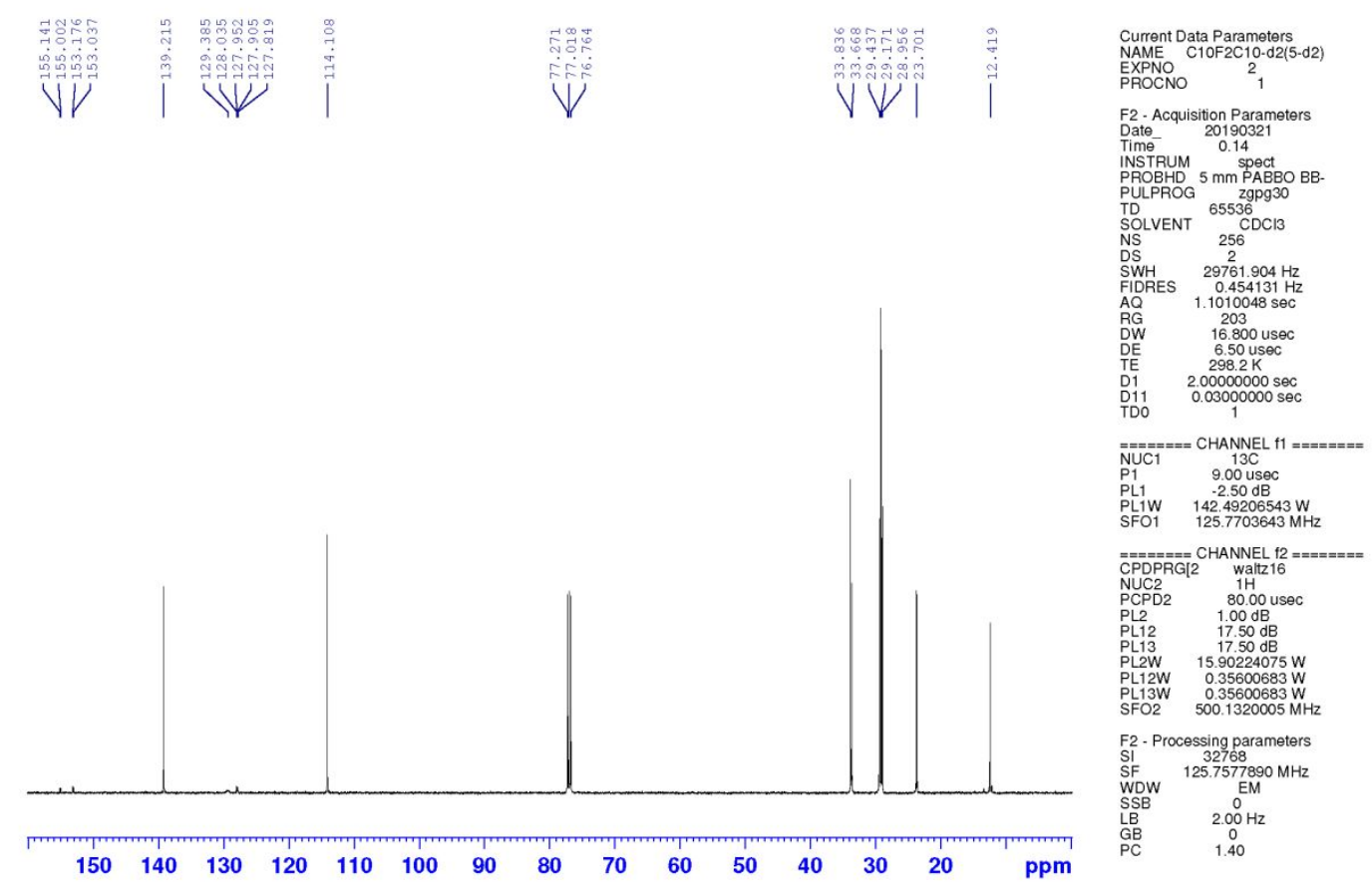

Figure S37. ${ }^{13} \mathrm{C}$ NMR (126 MHz) spectrum of 1,4-bis(tris-9-decenylsilyl)-4,5-diduterio-2,3difluorobenzene $\left(\mathbf{2}-d_{2}\right)$ in $\mathrm{CDCl}_{3}$.

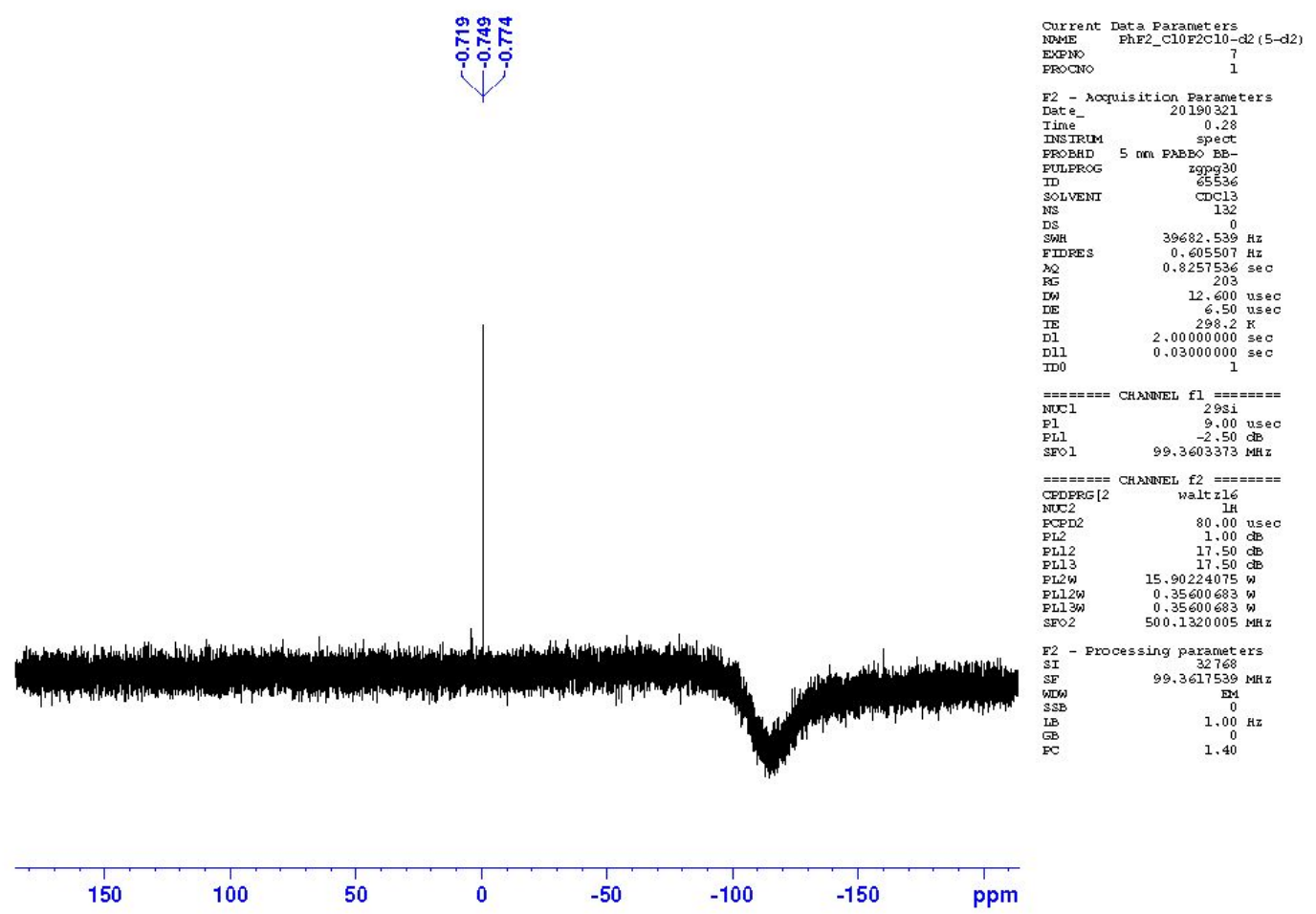

Figure S38. ${ }^{29} \mathrm{Si}$ NMR (99 MHz) spectrum of 1,4-bis(tris-9-decenylsilyl)-4,5-diduterio-2,3difluorobenzene $\left(2-d_{2}\right)$ in $\mathrm{CDCl}_{3}$. 


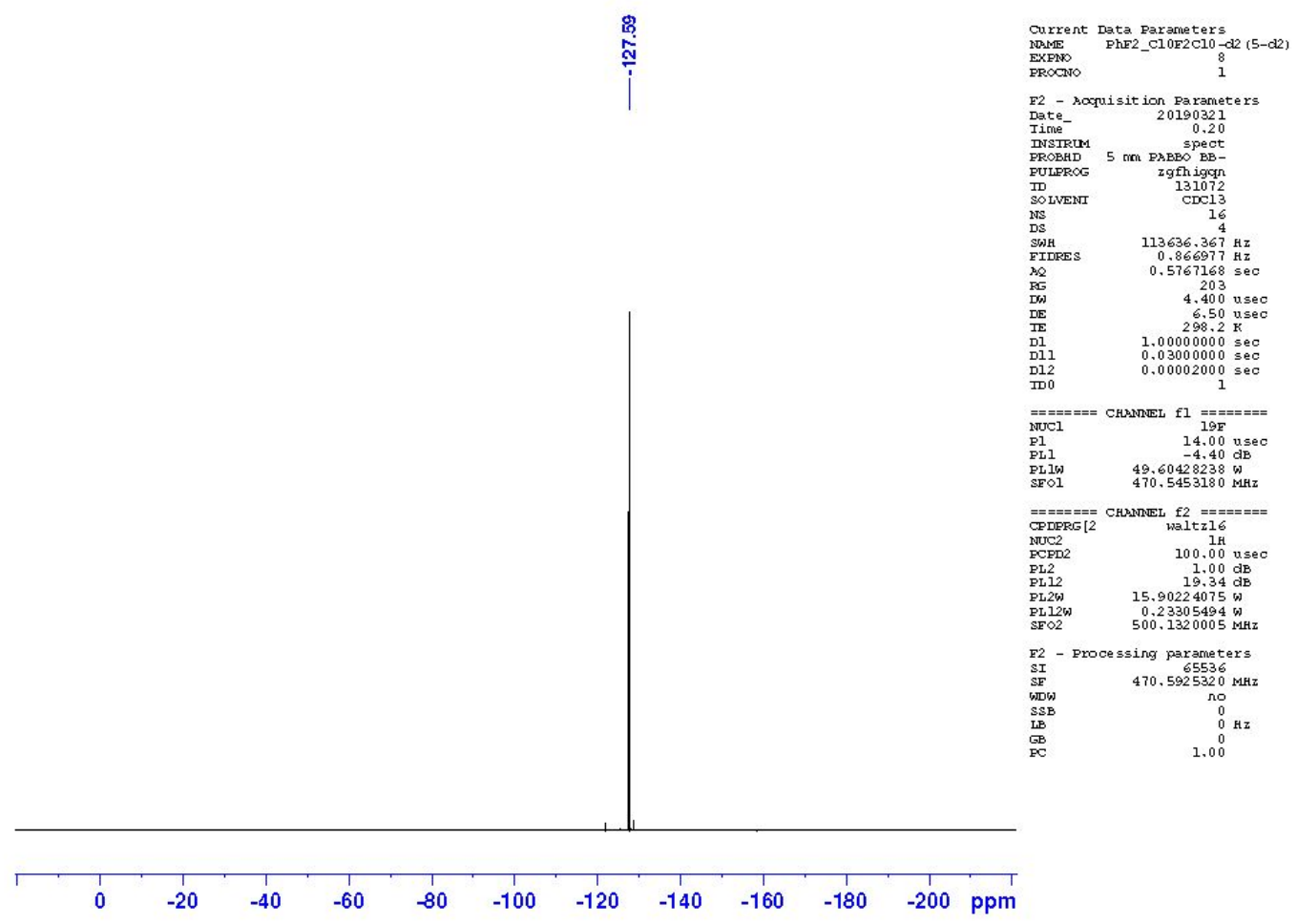

Figure S39. ${ }^{19} \mathrm{~F}$ NMR (471 MHz) spectrum of 1,4-bis(tris-9-decenylsilyl)-4,5-diduterio-2,3difluorobenzene $\left(2-d_{2}\right)$ in $\mathrm{CDCl}_{3}$.

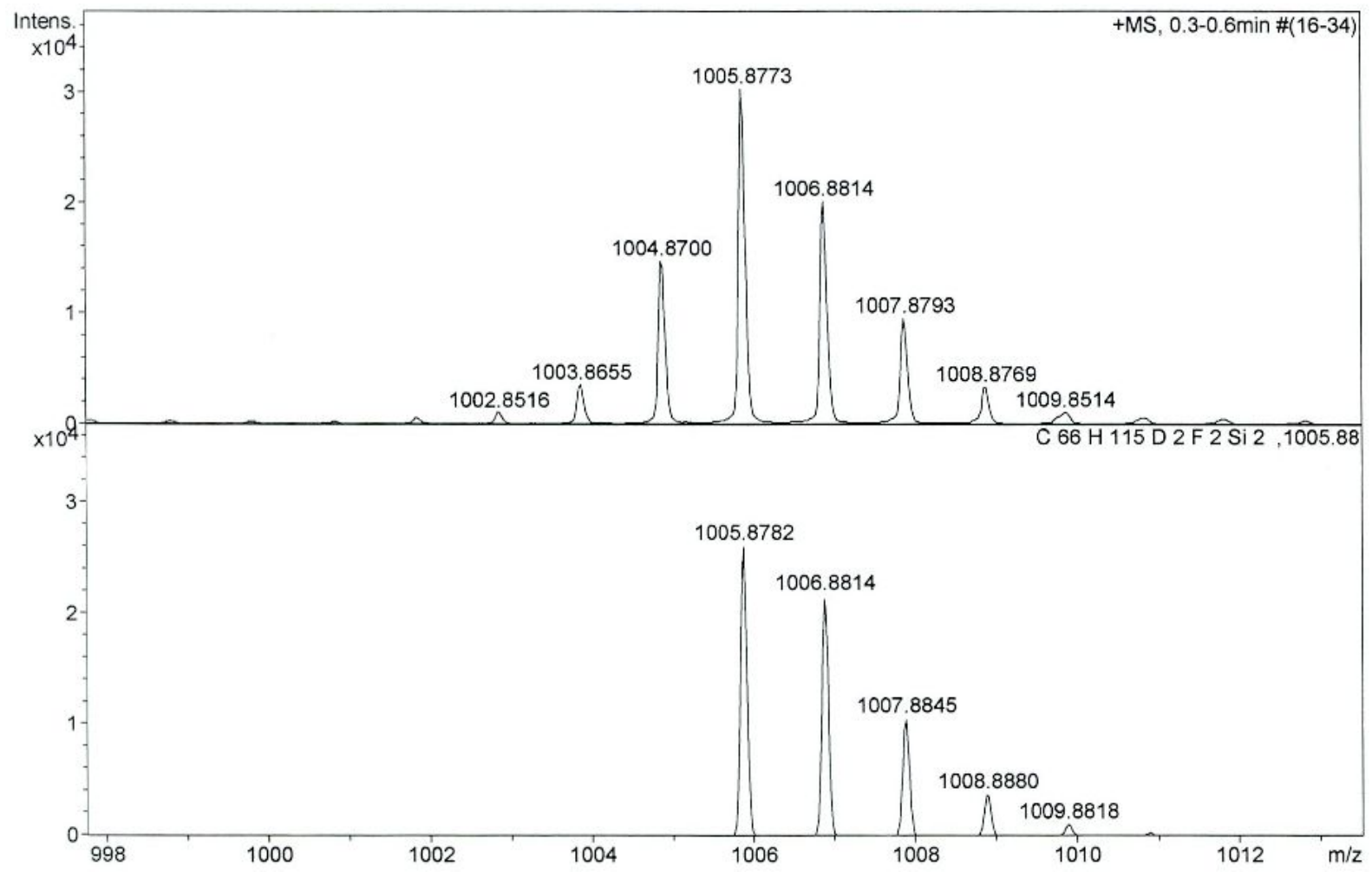

Figure S40. HRMS spectrum of 1,4-bis(tris-9-decenylsilyl)-4,5-diduterio-2,3-difluorobenzene $\left(\mathbf{2}-d_{2}\right)$ (ESI, positive). Top: obsd. Bottom: sim. 


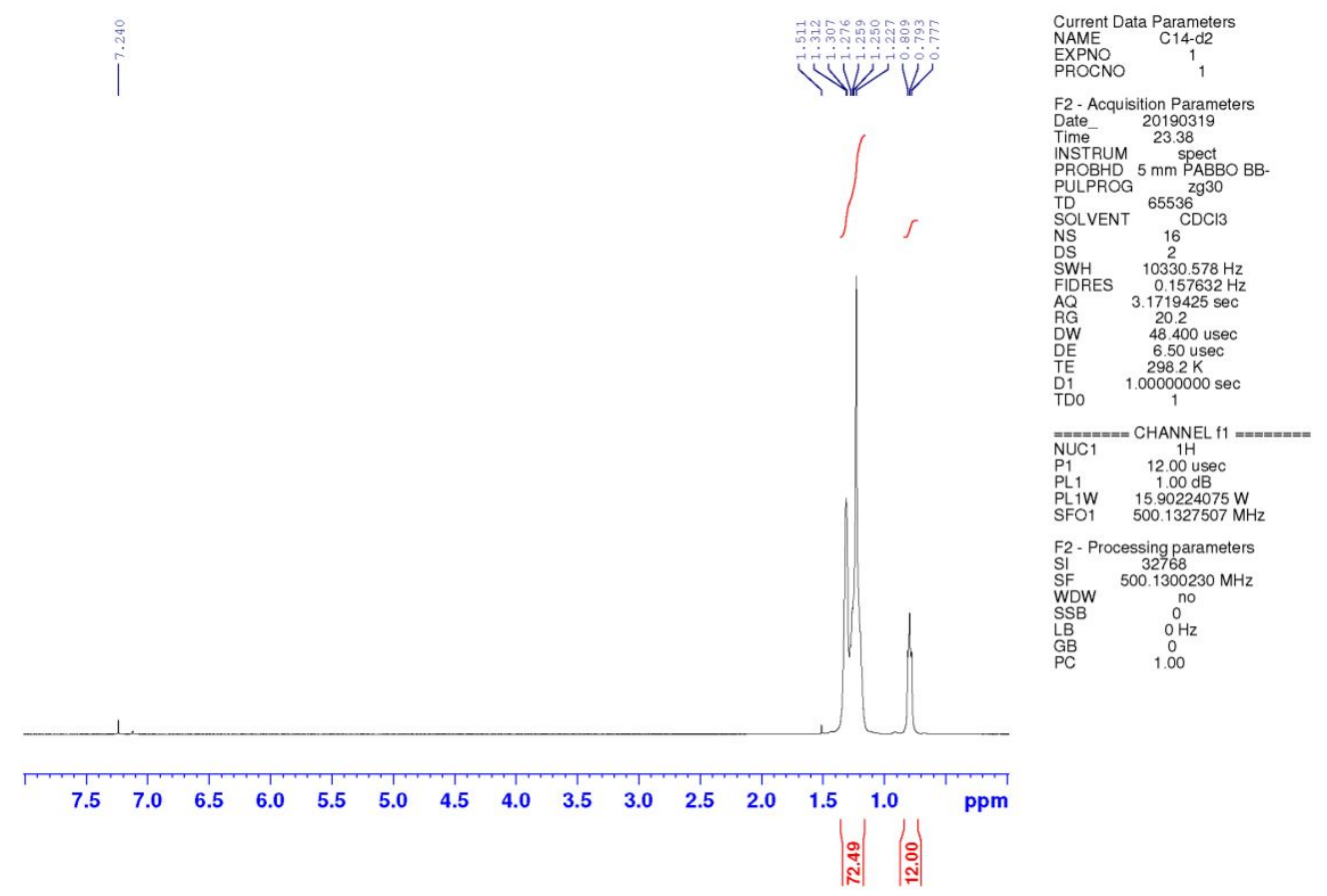

Figure S41. ${ }^{1} \mathrm{H}$ NMR $(500 \mathrm{MHz})$ spectrum of deuterated molecular gyrotop C14PhF2- $d_{2}$ in $\mathrm{CDCl}_{3}$.

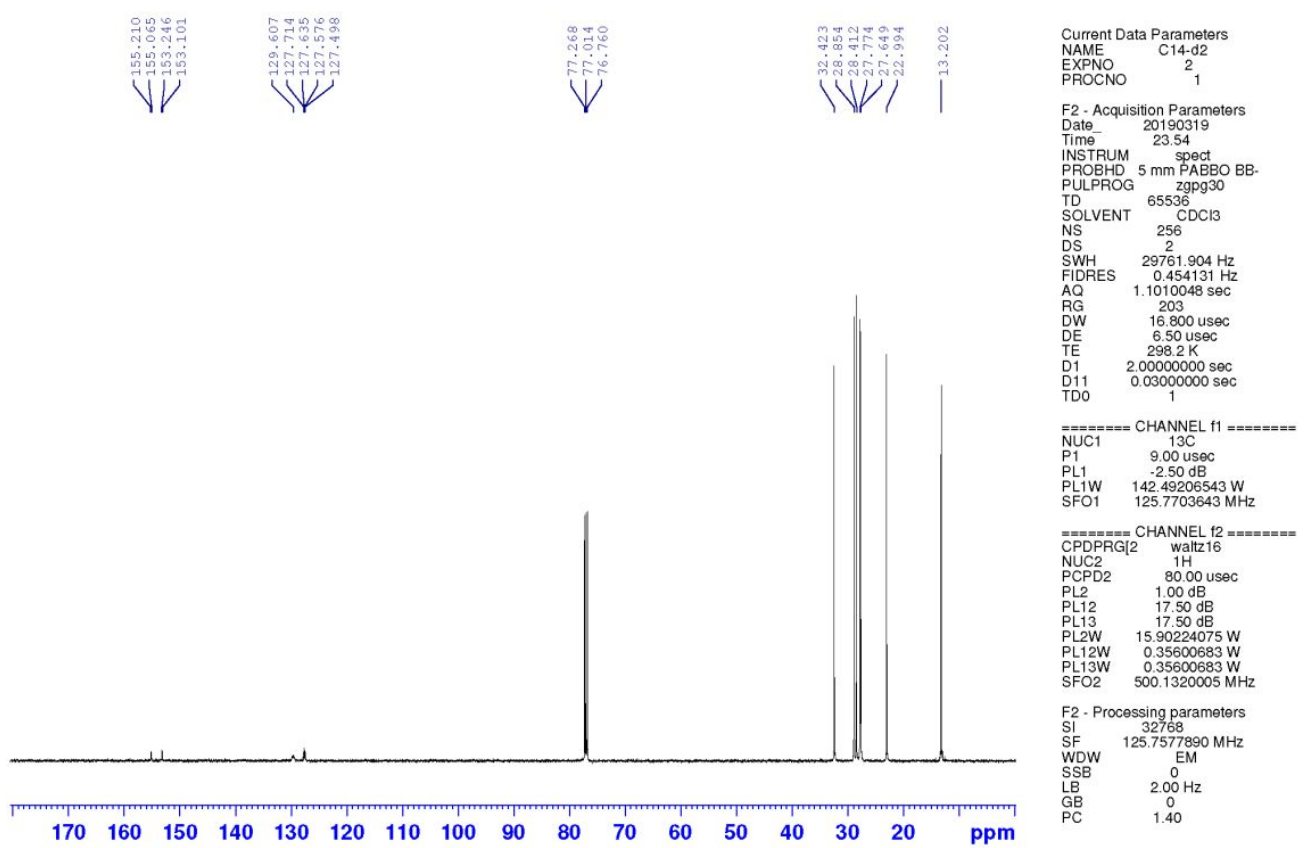

Figure S42. ${ }^{13} \mathrm{C}$ NMR (126 MHz) spectrum of deuterated molecular gyrotop C14PhF2- $d_{2}$ in $\mathrm{CDCl}_{3}$. 


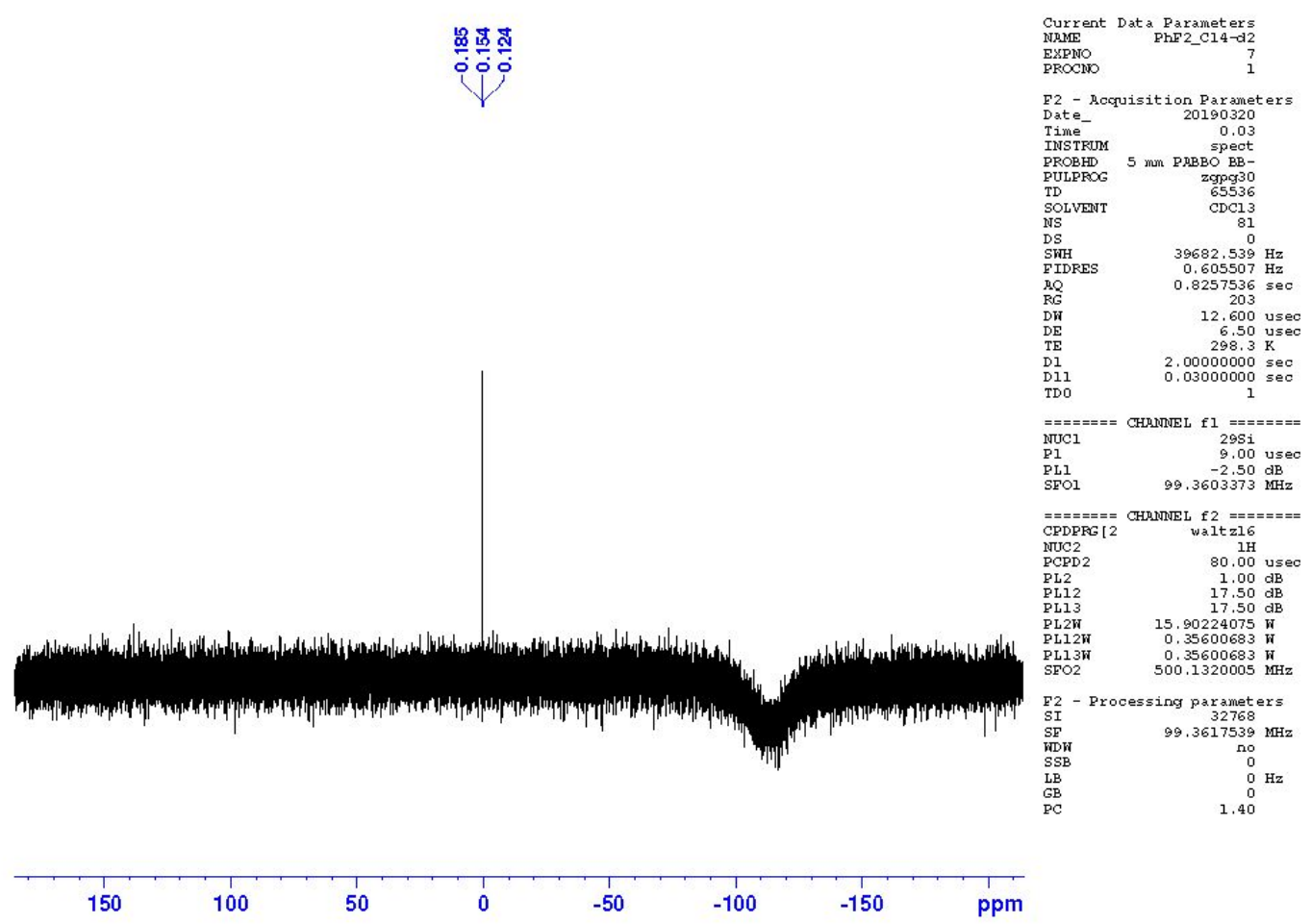

Figure S43. ${ }^{29} \mathrm{Si}$ NMR (99 MHz) spectrum of deuterated molecular gyrotop C14PhF2- $d_{2}$ in $\mathrm{CDCl}_{3}$.

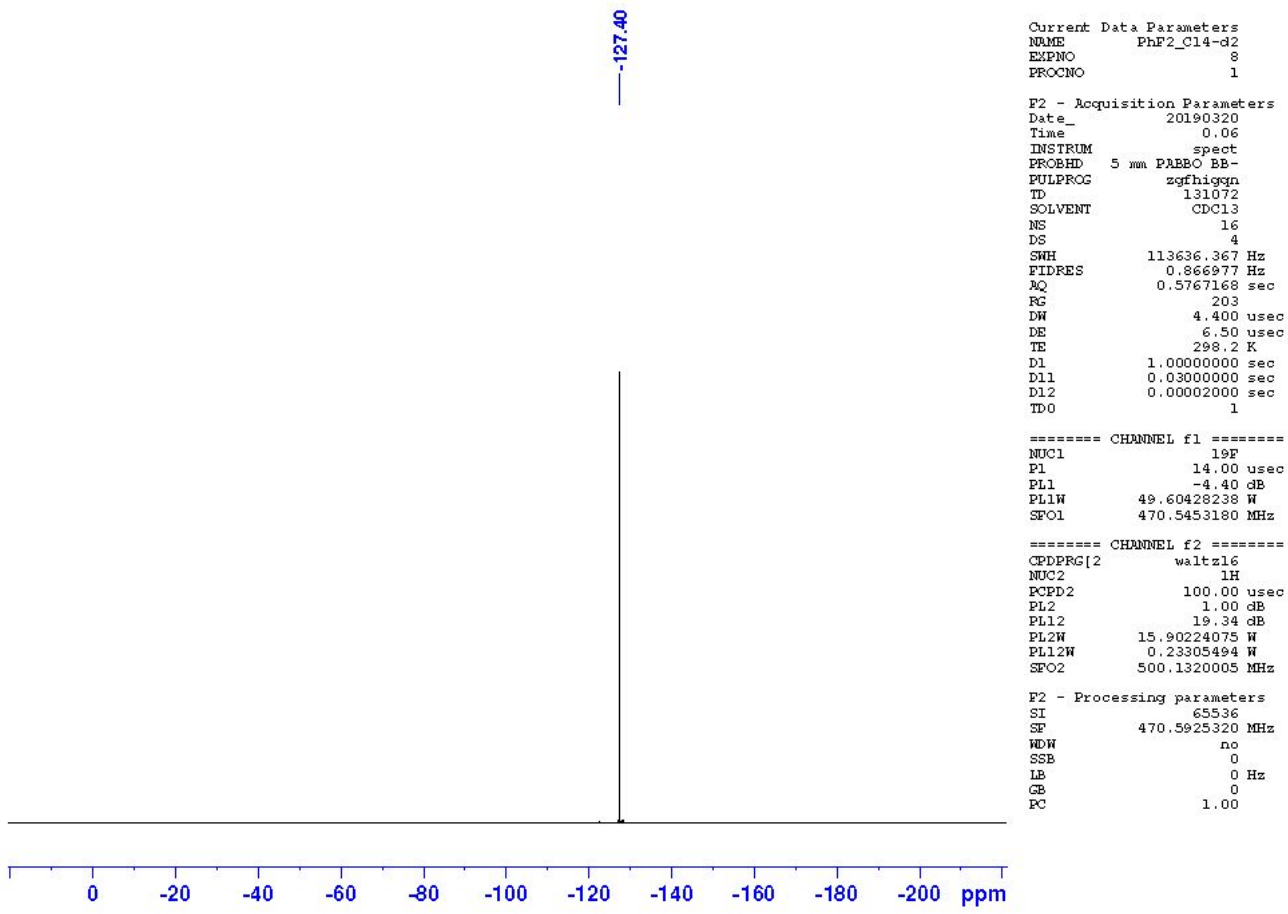

Figure S44. ${ }^{19} \mathrm{~F}$ NMR (471 MHz) spectrum of deuterated molecular gyrotop C14PhF2- $d_{2}$ in $\mathrm{CDCl}_{3}$. 


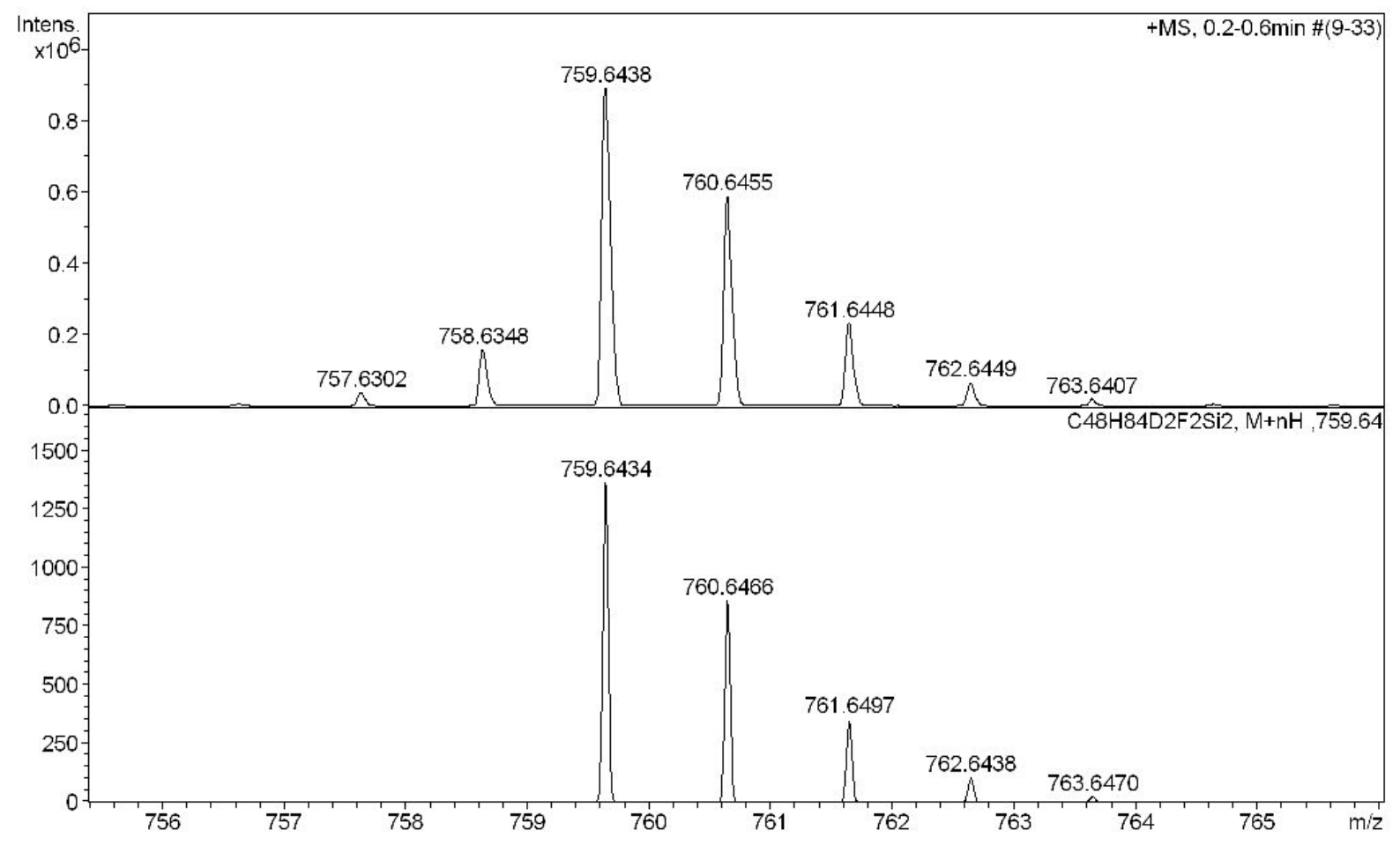

Figure S45. HRMS spectrum of deuterated molecular gyrotop C14PhF2- $d_{2}$ (ESI, positive). Top: obsd. Bottom: sim.

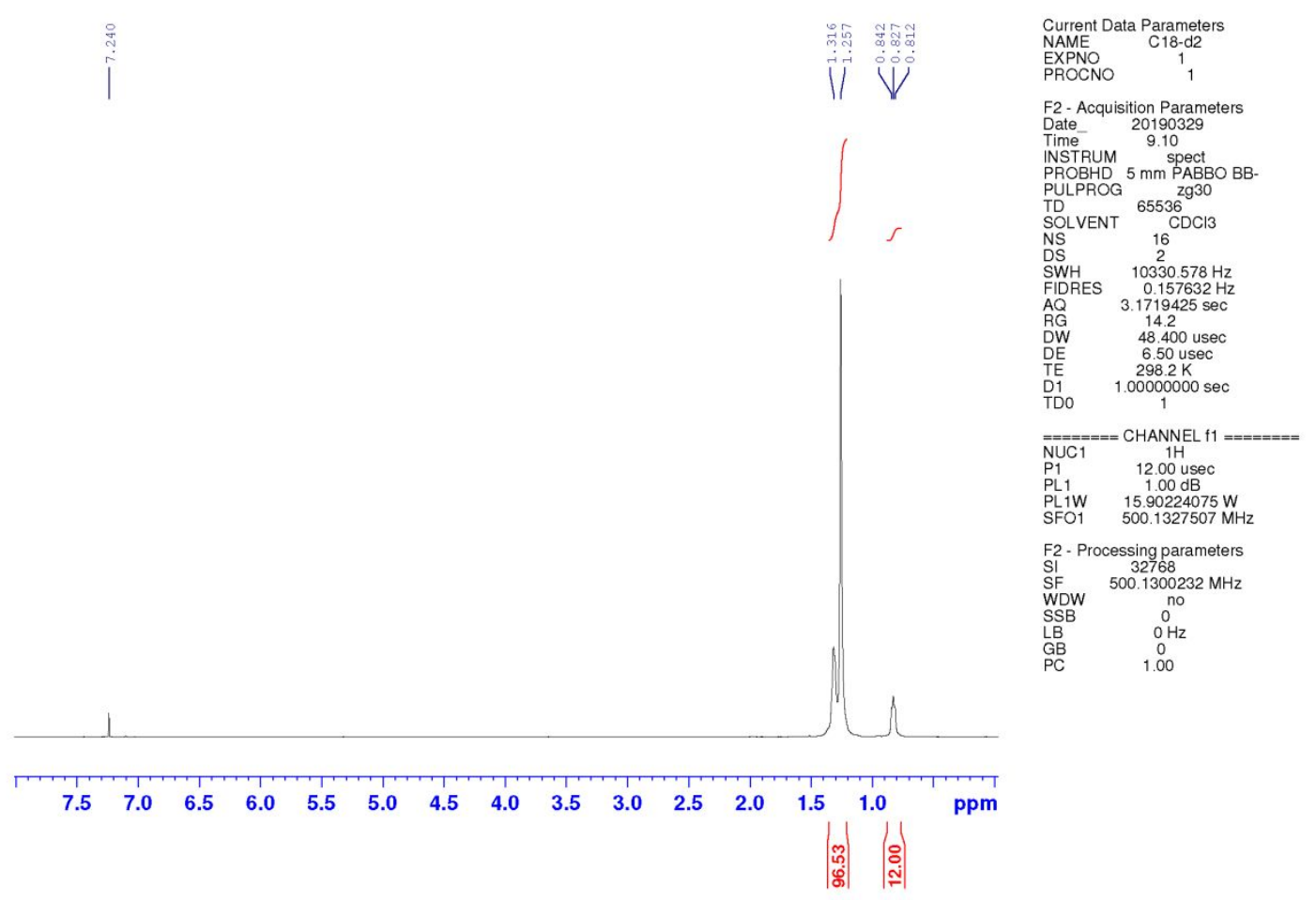

Figure S46. ${ }^{1} \mathrm{H}$ NMR $(500 \mathrm{MHz})$ spectrum of deuterated molecular gyrotop C18PhF2- $d_{2}$ in $\mathrm{CDCl}_{3}$. 


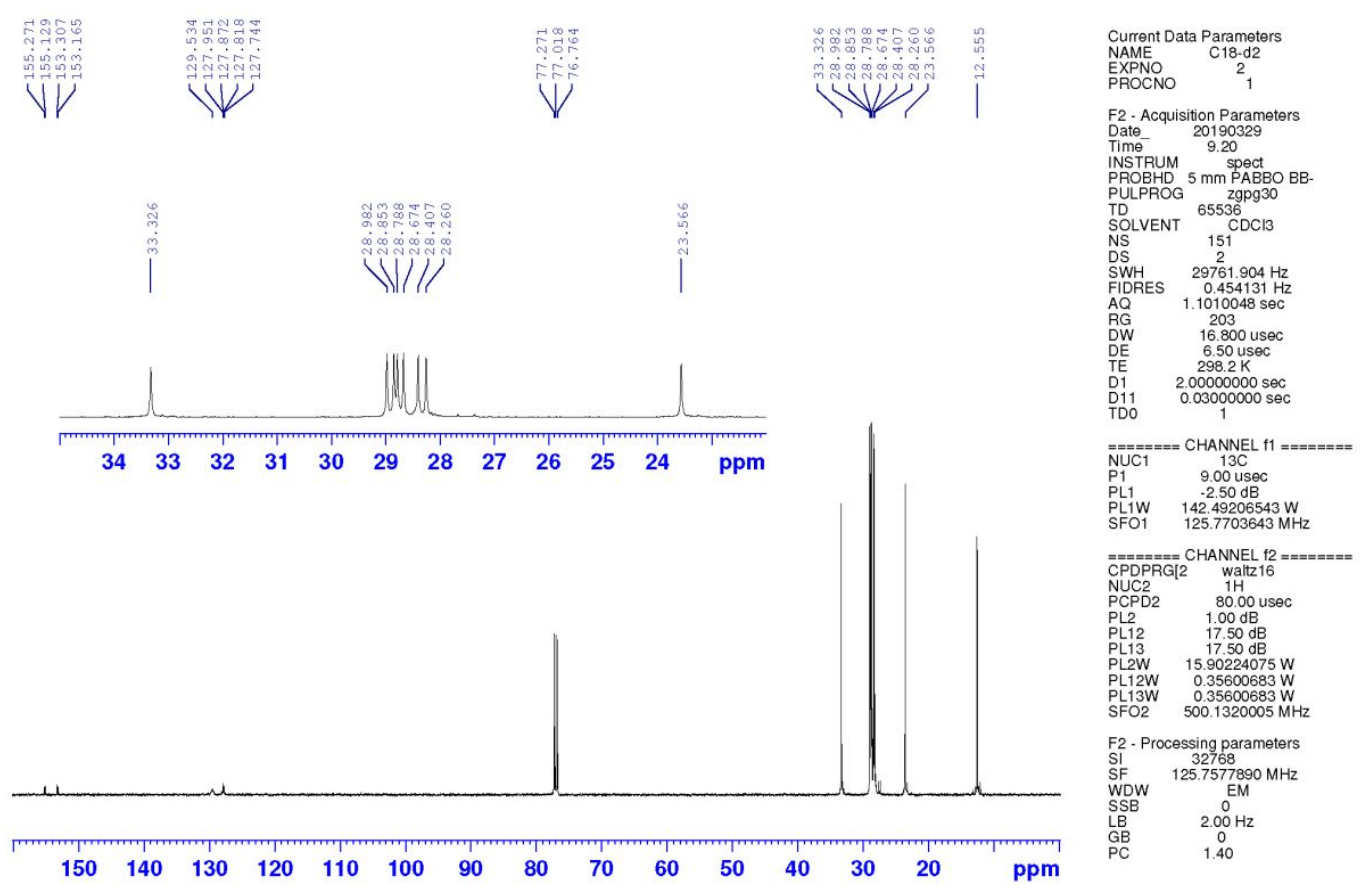

Figure S47. ${ }^{13} \mathrm{C}$ NMR (126 MHz) spectrum of deuterated molecular gyrotop C18PhF2- $d_{2}$ in $\mathrm{CDCl}_{3}$.

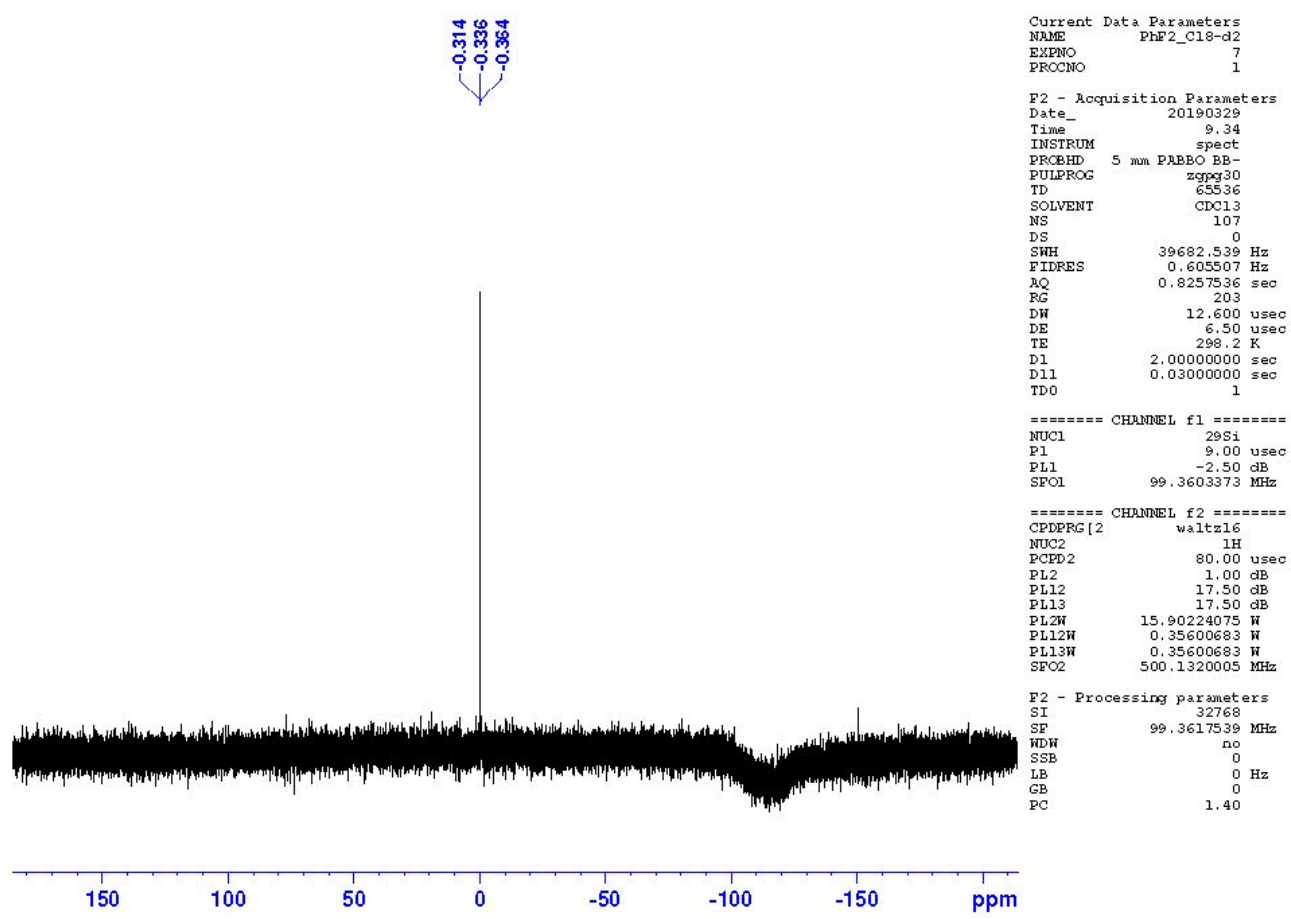

Figure S48. ${ }^{29} \mathrm{Si}$ NMR (99 MHz) spectrum of deuterated molecular gyrotop C18PhF2- $d_{2}$ in $\mathrm{CDCl}_{3}$. 


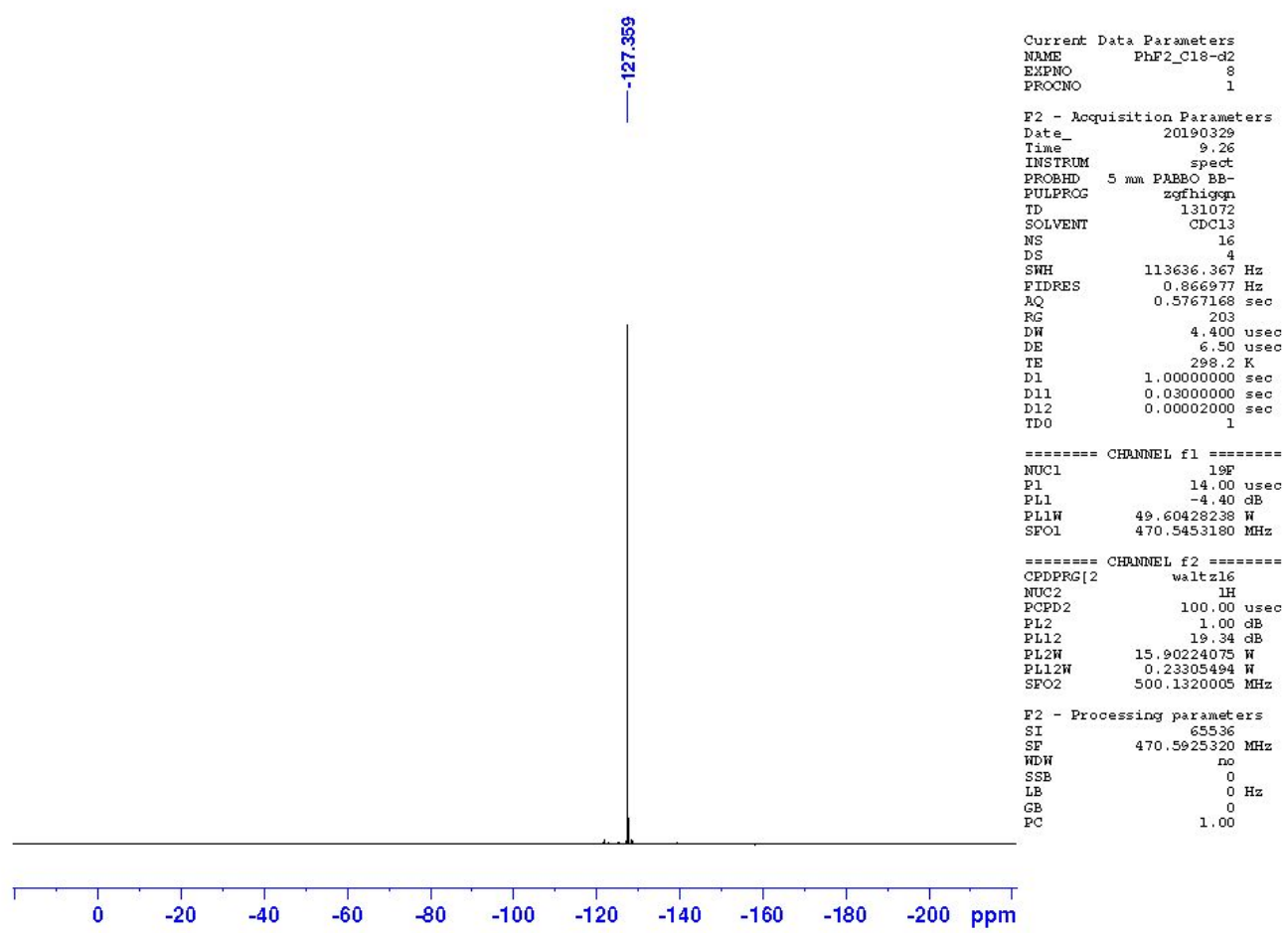

Figure S49. ${ }^{19} \mathrm{~F}$ NMR (471 MHz) spectrum of deuterated molecular gyrotop C18PhF2- $d_{2}$ in $\mathrm{CDCl}_{3}$.

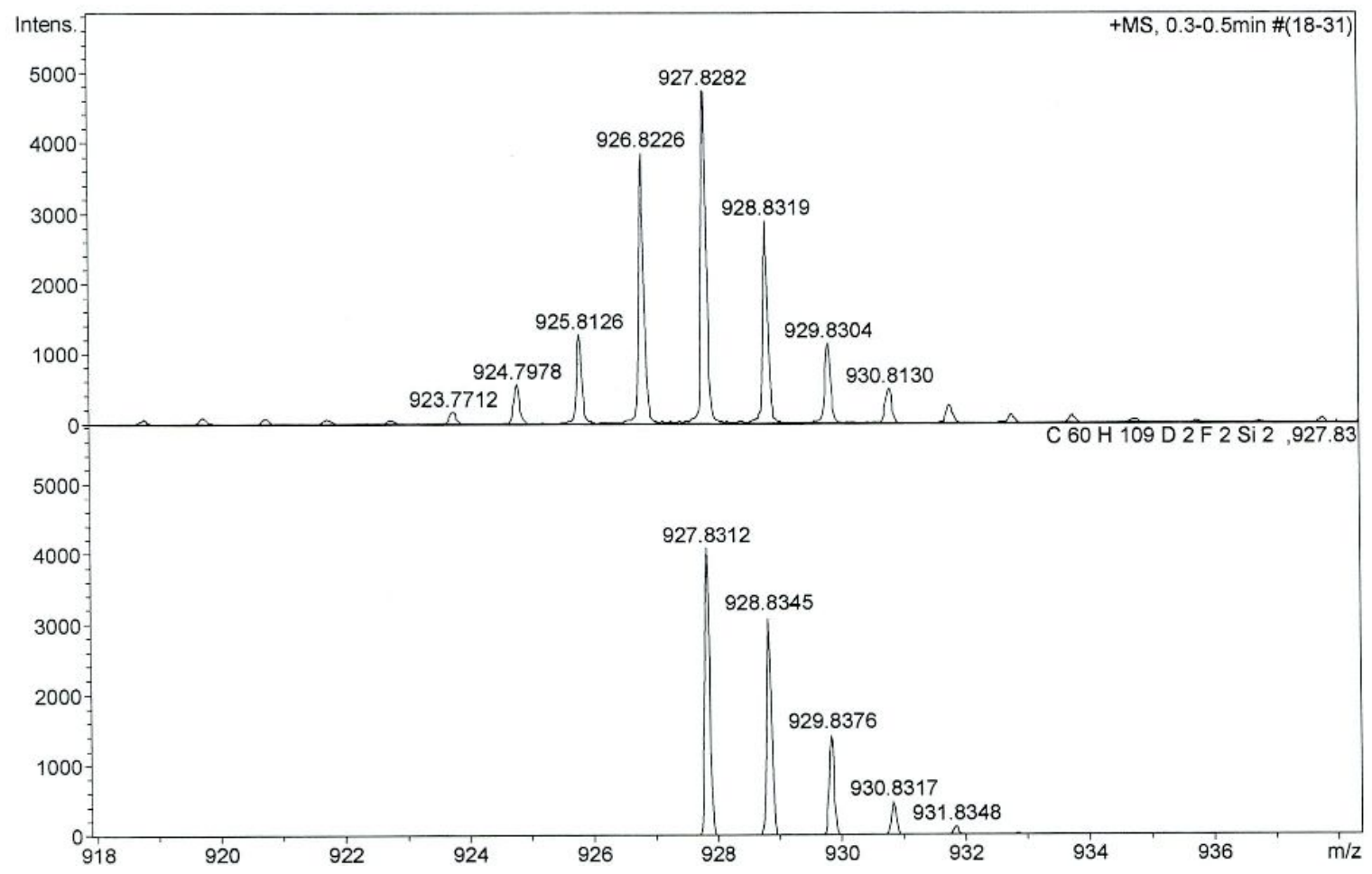

Figure S50. HRMS spectrum of deuterated molecular gyrotop C18PhF2- $d_{2}$ (ESI, positive). Top: obsd. Bottom: sim. 


\section{Details of X-Ray Diffraction Studies}

\section{a. Crystal Data}

Table S1. Crystal Data

\begin{tabular}{|c|c|c|c|}
\hline \multicolumn{2}{|l|}{ Compound } & C14PhF2 & $\mathrm{C} 18 \mathrm{PhF} 2$ \\
\hline \multicolumn{2}{|l|}{ CCDC \# } & 2022211 & 2022212 \\
\hline \multicolumn{2}{|l|}{ Temperature } & $200 \mathrm{~K}$ & $200 \mathrm{~K}$ \\
\hline \multicolumn{2}{|l|}{ Empirical formula } & C48 H86 F2 Si2 & C60 H110 F2 Si2 \\
\hline \multicolumn{2}{|l|}{ Crystal shape } & prism & prism \\
\hline \multicolumn{2}{|l|}{ Crystal color } & colorless & colorless \\
\hline \multicolumn{2}{|l|}{ Crystal size } & $0.300 \times 0.300 \times 0.180 \mathrm{~mm}^{3}$ & $0.489 \times 0.464 \times 0.064 \mathrm{~mm}^{3}$ \\
\hline \multicolumn{2}{|l|}{ Formula weight / $\mathrm{g} \mathrm{mol}^{-1}$} & 757.34 & 925.65 \\
\hline \multicolumn{2}{|l|}{ Crystal system } & Monoclinic & Triclinic \\
\hline \multicolumn{2}{|l|}{ Space group } & $\mathrm{C} 2 / \mathrm{c}$ & $P-1$ \\
\hline \multicolumn{2}{|l|}{$\mathbf{Z}$} & 4 & 2 \\
\hline \multicolumn{2}{|l|}{ Calculated density } & $1.032 \mathrm{Mg} / \mathrm{m}^{3}$ & $1.016 \mathrm{Mg} / \mathrm{m}^{3}$ \\
\hline \multirow{7}{*}{ Cell parameter } & $a$ & $25.2149(8) \AA$ & $12.3088(18) \AA$ \\
\hline & $b$ & $11.9602(4) \AA$ & $13.4038(19) \AA$ \\
\hline & $c$ & $18.5920(6) \AA$ & $18.856(3) \AA$ \\
\hline & $\alpha$ & $90^{\circ}$ & $77.061(2)^{\circ}$ \\
\hline & $\beta$ & $119.6630(10)^{\circ}$ & $88.517(2)^{\circ}$ \\
\hline & $\gamma$ & $90^{\circ}$ & $86.849(2)^{\circ}$ \\
\hline & $V$ & $4872.1(3) \AA^{3}$ & $3027.1(8) \AA^{3}$ \\
\hline \multicolumn{2}{|l|}{$\mathbf{F}(000)$} & 1680 & 1032 \\
\hline \multicolumn{2}{|l|}{ Absorption coefficient } & $0.928 \mathrm{~mm}^{-1}$ & $0.098 \mathrm{~mm}^{-1}$ \\
\hline \multicolumn{2}{|l|}{$\begin{array}{c}\theta \text { range for collection } \\
\text { (deg) }\end{array}$} & 4.035 to $71.298^{\circ}(\mathrm{Cu})$ & 1.561 to $27.330^{\circ}(\mathrm{Mo})$ \\
\hline \multicolumn{2}{|l|}{ Index ranges } & $\begin{array}{c}-30<=\mathrm{h}<=29,-13<=\mathrm{k}<=12,- \\
22<=\mathrm{l}<=21\end{array}$ & $\begin{array}{c}-15<=\mathrm{h}<=13,-16<=\mathrm{k}<=17,- \\
23<=\mathrm{l}<=21\end{array}$ \\
\hline \multicolumn{2}{|l|}{ Reflections collected } & 22514 & 15362 \\
\hline \multicolumn{2}{|l|}{$\begin{array}{l}\text { Independent } \\
\text { reflections }\end{array}$} & $4444[\mathrm{R}($ int $)=0.0278]$ & $11677[\mathrm{R}(\mathrm{int})=0.0253]$ \\
\hline \multicolumn{2}{|l|}{ Completeness } & $96.1 \%$ & $97.5 \%$ \\
\hline \multicolumn{2}{|l|}{ Goodness-of-fit on $F^{2}$} & 1.454 & 1.043 \\
\hline \multicolumn{2}{|l|}{$\begin{array}{l}\text { Final } R \text { indices } \\
{[\mathbf{I}>2 \text { sigma(I)] }}\end{array}$} & $\mathrm{R} 1=0.1072, \mathrm{wR} 2=0.3439$ & $\mathrm{R} 1=0.0553, \mathrm{wR} 2=0.1411$ \\
\hline \multicolumn{2}{|l|}{$\mathbf{R}$ indices (all data) } & $\mathrm{R} 1=0.1426, \mathrm{wR} 2=0.3845$ & $\mathrm{R} 1=0.0825, \mathrm{wR} 2=0.1621$ \\
\hline \multicolumn{2}{|l|}{$\begin{array}{l}\text { Largest diff. peak and } \\
\text { hole }\end{array}$} & 0.433 and -0.253 e..$\AA^{-3}$ & 0.418 and -0.270 e..$\AA^{-3}$ \\
\hline
\end{tabular}




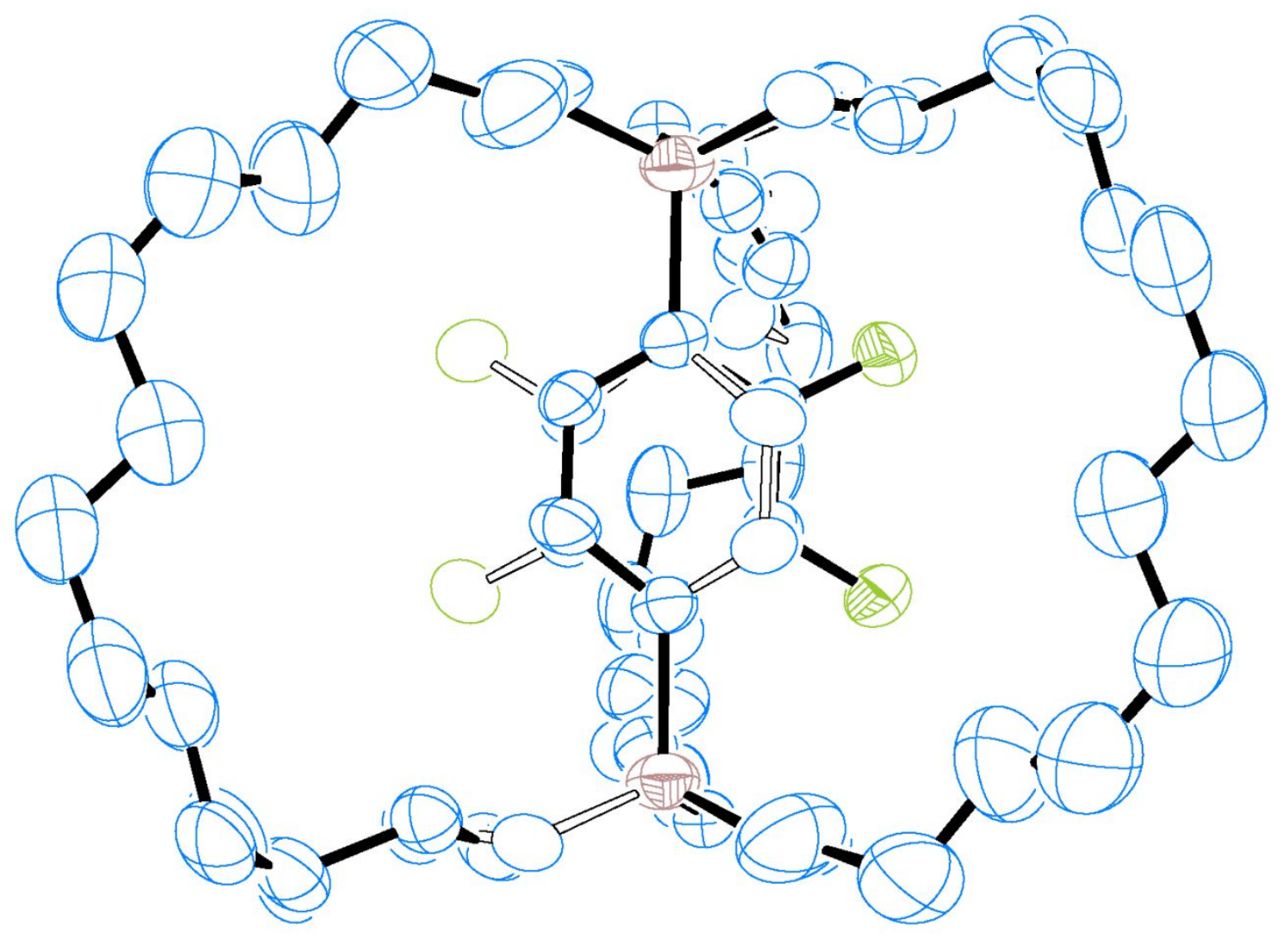

Figure S51. ORTEP drawing of the molecular structure of C14PhF2. Hydrogen atoms are omitted for clarity. (30\% thermal ellipsoids, Disordered atoms are also indicated.) 


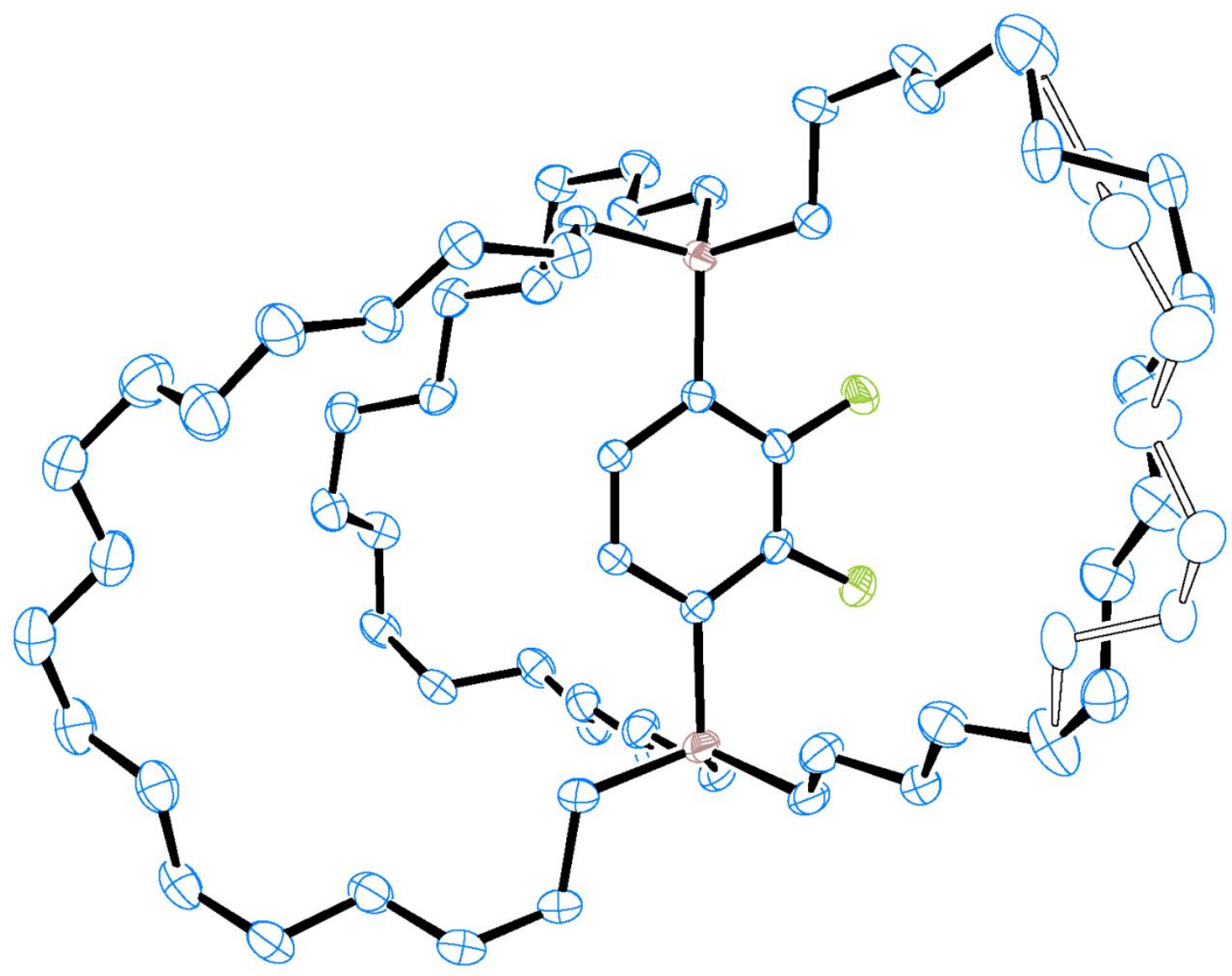

Figure S52. ORTEP drawing of the molecular structure of C18PhF2. Hydrogen atoms are omitted for clarity. (30\% thermal ellipsoids, Disordered atoms are also indicated.) 
(a)

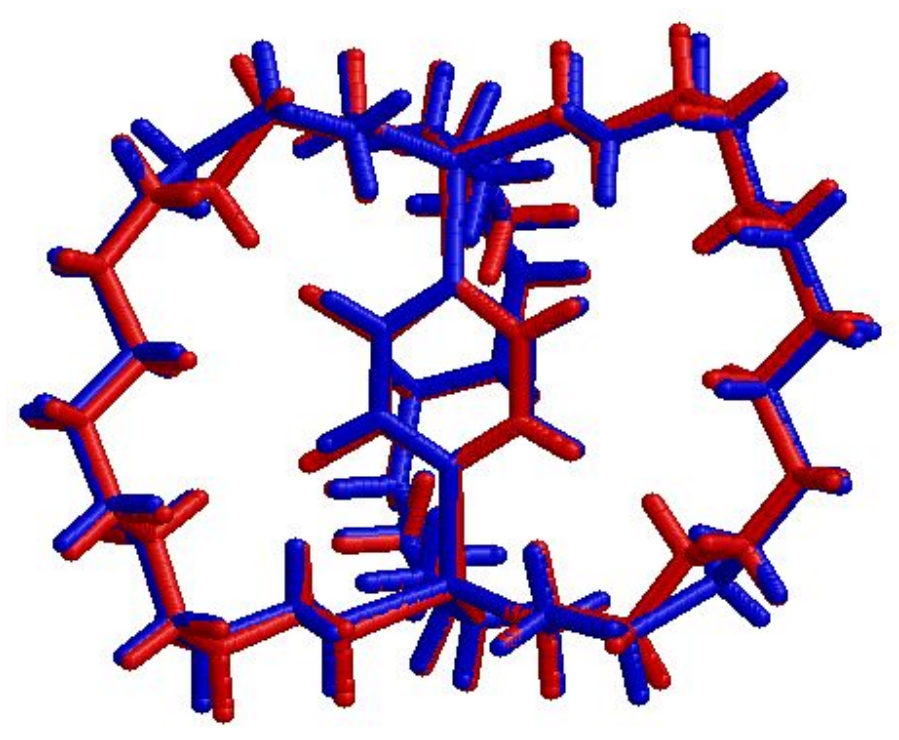

(b)

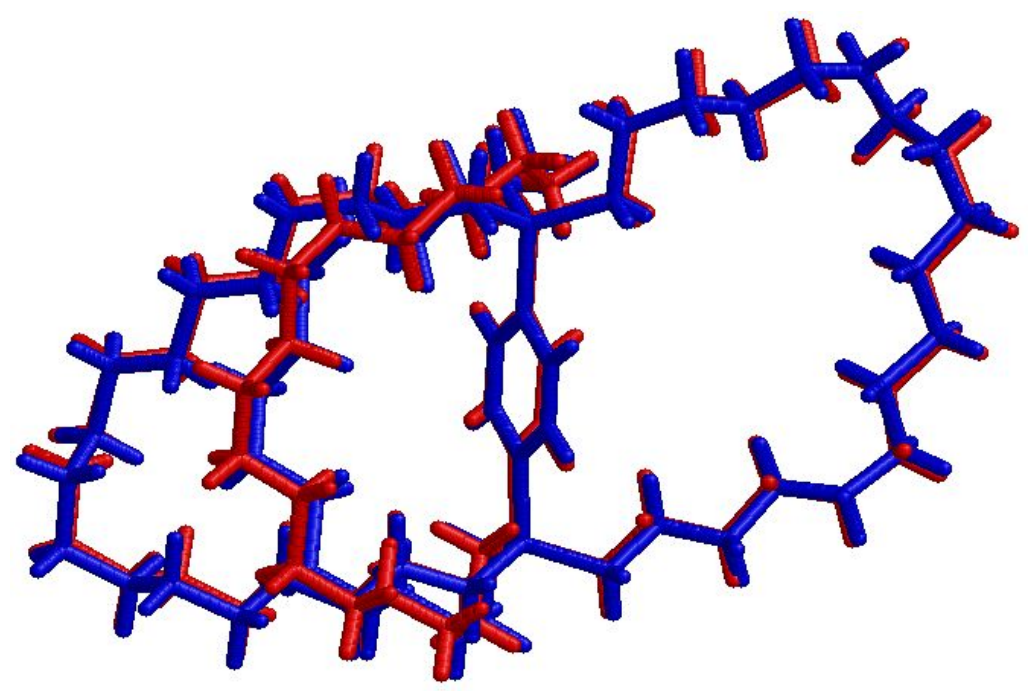

Figure S53. Comparison between the molecular structures between difluorophenylene (red) and phenylene (blue) bridged molecular gyrotops determined by X-ray crystallography: (a) C14PhF2 and C14Ph; (b) C18PhF2 and C18Ph. 
(a)
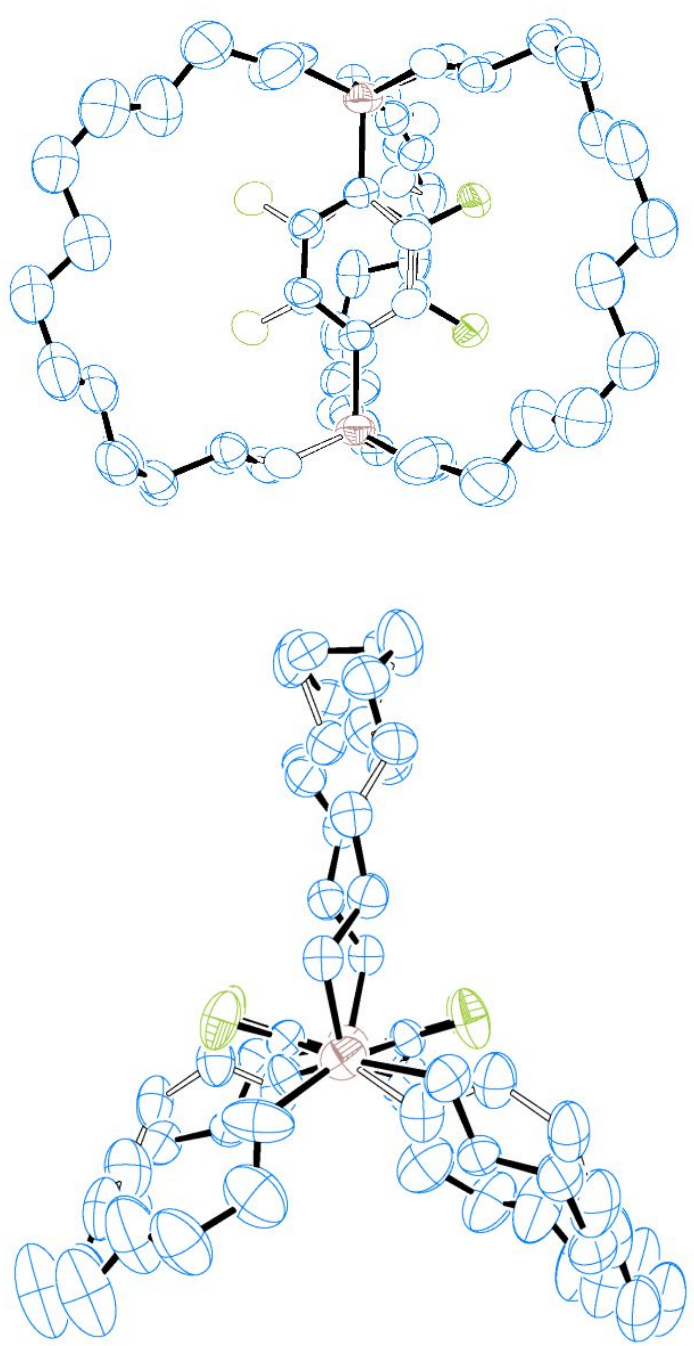

(b)
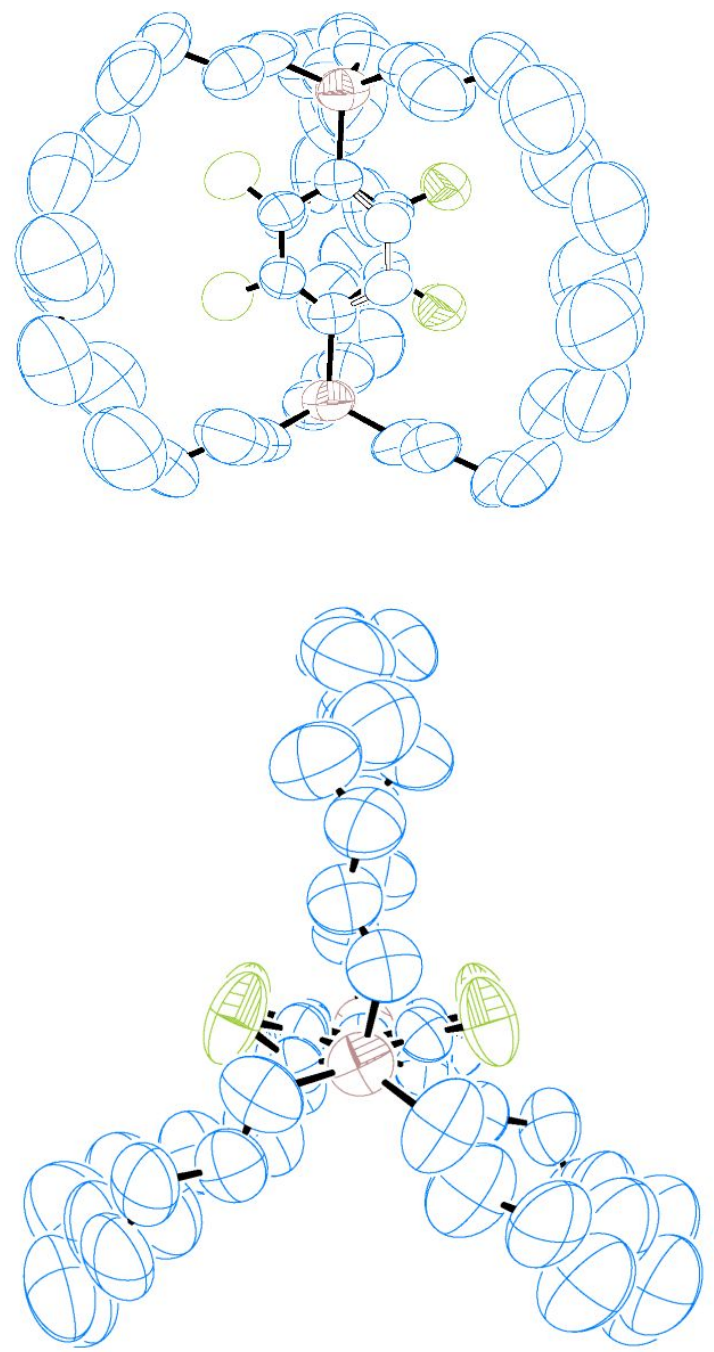

Figure S54. ORTEP drawing (top: equatorial view, bottom: axial view around the difluorophenylene ring) for C14PhF2 at (a) $200 \mathrm{~K}$ (shown in the main text) and (b) $300 \mathrm{~K}$. (30\% thermal ellipsoids, Hydrogen atoms are omitted.) 


\section{Details of Temperature Dependent ${ }^{2} \mathrm{H}$ NMR Spectra}

a. VT-2 ${ }^{2} \mathrm{H} M R$ spectra of C14PhF2- $d_{2}$.
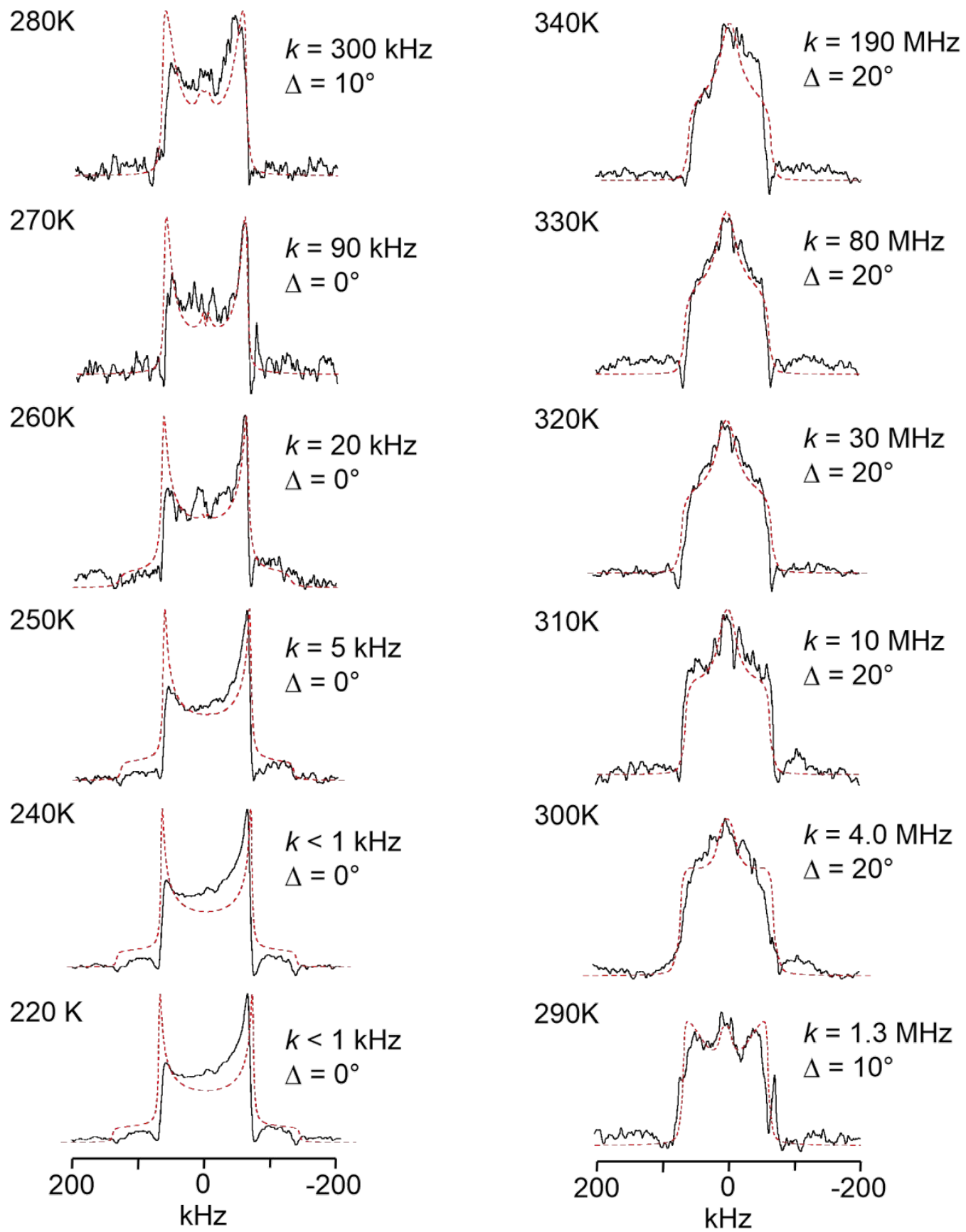

Figure S55. Temperature dependence of solid-state ${ }^{2} \mathrm{H}$ NMR spectra of C14PhF2- $d_{2}$ [solid line: observed spectra; dotted line: spectra simulated with designated exchange rate constants, $k$, and site occupancy factors, $p_{\mathrm{x}}$.]. 


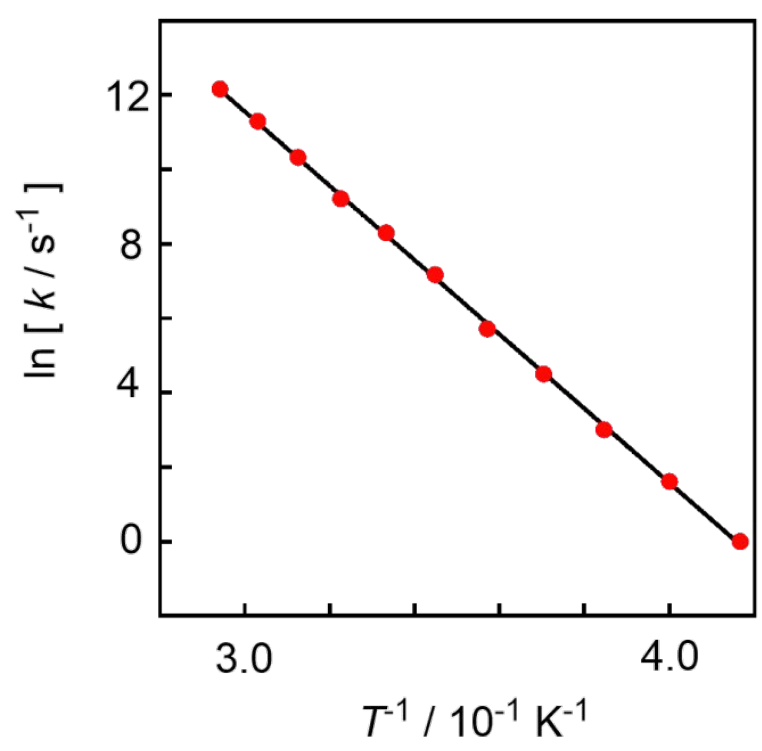

Figure S56. An Arrhenius plots of temperature dependent exchange rates of difluorophenylene flipping determined by $\mathrm{VT}^{2} \mathrm{H}$ NMR. The slope and the intercept of the fitting line are $9978.2 \pm 67.4,41.5 \pm 0.24$, respectively. The activation energy was estimated to be $19.84 \pm 0.13$ $\mathrm{kcal} / \mathrm{mol}$.

b. VT-2 H NMR spectra of C18PhF2- $d_{2}$.

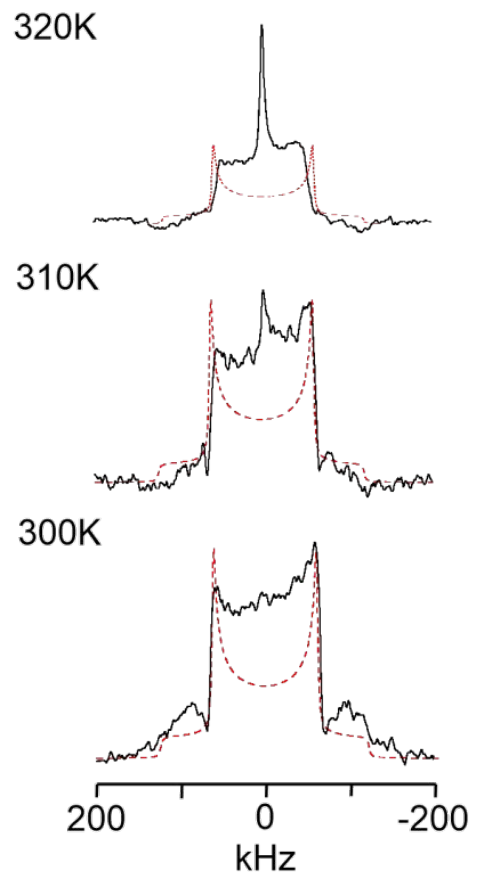

Figure S57. Temperature dependence of solid-state ${ }^{2} \mathrm{H}$ NMR spectra of C18PhF2- $d_{2}$ [solid line: observed spectra; dotted line: spectra simulated by static model]. 


\section{Details of Temperature Dependent Dielectric Relaxation Studies}

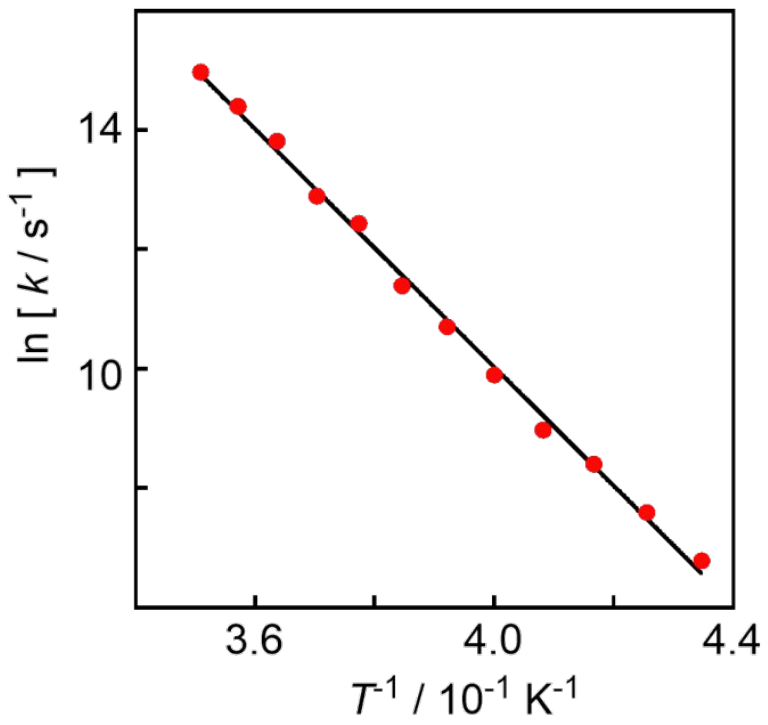

Figure S58. An Arrhenius plot of temperature dependent reciprocal relaxation time determined by VT dielectric relaxation spectra. The slope and the intercept of the fitting line are $9949.9 \pm 171,49.8 \pm 0.67$, respectively. The activation energy was estimated to be $19.78 \pm 0.34$ $\mathrm{kcal} / \mathrm{mol}$. 


\section{Details of DFT Calculations}

All calculations were carried out using Gaussian 16 (Revision C.01) program packages ${ }^{\mathrm{S} 1}$ at the Research Center for Computational Science, Okazaki, Japan

S1: Gaussian 16, Revision C.01,

M. J. Frisch, G. W. Trucks, H. B. Schlegel, G. E. Scuseria, M. A. Robb, J. R. Cheeseman, G. Scalmani, V. Barone, G. A. Petersson, H. Nakatsuji, X. Li, M. Caricato, A. V. Marenich, J. Bloino, B. G. Janesko, R. Gomperts, B. Mennucci, H. P. Hratchian, J. V. Ortiz, A. F. Izmaylov, J. L. Sonnenberg, D. Williams-Young, F. Ding, F. Lipparini, F. Egidi, J. Goings, B. Peng, A. Petrone, T. Henderson, D. Ranasinghe, V. G. Zakrzewski, J. Gao, N. Rega, G. Zheng, W. Liang, M. Hada, M. Ehara, K. Toyota, R. Fukuda, J. Hasegawa, M. Ishida, T. Nakajima, Y. Honda, O. Kitao, H. Nakai, T. Vreven, K. Throssell, J. A. Montgomery, Jr., J. E. Peralta, F. Ogliaro, M. J. Bearpark, J. J. Heyd, E. N. Brothers, K. N. Kudin, V. N. Staroverov, T. A. Keith, R. Kobayashi, J. Normand, K. Raghavachari, A. P. Rendell, J. C. Burant, S. S. Iyengar, J. Tomasi, M. Cossi, J. M. Millam, M. Klene, C. Adamo, R. Cammi, J. W. Ochterski, R. L. Martin, K. Morokuma, O. Farkas, J. B. Foresman, and D. J. Fox, Gaussian, Inc., Wallingford CT, 2019.

Table S2. Optimized Structural Coordinate and its Total Energy for C14PhF2 at B3LYP/6-31G(d) level

\begin{tabular}{|c|c|c|c|c|c|}
\hline \multirow{3}{*}{$\begin{array}{l}\text { Center } \\
\text { Number } \\
1\end{array}$} & \multirow{3}{*}{$\begin{array}{c}\text { Atomic } \\
\text { Number } \\
14\end{array}$} & \multirow{2}{*}{$\begin{array}{l}\text { Atomic } \\
\text { Type }\end{array}$} & \multicolumn{3}{|c|}{ Coordinates (Angstroms) } \\
\hline & & & $\mathrm{X}$ & $\mathrm{Y}$ & Z \\
\hline & & 0 & -0.174487 & -1.571829 & -2.952946 \\
\hline 2 & 14 & 0 & -0.184681 & 1.404959 & 3.057531 \\
\hline 3 & 6 & 0 & -1.966637 & -1.331742 & -3.554462 \\
\hline 4 & 6 & 0 & -1.955614 & 1.062374 & 3.677913 \\
\hline 5 & 6 & 0 & 1.473964 & 5.337073 & 2.279040 \\
\hline 6 & 6 & 0 & 3.462459 & -0.343281 & 4.602078 \\
\hline 7 & 6 & 0 & 2.669917 & 5.898803 & 1.489190 \\
\hline 8 & 6 & 0 & 0.130256 & 3.280825 & 3.026832 \\
\hline 9 & 6 & 0 & 1.028278 & 0.490027 & 4.207584 \\
\hline 10 & 6 & 0 & 2.530697 & 0.658963 & 3.894760 \\
\hline 11 & 6 & 0 & 1.362104 & 3.803673 & 2.262106 \\
\hline 12 & 6 & 0 & -4.480886 & 0.962529 & 3.169214 \\
\hline 13 & 6 & 0 & -3.091786 & 1.420691 & 2.700648 \\
\hline 14 & 6 & 0 & 3.335672 & -1.784566 & 4.085669 \\
\hline 15 & 6 & 0 & 4.425772 & -2.725672 & 4.619542 \\
\hline 16 & 6 & 0 & -6.960343 & 0.698053 & 2.564163 \\
\hline 17 & 6 & 0 & 2.595732 & 5.649758 & -0.024305 \\
\hline 18 & 6 & 0 & -5.589783 & 1.248760 & 2.146647 \\
\hline 19 & 6 & 0 & 3.762295 & 6.264539 & -0.811605 \\
\hline 20 & 6 & 0 & 4.279771 & -4.191548 & 4.174128 \\
\hline 21 & 6 & 0 & -8.110643 & 1.029398 & 1.596007 \\
\hline 22 & 6 & 0 & 3.700264 & 6.008892 & -2.328769 \\
\hline 23 & 6 & 0 & 4.344619 & -4.406916 & 2.654800 \\
\hline 24 & 6 & 0 & -7.983031 & 0.465032 & 0.168563 \\
\hline 25 & 6 & 0 & -7.939398 & -1.069079 & 0.092607 \\
\hline 26 & 6 & 0 & 3.913195 & 4.541950 & -2.730473 \\
\hline 27 & 6 & 0 & 4.278797 & -5.885724 & 2.245232 \\
\hline 28 & 6 & 0 & -8.065917 & -1.639768 & -1.332490 \\
\hline 29 & 6 & 0 & 3.765735 & 4.289723 & -4.237890 \\
\hline 30 & 6 & 0 & 4.253035 & -6.128313 & 0.724965 \\
\hline 31 & 6 & 0 & 2.976069 & -5.629956 & 0.032720 \\
\hline 32 & 6 & 0 & -5.550970 & -1.729367 & -1.945072 \\
\hline 33 & 6 & 0 & -6.957027 & -1.244531 & -2.324677 \\
\hline 34 & 6 & 0 & 4.040247 & 2.838285 & -4.670157 \\
\hline 35 & 6 & 0 & 3.092481 & 1.800539 & -4.050851 \\
\hline
\end{tabular}




\begin{tabular}{|c|c|c|c|c|c|}
\hline 36 & 6 & 0 & -3.061133 & -1.771686 & -2.561706 \\
\hline 37 & 6 & 0 & -4.481368 & -1.369400 & -2.985995 \\
\hline 38 & 6 & 0 & 2.908556 & -5.954775 & -1.467328 \\
\hline 39 & 6 & 0 & 1.551495 & -3.932616 & -2.344535 \\
\hline 40 & 6 & 0 & 2.503933 & -0.697935 & -3.814609 \\
\hline 41 & 6 & 0 & 3.341606 & 0.368519 & -4.546084 \\
\hline 42 & 6 & 0 & 0.998073 & -0.676275 & -4.154380 \\
\hline 43 & 6 & 0 & 0.157227 & -3.440569 & -2.782371 \\
\hline 44 & 6 & 0 & 1.618941 & -5.457439 & -2.146577 \\
\hline 45 & 1 & 0 & 1.541249 & 5.677869 & 3.322321 \\
\hline 46 & 1 & 0 & 0.180411 & 3.609582 & 4.077171 \\
\hline 47 & 1 & 0 & 3.283158 & -0.318961 & 5.687137 \\
\hline 48 & 1 & 0 & 0.830564 & 0.812753 & 5.240839 \\
\hline 49 & 1 & 0 & -2.104195 & 1.586796 & 4.634144 \\
\hline 50 & 1 & 0 & 2.737520 & 6.981219 & 1.670166 \\
\hline 51 & 1 & 0 & -4.727541 & 1.443963 & 4.127161 \\
\hline 52 & 1 & 0 & 4.500811 & -0.011240 & 4.457020 \\
\hline 53 & 1 & 0 & 2.835022 & 1.677218 & 4.168454 \\
\hline 54 & 1 & 0 & 3.602310 & 5.467163 & 1.883705 \\
\hline 55 & 1 & 0 & 0.544036 & 5.767426 & 1.878128 \\
\hline 56 & 1 & 0 & 2.282163 & 3.376247 & 2.683581 \\
\hline 57 & 1 & 0 & 4.425058 & -2.688424 & 5.718313 \\
\hline 58 & 1 & 0 & -7.217654 & 1.100659 & 3.554402 \\
\hline 59 & 1 & 0 & -4.448405 & -0.118496 & 3.372637 \\
\hline 60 & 1 & 0 & -0.769767 & 3.756984 & 2.613413 \\
\hline 61 & 1 & 0 & -2.014645 & -0.010342 & 3.918035 \\
\hline 62 & 1 & 0 & 0.755228 & -0.574462 & 4.186765 \\
\hline 63 & 1 & 0 & -3.107471 & 2.506026 & 2.526685 \\
\hline 64 & 1 & 0 & 3.779502 & 7.349027 & -0.632201 \\
\hline 65 & 1 & 0 & 2.349827 & -2.194778 & 4.349082 \\
\hline 66 & 1 & 0 & 5.409921 & -2.348023 & 4.304232 \\
\hline 67 & 1 & 0 & -6.886180 & -0.390634 & 2.695653 \\
\hline 68 & 1 & 0 & 2.701195 & 0.575634 & 2.811836 \\
\hline 69 & 1 & 0 & 1.306287 & 3.457211 & 1.225632 \\
\hline 70 & 1 & 0 & -5.666490 & 2.334405 & 1.983800 \\
\hline 71 & 1 & 0 & 1.648756 & 6.057289 & -0.409955 \\
\hline 72 & 1 & 0 & 4.713913 & 5.877072 & -0.417210 \\
\hline 73 & 1 & 0 & -9.050438 & 0.663041 & 2.033904 \\
\hline 74 & 1 & 0 & 5.068942 & -4.787106 & 4.655045 \\
\hline 75 & 1 & 0 & 3.374741 & -1.763283 & 2.987882 \\
\hline 76 & 1 & 0 & -2.889364 & 0.962782 & 1.722837 \\
\hline 77 & 1 & 0 & 3.326604 & -4.591078 & 4.552171 \\
\hline 78 & 1 & 0 & 2.558152 & 4.570191 & -0.211840 \\
\hline 79 & 1 & 0 & -8.212322 & 2.122386 & 1.531827 \\
\hline 80 & 1 & 0 & -5.295908 & 0.818202 & 1.178902 \\
\hline 81 & 1 & 0 & 2.727071 & 6.352129 & -2.710942 \\
\hline 82 & 1 & 0 & 4.457803 & 6.628641 & -2.829296 \\
\hline 83 & 1 & 0 & -8.763799 & -1.475409 & 0.696833 \\
\hline 84 & 1 & 0 & 5.273111 & -3.964384 & 2.262377 \\
\hline 85 & 1 & 0 & -7.017611 & -1.440719 & 0.559069 \\
\hline 86 & 1 & 0 & 3.522551 & -3.859735 & 2.178050 \\
\hline 87 & 1 & 0 & -8.842765 & 0.822305 & -0.417073 \\
\hline 88 & 1 & 0 & 3.199210 & 3.910251 & -2.189231 \\
\hline 89 & 1 & 0 & 4.914284 & 4.220674 & -2.403507 \\
\hline 90 & 1 & 0 & -7.095035 & 0.889189 & -0.318012 \\
\hline 91 & 1 & 0 & 5.140864 & -6.412861 & 2.677896 \\
\hline 92 & 1 & 0 & 3.386079 & -6.346214 & 2.695005 \\
\hline 93 & 1 & 0 & 4.448773 & 4.957075 & -4.782974 \\
\hline 94 & 1 & 0 & -8.105786 & -2.736804 & -1.268024 \\
\hline 95 & 1 & 0 & 2.749895 & 4.573657 & -4.551216 \\
\hline 96 & 1 & 0 & -9.033813 & -1.325711 & -1.749250 \\
\hline 97 & 1 & 0 & -5.258346 & -1.298917 & -0.976818 \\
\hline 98 & 1 & 0 & 2.883255 & -4.546100 & 0.175778 \\
\hline 99 & 1 & 0 & 5.130149 & -5.650732 & 0.263273 \\
\hline 100 & 1 & 0 & 3.199731 & 1.810117 & -2.957602 \\
\hline 101 & 1 & 0 & -2.857862 & -1.332701 & -1.575034 \\
\hline
\end{tabular}




\begin{tabular}{|c|c|c|c|c|c|}
\hline 102 & 1 & 0 & 2.102565 & -6.078344 & 0.530768 \\
\hline 103 & 1 & 0 & 4.360416 & -7.206113 & 0.536903 \\
\hline 104 & 1 & 0 & -5.568490 & -2.820097 & -1.798699 \\
\hline 105 & 1 & 0 & 5.077966 & 2.574755 & -4.415737 \\
\hline 106 & 1 & 0 & -6.940151 & -0.152690 & -2.448728 \\
\hline 107 & 1 & 0 & 1.847748 & -3.430701 & -1.415897 \\
\hline 108 & 1 & 0 & 2.649764 & -0.563603 & -2.732957 \\
\hline 109 & 1 & 0 & 2.053924 & 2.096731 & -4.255585 \\
\hline 110 & 1 & 0 & -3.021643 & -2.861457 & -2.419344 \\
\hline 111 & 1 & 0 & 3.972330 & 2.776773 & -5.765838 \\
\hline 112 & 1 & 0 & -7.217499 & -1.652231 & -3.311972 \\
\hline 113 & 1 & 0 & -4.503622 & -0.284082 & -3.164865 \\
\hline 114 & 1 & 0 & 0.638351 & 0.360446 & -4.181493 \\
\hline 115 & 1 & 0 & -2.093286 & -0.263191 & -3.778045 \\
\hline 116 & 1 & 0 & -0.596183 & -3.835481 & -2.083217 \\
\hline 117 & 1 & 0 & 3.783432 & -5.525976 & -1.978597 \\
\hline 118 & 1 & 0 & 0.755426 & -5.782799 & -1.546901 \\
\hline 119 & 1 & 0 & 2.302890 & -3.639273 & -3.089032 \\
\hline 120 & 1 & 0 & 4.406742 & 0.131391 & -4.408223 \\
\hline 121 & 1 & 0 & 2.985585 & -7.043514 & -1.596540 \\
\hline 122 & 1 & 0 & 2.910162 & -1.689415 & -4.050993 \\
\hline 123 & 1 & 0 & -4.728315 & -1.840726 & -3.948887 \\
\hline 124 & 1 & 0 & 3.153593 & 0.309696 & -5.628518 \\
\hline 125 & 1 & 0 & -2.096873 & -1.856460 & -4.512903 \\
\hline 126 & 1 & 0 & 0.843204 & -1.070467 & -5.169739 \\
\hline 127 & 1 & 0 & -0.097243 & -3.892918 & -3.753768 \\
\hline 128 & 1 & 0 & 1.506308 & -5.945250 & -3.124889 \\
\hline 129 & 6 & 0 & -0.048893 & -0.738762 & -1.237215 \\
\hline 130 & 6 & 0 & -0.332710 & 0.617698 & -1.093391 \\
\hline 131 & 9 & 0 & -0.641062 & 1.366515 & -2.183120 \\
\hline 132 & 6 & 0 & -0.348831 & 1.255978 & 0.144219 \\
\hline 133 & 9 & 0 & -0.692301 & 2.568020 & 0.159319 \\
\hline 134 & 6 & 0 & -0.047892 & 0.593252 & 1.331413 \\
\hline 135 & 6 & 0 & 0.243997 & -1.421428 & -0.041264 \\
\hline 136 & 6 & 0 & 0.255397 & -0.776162 & 1.195323 \\
\hline 137 & 1 & 0 & 0.457060 & -2.485362 & -0.072215 \\
\hline 138 & 1 & 0 & 0.495381 & -1.359301 & 2.080012 \\
\hline
\end{tabular}

Dipole moment (field-independent basis, Debye):
$\mathrm{X}=$
0.4532
$\mathrm{Y}=$
$-1.7487 \quad Z=$
0.8251 Tot $=$
1.9860 
Table S3. Optimized Structural Coordinate and its Total Energy for C18PhF2 at B3LYP/6-31G(d) level

$$
\text { total energy: } \mathrm{HF}=-3131.467809 \text { hartree }(\mathrm{NImag}=0)
$$

\begin{tabular}{|c|c|c|c|c|c|}
\hline \multirow{2}{*}{$\begin{array}{c}\text { Center } \\
\text { Number }\end{array}$} & \multirow{2}{*}{$\begin{array}{l}\text { Atomic } \\
\text { Number }\end{array}$} & \multirow{2}{*}{$\begin{array}{l}\text { Atomic } \\
\text { Type }\end{array}$} & \multicolumn{3}{|c|}{ Coordinates (Angstroms) } \\
\hline & & & $\mathrm{X}$ & $\mathrm{Y}$ & \\
\hline 1 & 14 & 0 & -1.931116 & -2.880491 & -0.237759 \\
\hline 2 & 14 & 0 & 0.803918 & 3.074608 & -1.665665 \\
\hline 3 & 6 & 0 & -3.750814 & -2.862403 & -0.796845 \\
\hline 4 & 6 & 0 & 4.196252 & 5.304519 & 3.337478 \\
\hline 5 & 6 & 0 & 2.750127 & 5.632943 & 1.236315 \\
\hline 6 & 6 & 0 & 2.296307 & 5.075056 & -0.126821 \\
\hline 7 & 6 & 0 & 5.031458 & 4.328178 & 4.185832 \\
\hline 8 & 6 & 0 & 3.655717 & 4.688532 & 2.038638 \\
\hline 9 & 6 & 0 & 6.636613 & 0.918019 & -4.523847 \\
\hline 10 & 6 & 0 & 8.569153 & -0.140276 & -3.157573 \\
\hline 11 & 6 & 0 & 4.427066 & 1.948254 & -3.723097 \\
\hline 12 & 6 & 0 & 7.649467 & -0.231119 & -4.385376 \\
\hline 13 & 6 & 0 & 2.169956 & 2.921294 & -2.977194 \\
\hline 14 & 6 & 0 & 5.552715 & 0.946343 & -3.434917 \\
\hline 15 & 6 & 0 & 4.208504 & 3.272961 & 4.942867 \\
\hline 16 & 6 & 0 & 1.497398 & 3.760363 & -0.031905 \\
\hline 17 & 6 & 0 & 9.521233 & -1.342499 & -3.003974 \\
\hline 18 & 6 & 0 & -0.590858 & 4.172584 & -2.350340 \\
\hline 19 & 6 & 0 & 3.351678 & 1.997760 & -2.627305 \\
\hline 20 & 6 & 0 & 5.085606 & 2.207618 & 5.616625 \\
\hline 21 & 6 & 0 & -3.064343 & 4.897993 & -2.184760 \\
\hline 22 & 6 & 0 & -1.878914 & 4.194126 & -1.506049 \\
\hline 23 & 6 & 0 & -5.623530 & 5.218724 & -2.161865 \\
\hline 24 & 6 & 0 & 8.838600 & -2.658860 & -2.585316 \\
\hline 25 & 6 & 0 & -4.382995 & 4.686585 & -1.428691 \\
\hline 26 & 6 & 0 & 4.343408 & 1.241117 & 6.554276 \\
\hline 27 & 6 & 0 & -6.961761 & 4.789376 & -1.531242 \\
\hline 28 & 6 & 0 & 8.402626 & -2.696170 & -1.110616 \\
\hline 29 & 6 & 0 & 3.216416 & 0.425828 & 5.900675 \\
\hline 30 & 6 & 0 & -7.216220 & 3.277070 & -1.606710 \\
\hline 31 & 6 & 0 & 7.476058 & -3.872546 & -0.753642 \\
\hline 32 & 6 & 0 & 6.038058 & -3.700516 & -1.264687 \\
\hline 33 & 6 & 0 & 2.709497 & -0.708978 & 6.804998 \\
\hline 34 & 6 & 0 & -8.569095 & 2.819471 & -1.046721 \\
\hline 35 & 6 & 0 & 0.156120 & -0.699285 & 6.436131 \\
\hline 36 & 6 & 0 & 1.478859 & -1.464629 & 6.270813 \\
\hline 37 & 6 & 0 & 5.098124 & -4.855284 & -0.895643 \\
\hline 38 & 6 & 0 & -8.694759 & 1.290055 & -1.035668 \\
\hline 39 & 6 & 0 & 3.651592 & -4.614964 & -1.348522 \\
\hline 40 & 6 & 0 & -1.093882 & -1.463738 & 5.965615 \\
\hline 41 & 6 & 0 & -1.231658 & -1.595357 & 4.441660 \\
\hline 42 & 6 & 0 & -10.040278 & 0.761390 & -0.521583 \\
\hline 43 & 6 & 0 & 0.508626 & -4.404964 & -0.707357 \\
\hline 44 & 6 & 0 & -2.787154 & -2.281158 & 2.492829 \\
\hline 45 & 6 & 0 & 2.694310 & -5.761095 & -0.993442 \\
\hline 46 & 6 & 0 & -2.523216 & -2.307205 & 4.009935 \\
\hline 47 & 6 & 0 & -1.822485 & -3.140011 & 1.647097 \\
\hline 48 & 6 & 0 & -0.964828 & -4.253071 & -1.131848 \\
\hline 49 & 6 & 0 & 1.239084 & -5.540711 & -1.442061 \\
\hline 50 & 6 & 0 & -10.109744 & -0.774826 & -0.443502 \\
\hline 51 & 6 & 0 & -9.307922 & -1.382308 & 0.719485 \\
\hline 52 & 6 & 0 & -4.476215 & -4.216950 & -0.656186 \\
\hline 53 & 6 & 0 & -6.863102 & -3.438907 & -0.013968 \\
\hline 54 & 6 & 0 & -5.975040 & -4.196064 & -1.012450 \\
\hline 55 & 6 & 0 & -9.286201 & -2.922255 & 0.729462 \\
\hline 56 & 6 & 0 & -8.363901 & -3.580976 & -0.314173 \\
\hline 57 & 1 & 0 & 4.810503 & 6.179761 & 3.083601 \\
\hline
\end{tabular}




\begin{tabular}{|c|c|c|c|c|c|}
\hline 58 & 1 & 0 & 3.278121 & 6.583316 & 1.070380 \\
\hline 59 & 1 & 0 & 5.624001 & 4.896401 & 4.916361 \\
\hline 60 & 1 & 0 & 1.693654 & 5.842889 & -0.631620 \\
\hline 61 & 1 & 0 & 3.181555 & 4.920817 & -0.760360 \\
\hline 62 & 1 & 0 & 3.359001 & 5.687011 & 3.940507 \\
\hline 63 & 1 & 0 & 7.168287 & 1.881221 & -4.536655 \\
\hline 64 & 1 & 0 & 1.862275 & 5.878584 & 1.838092 \\
\hline 65 & 1 & 0 & 4.506212 & 4.380652 & 1.410890 \\
\hline 66 & 1 & 0 & 9.165952 & 0.779836 & -3.234810 \\
\hline 67 & 1 & 0 & 4.852927 & 2.953860 & -3.858148 \\
\hline 68 & 1 & 0 & 5.761044 & 3.823348 & 3.534653 \\
\hline 69 & 1 & 0 & 2.544084 & 3.934250 & -3.191609 \\
\hline 70 & 1 & 0 & -0.209841 & 5.195263 & -2.489690 \\
\hline 71 & 1 & 0 & 6.145568 & 0.830801 & -5.503662 \\
\hline 72 & 1 & 0 & 8.276596 & -0.265154 & -5.287970 \\
\hline 73 & 1 & 0 & 3.104324 & 3.769672 & 2.274044 \\
\hline 74 & 1 & 0 & 10.297447 & -1.096277 & -2.265306 \\
\hline 75 & 1 & 0 & 3.951614 & 1.689978 & -4.680908 \\
\hline 76 & 1 & 0 & 10.047979 & -1.496361 & -3.956568 \\
\hline 77 & 1 & 0 & 6.003951 & 1.179798 & -2.460523 \\
\hline 78 & 1 & 0 & -2.853573 & 5.971494 & -2.298282 \\
\hline 79 & 1 & 0 & 7.967699 & -0.030986 & -2.245873 \\
\hline 80 & 1 & 0 & 5.877196 & 2.709710 & 6.191621 \\
\hline 81 & 1 & 0 & 3.817661 & 2.320700 & -1.684657 \\
\hline 82 & 1 & 0 & 3.591941 & 3.777839 & 5.702070 \\
\hline 83 & 1 & 0 & 1.701620 & 2.574775 & -3.909045 \\
\hline 84 & 1 & 0 & -0.827860 & 3.802582 & -3.358100 \\
\hline 85 & 1 & 0 & 0.660250 & 3.895805 & 0.670018 \\
\hline 86 & 1 & 0 & 2.133175 & 2.983630 & 0.413533 \\
\hline 87 & 1 & 0 & -5.576490 & 6.315793 & -2.206258 \\
\hline 88 & 1 & 0 & 3.505163 & 2.784069 & 4.256229 \\
\hline 89 & 1 & 0 & -3.173169 & 4.499018 & -3.204154 \\
\hline 90 & 1 & 0 & 5.601740 & 1.628244 & 4.836453 \\
\hline 91 & 1 & 0 & 7.094808 & -1.179690 & -4.367967 \\
\hline 92 & 1 & 0 & -1.683395 & 4.665328 & -0.531777 \\
\hline 93 & 1 & 0 & 5.117633 & -0.059672 & -3.334888 \\
\hline 94 & 1 & 0 & 2.978325 & 0.980143 & -2.445128 \\
\hline 95 & 1 & 0 & -4.321102 & 5.148010 & -0.431699 \\
\hline 96 & 1 & 0 & -5.599464 & 4.871480 & -3.205761 \\
\hline 97 & 1 & 0 & 3.928753 & 1.804426 & 7.403511 \\
\hline 98 & 1 & 0 & 7.892744 & -1.759516 & -0.844276 \\
\hline 99 & 1 & 0 & -7.780970 & 5.317326 & -2.039707 \\
\hline 100 & 1 & 0 & 9.528836 & -3.495063 & -2.764381 \\
\hline 101 & 1 & 0 & -2.179546 & 3.161103 & -1.282936 \\
\hline 102 & 1 & 0 & 7.975021 & -2.843493 & -3.236767 \\
\hline 103 & 1 & 0 & 9.304006 & -2.736097 & -0.483072 \\
\hline 104 & 1 & 0 & -6.995479 & 5.114557 & -0.480610 \\
\hline 105 & 1 & 0 & 5.079023 & 0.546361 & 6.985001 \\
\hline 106 & 1 & 0 & -4.497256 & 3.608739 & -1.254453 \\
\hline 107 & 1 & 0 & 2.386394 & 1.093363 & 5.634400 \\
\hline 108 & 1 & 0 & 3.575918 & -0.007241 & 4.954676 \\
\hline 109 & 1 & 0 & -7.133959 & 2.952245 & -2.655043 \\
\hline 110 & 1 & 0 & 5.630076 & -2.762829 & -0.857833 \\
\hline 111 & 1 & 0 & 6.039111 & -3.579118 & -2.356988 \\
\hline 112 & 1 & 0 & -6.424000 & 2.743442 & -1.064959 \\
\hline 113 & 1 & 0 & 7.447140 & -3.987862 & 0.339106 \\
\hline 114 & 1 & 0 & 7.900348 & -4.808762 & -1.146386 \\
\hline 115 & 1 & 0 & 2.475738 & -0.304988 & 7.801678 \\
\hline 116 & 1 & 0 & -9.386121 & 3.258359 & -1.638286 \\
\hline 117 & 1 & 0 & -8.693028 & 3.202443 & -0.022775 \\
\hline 118 & 1 & 0 & 0.206977 & 0.263194 & 5.906986 \\
\hline 119 & 1 & 0 & 3.532405 & -1.421669 & 6.955978 \\
\hline 120 & 1 & 0 & 0.036328 & -0.452288 & 7.500730 \\
\hline 121 & 1 & 0 & 3.294719 & -3.677485 & -0.900384 \\
\hline 122 & 1 & 0 & 1.642968 & -1.721212 & 5.214598 \\
\hline 123 & 1 & 0 & 3.633133 & -4.456196 & -2.437399 \\
\hline
\end{tabular}




\begin{tabular}{|c|c|c|c|c|c|}
\hline 124 & 1 & 0 & -1.215585 & -0.587081 & 3.999243 \\
\hline 125 & 1 & 0 & 5.116595 & -5.006784 & 0.194103 \\
\hline 126 & 1 & 0 & 5.472630 & -5.790184 & -1.338470 \\
\hline 127 & 1 & 0 & -8.530002 & 0.907280 & -2.054211 \\
\hline 128 & 1 & 0 & -7.877546 & 0.880121 & -0.425829 \\
\hline 129 & 1 & 0 & -1.986246 & -0.949422 & 6.349788 \\
\hline 130 & 1 & 0 & 1.389705 & -2.421606 & 6.804639 \\
\hline 131 & 1 & 0 & 1.033413 & -3.455784 & -0.876279 \\
\hline 132 & 1 & 0 & -2.757966 & -1.234282 & 2.157760 \\
\hline 133 & 1 & 0 & -10.839394 & 1.126273 & -1.182036 \\
\hline 134 & 1 & 0 & -0.359830 & -2.123700 & 4.033888 \\
\hline 135 & 1 & 0 & -10.249686 & 1.186135 & 0.471771 \\
\hline 136 & 1 & 0 & -0.782603 & -2.963521 & 1.954595 \\
\hline 137 & 1 & 0 & -1.101629 & -2.464422 & 6.423196 \\
\hline 138 & 1 & 0 & -4.270945 & -2.089761 & -0.213830 \\
\hline 139 & 1 & 0 & 1.215873 & -5.345299 & -2.524555 \\
\hline 140 & 1 & 0 & -3.793415 & -2.528361 & -1.842154 \\
\hline 141 & 1 & 0 & -3.371900 & -1.826629 & 4.518166 \\
\hline 142 & 1 & 0 & -1.019935 & -4.063807 & -2.213543 \\
\hline 143 & 1 & 0 & 0.565962 & -4.594929 & 0.374658 \\
\hline 144 & 1 & 0 & 2.715236 & -5.929033 & 0.094084 \\
\hline 145 & 1 & 0 & 3.069433 & -6.688543 & -1.449261 \\
\hline 146 & 1 & 0 & -3.814419 & -2.621886 & 2.308172 \\
\hline 147 & 1 & 0 & -8.278287 & -1.001846 & 0.709370 \\
\hline 148 & 1 & 0 & -9.765375 & -1.194047 & -1.399827 \\
\hline 149 & 1 & 0 & -2.506105 & -3.348636 & 4.364000 \\
\hline 150 & 1 & 0 & -6.582948 & -2.377524 & 0.002084 \\
\hline 151 & 1 & 0 & -2.005468 & -4.203826 & 1.859838 \\
\hline 152 & 1 & 0 & 0.676256 & -6.472960 & -1.290656 \\
\hline 153 & 1 & 0 & -1.491908 & -5.205050 & -0.967637 \\
\hline 154 & 1 & 0 & -11.158703 & -1.085945 & -0.337963 \\
\hline 155 & 1 & 0 & -9.747762 & -1.026622 & 1.662045 \\
\hline 156 & 1 & 0 & -6.104018 & -3.769837 & -2.018298 \\
\hline 157 & 1 & 0 & -4.369945 & -4.596968 & 0.371300 \\
\hline 158 & 1 & 0 & -3.979159 & -4.954174 & -1.300670 \\
\hline 159 & 1 & 0 & -6.667294 & -3.822538 & 0.999441 \\
\hline 160 & 1 & 0 & -8.577360 & -3.178522 & -1.314651 \\
\hline 161 & 1 & 0 & -8.982737 & -3.270907 & 1.726951 \\
\hline 162 & 1 & 0 & -10.312522 & -3.288130 & 0.582332 \\
\hline 163 & 1 & 0 & -6.330221 & -5.235189 & -1.074671 \\
\hline 164 & 1 & 0 & -8.606485 & -4.651959 & -0.365736 \\
\hline 165 & 6 & 0 & -1.126960 & -1.192670 & -0.628445 \\
\hline 166 & 6 & 0 & -1.117363 & -0.664917 & -1.918537 \\
\hline 167 & 9 & 0 & -1.686940 & -1.358479 & -2.936626 \\
\hline 168 & 6 & 0 & -0.536445 & 0.565163 & -2.218136 \\
\hline 169 & 9 & 0 & -0.587746 & 0.992129 & -3.505065 \\
\hline 170 & 6 & 0 & 0.076113 & 1.358291 & -1.249614 \\
\hline 171 & 6 & 0 & -0.498244 & -0.404393 & 0.353568 \\
\hline 172 & 6 & 0 & 0.080875 & 0.828508 & 0.054123 \\
\hline 173 & 1 & 0 & -0.461501 & -0.757862 & 1.379237 \\
\hline 174 & 1 & 0 & 0.541911 & 1.393528 & 0.858563 \\
\hline
\end{tabular}

Dipole moment (field-independent basis, Debye):

$$
\mathrm{X}=0.4650 \quad \mathrm{Y}=0.2061 \quad \mathrm{Z}=1.9047 \text { Tot }=
$$

